九州大学学術情報リポジトリ

Kyushu University Institutional Repository

\title{
A Revision 0f The Asiatic Buxus
}

Hatusima, Sumihiko

Forestry Laboratory, Department of Agriculture, Kyusyu Imperial University

https://doi.org/10.5109/22593

出版情報：九州大学大学院農学研究院紀要. 6 (6)，pp. 261-342，1942-02. Kyushu Imperial University バージョン：

権利関係 : 
Journal of the Department of Agriculture, Kyügȳ̄ Imperial University, Vol. 6, No. 6, February 15th, 1942

\title{
A REVISION OF THE ASIATIC BUXUS
}

\author{
Sumihiko Hatusma
}

\section{Introduction}

Since the BAILLoN's classical work "Monographie des Buxacees et des Stylocérées" (1859) was published, a number of species of Buxus has been described by many systematists, such as H. F. Hance, T. Makino, C. K. Schneider, H. Léveillé, A. Rehder and E. H. Wilson, W. W. Smith, F. Gagnepain, H. N. Ridley, and E. D. MERRILL. Yet no revision or synopsis of all the species of Buxus occurring in Asia has ever been published.

The present work is a revision of all the species of Buxus occurring in Asia, excluding those from Syria. The specific description and figures are mostly original and drawn from living or dried specimens, or both.

In the nomenclature the writer adopted the International Rule of Botanical Nomenclature (Cambridge).

The present work has been carried out chiefly based upon the materials preserved in the following herbaria:

1. Herbarium of the Arnold Arboretum (abbreviated as HA in the citation of specimens).

2. Herbarium of the Shanghai Science Institute (abbreviated as HSa).

3. Herbarium of the Faculty of Forestry, Kyūsyū Imperial University (no special sign is given after the citation of specimens).

4. Herbarium of the Botanic Gardens, Singapore (abbreviated as HS).

5. Herbarium of the Forest Experiment Station, Taiwan (abbreviated as $\mathrm{HT}$ ). 
My hearty thanks are due to Dr. R. KanehiRA, Professor at Kyūsy $\bar{u}$ Imperial University, for his useful advice 'and encouragement. Through his help I was able to obtain the loan of a great number of specimens of the Asiatic Buxus from the Herbarium of the Anold Arboretum.

I wish to express my appreciation to Dr. E. D. MERRILL, Director of the Arnold Arboretum who kindly loaned a great number of specimens preserved in the herbarium and enabled me to accomplish this work.

I am also very much obligated to Mr. E. D. HolTtum, Director of the Botanic Gardens at Singpore, to Mr. Migo, taxonomist of the Department of Biology, Shanghai Science Institute, and to Dr. F. SEKI, Director of the Forest Experiment Station, Taiwan for the loan of speciemens. Finally I must say that I am under obligation to many botanists and collectors of the different parts of Japan who sent many valuable specimens to me and have thus enabled to obtain many interesting data regarding the distribution of the Buxus in Japan.

\section{Buxus Linnaeus}

Buxus Linnaeus (Syst. Nat. 9, 1735; Gen. Pl. 284, 1737); Sp. Pl. ed. 1: 983 (1753); ed. 2: 1394 (1762).-ENDLICHER, Gen. P1. no. 5869 (1836-40).-Baillon, Monogr. Bux. et Styl. 58 (1859), pro. parte.-Mueller Arg. in De Candolle, Prod. 16: 13 (1869), pro parte.-Bentham et Hooker, Gen. Pl. 3: 266 (1880), pro parte.van TIEghem in Ann. Sci. Nat. 5: 289 (1897), pro parte.-PAX in Engler u. Prantl, Nat. Pfl.-fam. 3 (5): 130 (1890), pro parte.Hayata in Journ. Coll. Sci. Imp. Tokyo 20. art. 3:82 (1904).HutChinson in Kew Bull. 52 (1919):-GAGNEPAIN in LeComte, Fl. Gén. Indo-Chine 5: 660 (1927) (sub Euphorbiaceae).

Frutices vel arborescentes sempervirentes, ramulis tetragonis vel tereti-tetragonis. Folia opposita breviter petiolata integerrima coriacea vel tenuiter coriacea, pennivenia. Inflorescentiae axillares spicatae vel racemosae breves solitares, bracteae saepe numerosae oppositae, segmentis perianthii similes nisi minores; flores $\hat{\delta}$ laterales et basilares, sub quaque bractea solitariis sessilis. Flores $\hat{o}:$ Sepala 4, opposita, imbricata. Stamina 4, sepalis opposita, filamentis liberis exsertis, antherae juxta basin dorsifixae, oblongae, demum recurvae, 
loculis introrsum adnatis parallelis longitudinaliter dehiscentibus. Ovarii rudimentum truncatum tetragonum saepissime 4-lobum sepalis fere aequans vel breviore glabrum. Flos $ᄋ$ : Sepala 6, valde imbricata, exteriora paullo minora. Ovarium 3-loculare, styli breves, crassi, inter se distantes vel rarius basi contigui, ovula in loculis gemina, ab apice pendula, ab angulo interno distantia, raphe dorsali, microphyle axin spectante. Capsula ovoidea, stylis persistentibus 3-cornuta, loculicide dehiscens, valvis indivisis stylis fissis 2-cornutis; pericarpio indurato, endocarpio soluto cartilagineo. Semina oblonga, 3-quetra, strophiola parva, testa crustacea nigra nitida; albumen subcarnosum, cotyledones oblongae, radicula vix latiores.

Species ad 29, Asiae et Europae incolae.

Criteria of classification:

In determining each species and varieties of the Buxus, the following characters are used in this work.

The branchlets. The box has generally quadrangular branchlets, while in some the branchlets are nearly round (B. Henryi). The colour of the branchlets are generally greenish, but in B. papillosa glaucous.

The leaf. The shape, size and texture of the leaves are considerably variable even in the same species according to the conditions under which it grows, and therefore of little importance in this genus. The species of the Sect. Eugeniobuxus have generally larger trinerved acuminate leaves with midribs distinctly impressed above when dried, while in the species of the Sect. Eubuxus leaves are generally smaller and penninerved, obtuse or shortly emarginate rarely shortly acuminate at the apex, and the midribs are prominently elevated above, though in a few species they are flattened or slightly impressed (B. papillosa, B. rupicola). In some species the midribs are distinctly elevated beneath when dried and they sometimes serve as a distinguishing character of the species (B. Bodinieri, B. Harlandii, B. stenophylla, B. austroyunnanensis, $B$. Myrica, $B$. hainanensis and $B$. Wallichiana), while $B$. rugulos $a$ and its subspecies and variety, $B$. ichangensis, $B$. papillos $a, B$. cephalanthera and $B$. microphylla have slightly elevated midribs beneath which, in some species, are densely covered with white cystoliths and looks white in colour (B. microphylla, together with its subspecies and variety). 
The characters of the lateral nerves are also important for taxonomic purpose. In some species the lateral nerves are very distinct and delicately reticulated on both surfaces when dried $(B$. austro-yunnanensis, B. Myrica, B. Harlandii and B. stenophylla), while in some species they are scarcely visible on both surfaces (B. papillosa, B. rupicola, B. mollicula and B. rugulosa, together with its subspecies and variety).

The leaves are generally green, glabrous and shining above, bjt occasionally glaucous and dull (B. papillosa, B. mollicula, $B$. rupicola and $B$. rugulos $a$ subsp. rupicola), in some cases densely papillous beneath (B. papillosa, B. rigulosa subsp. rupicola); in some species the leaves are more or less densely covered with soft adpressed hairs on both surfaces which soon disappear $(B$. rugulosa subsp. rupicola, $B$. mollicula var. glabra) or are more or less retained in the second year (B. mollicula).

The inflorescence. The inflorescence is of great importance; it may be small raceme or spike. The following species have sessile male flowers; $B$. microphylla with its subspecies, B. stenophylla, $B$. hebecarpa?, B. mollicula?, B. Wallichiana, B. papillosa. The length of the floral axes affords a useful distinctive character, though occasionally they may not be constant individually. In the species of the Sect. Egeniobuxus the racemes are generaily loose and longer than the petioles, and the floral axes are glabrous, while in the species of the Sect. Eubuxus they are relatively shorter and denser than those of the Sect. Eugeniobuxus, and the floral axes are more or less puberulent, though $B$. austro-yunnanensis, $B$. Myrica, $B$. megistophylla, etc. have loose elongate racemes. The number of the bracts, hairiness, colour, texture, and nature of margine-scarious or not-of the bracts and sepals are also very important for taxonomic purposes. The species of the Sect. Eugeniobuxus have usually thicker and narrower bracts and sepals than those of the species belonging to the Sect. Eubuxus. In B. papillosa, $B$. Wallichiana and B. Henryi, they are very thin and scarious. The male flowers are relatively uniform in shape, though they vary considerably in their size; B. Wallichiana, B. Henryi, B. rugulosa, $B$. microphylla and $B$. liukiuensis have relatively large flowers. In the species of the Sect. Eugeniobuxus the male flowers have usually longer pedicels often measuring about $6 \mathrm{~mm}$. long, than those of the species of the Sect. Eubuxus, such as in B. Henryi $(2.5 \mathrm{~mm}$.), $B$. 
hainanensis (2.5 mm.), B. liukiuensis var. longipedicellata $(1.7 \mathrm{~mm}$.$) ,$ B. Myrica (1-1.5 mm.), B. austro-yunnanensis, B. rugulosa, together with its subspecies and variety, $B$. ichangensis, $B$. Bodinieri and B. Harlandii (about $1 \mathrm{~mm}$. long respectively).

The height and nature of the rudimental ovaries are also very important for taxonomic purposes, though they slightly elongate as the flowers develope. The following 19 species have rudimental ovaries scarcely half as long as or not more than one-third as long as the sepal.

All species of the Sect. Eugeniobuxuis, B. rupicola, B. malayana, B. latistyla, B. Myrica, B. hainanensis, B. liukiuensis, B. megistophylla, $B$. austro-yunnanensis, $B$. Henryi, $B$. mollicula, $B$. papillos $a, B$. rugulosa, together with its subspecies and variety, $B$. Wallichiana.

In $B$. Harlandii, B. stenophylla and $B$. cephalanthera the rudimental ovaries are about two-third as long as the sepal, while in $B$. microphylla, $B$. Bodinieri and $B$. ichangensis they are nearly equal as long as the sepals or slightly longer. Most species have rudimental ovaries suddenly dilated at the top, but in some species, namely $B$. papillos $a$ and $B$. Henryi, the tops are scarcely dilated.

The nature of the ovary, especially the shape of the styles and stigmas on which most previous authors have not laid stress except the work of GaGNEPAIN (LeComTE, Fl. Gén. Indo-Chine) is of most important.

The following species have complanate styles usually gradually tapering and recurving toward the apex and more or less longer than the ovary, the stigmas of which are narrowly obcordate to linear-obcordate usually decurrent to the nearly two-third as long as or almost to the base of the style: all species of the Sect. Engeniobubus, $B$. austro-yunnanensis (stigma is very short), $B$. Myrica, B. papillosa, B. Henryi, B. megistophylla, B. liukiuensis, B. malayana, B. latistyla, B. rupicola, and B. stenophylla.

The following species have thicker scarcely complanate styles usually more or less shorter than the ovary and often dilating toward the apex, the stigmas of which are obcordate and decurrent scarcely as half to" two-third as long as the style: $B$. microphylla, together with its subspecies, B. rugulosa together with 
its subspecies and variety, $B$. Wallichiana, B. hebecarpa, B. Bodinieri, $B$. .cephalanthera, $B$. ichangensis.

The fruit. The fruits of the Buxus are reasonably uniform in shape, though they are considerably variable in their size. Small shrubs, such as $B$. Harlandii, B. ichangensis, B. cephalanthera, $B$. Bodinieri, B. rugulosa subsp. prostrata, etc. have relatively small fruits. Most species have glabrous shining capsules, but in some species they are dull (B. rupicola, B. liukiuensis, all species of the Sect. Eugeniobuxus), and occasionally densely pubescent ( $B$. hebecarpa, B. rugulosa subsp. rupicola, B. cephalanthera; in the two of the latter, however, the hairs soon disappear).

The angle formed by the horns of the capsules also serves as a useful distinctive character in some species, though the length of it is very variable in an individiual.

Geographical distribution.

Twenty-six species of Buxus are recognized in this work, of which 20 species are distributed among Sect. Eubuxus and remaining 6 among Sect. Eugeniobuxus. A few species have wide distribution but the greater number is of very local. The most widely distributed species are B. microphylla var. japonica and B. microphylla subsp. sinica. The first-named is confined to Japan proper ranging from about lat. $30^{\circ} \mathrm{N}$ in the island of Yakusima in Kyūsyū, northward to the Province of Mutu, northern part of Japan, at about lat. $38^{\circ} 5^{\prime} \mathrm{N}$. The second-named is most widely distributed throughout the central and northern provinces of China, extending from southern Shensi, Kansu and Shantung southward to northern Kiangsi, Checkiang, Hupeh and westward to Szech'uan. It reappears on the mountains of Formosa and also in a closely related form, known as variety insularis, in the warmer parts of Korea.

$B$. Bodinieri is also widely distributed throughout the provinces south of the river of Yangtsekiang, occurring in Fukien, Hupeh, Kweichow, Szech'uan, Kwangsi, Kwangtung and southern Yunnan bordering to Burma.

B. rugulosa, together with its variety intermedia and subspiecies rupicola is a most common box to be found on the alpine regions of north-western Yunnan and western Szech'uan, and its subspecies prostrata also extends westward from Yunnan through Tibet to the north-western Himalaya. 
B. rupicola recorded from the Malay Peninsula is a southernmost representative of Buxus to be found in Asia.

The remaining species are very restricted in their distribution. The majority of the species is Chinese; in west-central China the genus reaches its greatest development and spreads there to northern and eastern China.

Judging from its distribution, which can be better appreciated by a glance at the accompanying sketch map and table, it is almost certain that the central home of Buxus lies in these area.

The distribution of the species of the Sect. Eugeniobuxus is of remarkable interest. Of six known species which are all closely related to each other, four are peculiar to the Philippines where it reaches its greatest development, one from Cochin-China, one from the Malay Peninsula, thus all species are confined to the tropical Asia south of about lat. $15^{\circ} \mathrm{N}$.

Clavis sectionum

Folia apice acuminata vel acuta, trinervia, costa media in sicco supra valde impressa. ............................ Sect. Eugeniobuxus Folia apice emarginata vel obtuse acuta nunquam acuminata, pennivenia, costa media supra in sicco valde elevata rarius plana. Sect. Eubuxus

Sect. Eugeniobuxus Hatusima, sect. nov.

Frutex vel arbor parva glaberrima. ramuli tetragoni angulati. Folia plerumque magna, coriacea vel tenuiter coriacea, ovato-lanceolata vel ovato-oblonga vel lanceolata, apice acuminata vel breviter acuminata vel acuta, nervis lateralibus saepe utraque facie distinctis rarius obsoletis prope ad marginem in nervo antemarginale coalitis, supra costa media valde impressa subtus prominente elevata. Racemi axillares laxiflores, rhachi elongata glabra; flores ô longe pedicellati, ovarii rudimentum sessile sepalis multo breviore; flos 우 terminalis, ovarium stylis complanatis apicem versus sensim angustatis saepe recurvatis plus minusve longiore glabrum, stigmata anguste oblanceolata fere usque ad basim stylum decurrentia. Capsula opaca glabra.

Typus; Buxus Rolfei VIDAL. Species ad 6, Asiae tropicae incolae. 
The species of this section are well characterized by its quite glabrous nature, its generally acuminate large trinerved leaves with the midrib distinctly impressed above when dried, its elongated racemes bearing long-pedicelled male flowers with rudimental ovaries distinctly shorter than the sepal, and its dull scabrous capsules. In regard to the leaves, species of this section bear a strong resemblance to those of the certain species of Eugenia, from which I have given a name of Eugeniobuxus above.

Of the six species of this -section, one is found in CochinChina; one occurs in the Malay Peninsula. The remaining four species are peculiar to the Philippines. From this analysis it would appear that the Philippines are the headquarter of this section.

Conspectus specierum sectionis Eugeniobuxi.

1) Folia oblonga, crassissime coriacea, circ. $10 \mathrm{~cm}$. longa, $3-5 \mathrm{~cm}$. lata, margine valde revoluta, utraque facie nitidula, nervis lateralibus utraque facie obsoletis; pedunculi fructiferi vix ultra $5 \mathrm{~mm}$. longi............................... 1. B. pachyphylla

1) Folia coriacea vel tenuiter coriacea, margine leviter revoluta; pedunculi fructiferi plerumque ultra $1 \mathrm{~cm}$. longi.

2) Folia lanceolata $2.5-5 \mathrm{~cm}$. longa. 2. B. rivularis

2) Folia plerumque ultra $5 \mathrm{~cm}$. longa.

3) Capsula obovoidea stylis persistentibus divaricatis vix recurvatis circ. duplo longiora; folia tenuiter coriacea, supra nitida, $10-17 \mathrm{~cm}$. longa. ................. 3. B. Holttumiana.

3) Capsula globoso-ovoidea vel ovoidea, styli persistentes apice valde recurvati.

4) Capsula globoso-ovoidea, stylis persistentibus paullo longiora; folia lanceolata vel ovato-lanceolata $5-10 \mathrm{~cm}$. longa supra nitida, nervis, lateralibus supra distinctis. 4. B. cochinchinensis

4) Capsula ovoidea, stylis persistentibus $3-4$ plo longiora.

5) Folia lanceolata vel late lanceolata, supra nitidula, tenuiter coriacea, $10-15 \mathrm{~cm}$. longa, $1.5-8 \mathrm{~cm}$. lata, nervis lateralibus supra distinctis......... 5. B. Rolfei.

5) Folia lanceolata vel late lanceolata, coriacea, supra nitida, 5-6 cm. longa, $1.5-2 \mathrm{~cm}$. lata. ... 6. B. Loheri. 
1. Buxus pachyphylla Merrill in 'Philip. Journ. Sci. 9: Bot. 310 (1914); Enum. Philip. Fl. Pl. 2: 464 (1923).

Arbor parva glabra, ramuli hornotini tetragoni longitudinaliter 2-sulcati graciles, vetustiores teretes pallide olivacei. Folia breviter petiolata, crassissime coriacea, oblonga, 9-11 cm. longa, 3-5.5 cm. lata, apice acuminata, basi acuta vel acuminata, margine valde revoluta, utrinque nitida, subtus pallidiora, nervis lateralibus tenuibus utrinsecus circ. 30 a costa egresis, supra saepe obsoletis cum nervis secundariis vel reticulatis conjunctis. Peduculi axillares solitarii circ. $5 \mathrm{~mm}$. longi. .Capsula ovoidea circ. $1 \mathrm{~cm}$. longa, glabra, saepe in sicco glauco-purpurascens.

Philippine. Luzon: Tayabas: Mt. Cadig near Guinayangan, March 9, 1913 (EsCRIToR; B. S. no. 20828, type).

Of this species 1 have not yet seen any specimen, but it is very closely related to Buxus Rolfei VIDAL, from which it differs, according to MerRILL, in its very thickly coriaceous leaves, and in its shorter peduncles.

2. Buxus rivularis Merrill in Philip. Journ. Sci. 9: Bot. 309 (1914); Enum. Philip. Fl. Pl. 2: 465 (1923).

Frutex glaber circ. $1 \mathrm{~m}$. altus, ramuli hornotini tetragoni, vetustiores subteretes cinerascentes graciles. Folia lanceolata, coriacea vel subcoriacea, breviter petiolata, $2.5-5 \mathrm{~cm}$. longa $0.5-1.2 \mathrm{~cm}$. lata, supra nitidula subtus concoloria nitidula, apice breviter acuminata, basi acuta, nervis lateralibus numerosis tenuis prope ad marginem in nervo antemarginale coalitis. Racemi axillares solitarii $1-2 \mathrm{~cm}$. longi, rhachi glabra elongata, bracteae ovatae apice acutae circ. $1 \mathrm{~mm}$, longae; flores ô longe pedicellati, pedicelli circ. $3 \mathrm{~mm}$. longi, sepala 2 exteriora lanceolata apice acuminata, circ. $2.2 \mathrm{~mm}$. longa, $1 \mathrm{~mm}$. lata; 2 interiora ovata vel ovato-oblonga circ. $2.2 \mathrm{~mm}$. longa, $1.5 \mathrm{~mm}$. lata, ovarii rudimentum depresso-globosum integrum; stamina circ. $3.5 \mathrm{~mm}$. longa sepala superantia; flos o terminalis, sepala 2 exteriora ovata quam interiora paullo longiora, 4 interiora late ovata, acuta, circ. $2.2 \mathrm{~mm}$. longa, sparse ciliolata vel pubescentia. Fructus juniores ovoidei, glabri, $5-6 \mathrm{~mm}$. longi, styli prominentes, stigmata recurvata.

Philippine. Luzon: Guinatacutan, Tayabas, alt. 75-100 m., March 1911 (FoXWORTHY \& RAMOS; B. S. no. 13169; on rocks along the river; the flower white and greenish; type). 
Unfortunately I have not been able to see the type specimen of this species. However, so far as I can judge from the original description, this seems to bear a strong resemblance to $B$. Loheri MERR. from which it differs in the smaller lanceolate leaves with sharply acuminate apex.

3. Buxus Holttumiana Hatusima, sp. nov.

Buxus Rolfei VIDAL in Sched. in Herb. Bot. Gard Singapore.

Frutex glaber, ramuli hornotini tetragoni circ. $3 \mathrm{~mm}$. crassi, vetustiores obscure angulati albo-cinerascentes erosi longitudinaliter fissi, internodiis $3-4 \mathrm{~cm}$. longis. Folia coriacea petiolata, ovatooblonga rarius ovato-elliptica vel late lanceolata, $10-17 \mathrm{~cm}$. longa, 3-7.5 cm. lata, apice breviter acuminata basi cuneata vel breviter acuminata, margine deorsum haud revoluta, supra glabra nitida, subtus nitidula, nervis lateralibus $15-19$, utraque facie elevatis, distinctis, prope ad marginem in nervo antemarginale coalitis, costa media supra valde impressa subtus prominente elevata. Racemi

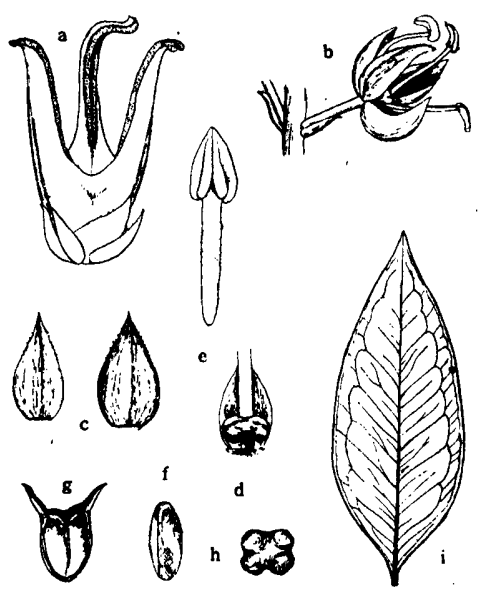

Fig. 1. Buxus Holltumiana Hatusima. $a$, female flower $\times 3,5$. b, part of inflorescence, bearing male flower $\times 4$. $c$, bracts of female flower, seen from without $\times 5$. $d$, part of male flower, showing rudimental ovary $\times 5$. e, stamen $\times 5$. $\mathrm{f}$, seed $\times 1$. g, valve of cap. sule $\times 1 / 2$. h, rudimental ovary, seen from above $\times 5$. $i$, leaf $\times 1 / 4$. axillares laxiflores in sicco nigrescentes, rhachi elongata glabra circ. $1 \mathrm{~cm}$. longa circ. $1 \mathrm{~mm}$. crassa, bractearum paria plerumque $4-5$, bracteae ovato-oblongae circ. 1.5 $\mathrm{mm}$. longae glabrae; flores $\hat{o}$ longe pedicellati (2-2.5 mm. longi), sepala ovata circ. $2.5 \mathrm{~mm}$. longa; stamina sepala superantia circ. $4 \mathrm{~mm}$. longa, antheris circ. $1.5 \mathrm{~mm}$. longis, ovarii rudimentum sepalis triplo breviore vix stipitatum circ. $1 \mathrm{~mm}$. diametro; flos o terminalis, sepala interiora ovata apice acuta circ. $2 \mathrm{~mm}$. longa, margine parce ciliolata, exteriora paullo angustiora anguste ovata circ. $2 \mathrm{~mm}$. longa, ovarium stylis complanatis apice leviter recurvatis vel erectis circ. duplo breviore, stigmata auguste oblanceolata fere usque ad basim stylum decurrentia. Capsula ob- 
ovoidea circ. $1.3 \mathrm{~cm}$. longa supra opaca, styli persistentes crassi apice haud recurvati circ. $6 \mathrm{~mm}$. longi; semina trigono-ellipsoidea circ. $8 \mathrm{~mm}$. longa lucida nigerrima. Fig. 1; Pl. I, Fig. 1.

Malay Peninsula: Perlis: Kaki Bukit, alt. 90 m., on limestone, April 11, 1938 (KIAT; S. F. no. 35239, in HS; shrub, fl. cream, fruit pink).

This is most closely related to Buxus Rolfeil VIDAL from the Philippines, but differs from it in the somewhat thicker shining leaves with fewer lateral nerves, in the longer not recurved styles with decurrent stigmas of the ovary, and in the different shape of the capsules with longer not recurved and much divaricated horns.

The species is named after Mr. R. E. HolTtuM, Director of the Botanic Gardens, Singapore, who kindly loaned me all specimens of Buxus preserved in the herbarium.

4. Buxus cochinchinensis PIERRE mss. ex GAGNEPAIN in Bull. Soc. bot. France, 481 (1921); in LecomTe, Fl. Gén. Indo-Chine 5: 663, fig. 77 (1927).

Frutex; ramulis angulatis, vetustioribus subteretibus longitudinaliter suberosis. Folia coriacea, lanceolata, apice acuminata vel mucronata, basi attenuata, 5-10 cm. longa, $1.5-3 \mathrm{~cm}$. lata, supra nitida, nervis lateralibus utrinsecus $10-13$, prope ad marginem in nervo antemarginale coalitis, basilaribus 3 , reticulis vix prominulis; petioli 2-4 mm. longi. Racemi terminales et axillares, bracteae acuminatae triangulares circ. $2 \mathrm{~mm}$. longae; flores $\hat{o}$ pedicellati, sepala 2 exteriora navicularia margine remote ciliata circ. $2.5 \mathrm{~mm}$. longa, 2 interiora late triangularia $2.5 \mathrm{~mm}$. longa, $2 \mathrm{~mm}$. lata, intus apicem versus sparse pilosula, stamina 4, sepala superatia, antheris introrsis oblongis apice obtusis circ. $1 \mathrm{~mm}$. longis, ovarii rudimentum depresso-tetragonum circ. $1.5 \mathrm{~m}$. latum; flos o terminalis, sepalà 7-8, triangularia acuta, ciliolata circ. $2 \mathrm{~mm}$. longa, ovarium ovoideum, styli 3 , valde recurvati, stigmata fere usque ad basim stylum decurrentia. Capsula ovoideo-globosa, 7-9 mm. longa, 8-9 $\mathrm{mm}$. lata, cornibus circ. $5 \mathrm{~mm}$. longis; semina nitida nigerrima circ. $6 \mathrm{~mm}$. longa.

Indo-China. Annam: Ba-rau, Prov. Phanrang; presqu'ile de Nui-han-heo, Prov. Nhatrang (PoILANE).-Cochin-China : montis Deonba ou Dinh? (PIERRE). 
I have not seen any specimen, but according to GAGNEPAin's description and figure in the literature cited above, this species seems to be closely related to Buxus Loheri MERR., from which it seems to differ in its more rounded capsules with longer horns.

5. Buxus Rolfei VIDAL, Rev. Pl. Vasc. Filip. 233 (1886).Ceron, Cat. Pl. Herb. Manila 147 (1892).-Merrill in Philip. Journ. Sci. 1: Bot. 84 (1906).-Halider f. in Meded. Rijks. Herb. 37 : 16 (1918).-MERRILL, Enum. Philip. Fl. Pl. 2: 465 (1923).

Frutex glaber, ramuli hornotini compresso-tetragoni circ. 1.5-2 $\mathrm{mm}$. diametro, vetustiores subteretes suberosi, internodiis $2-6 \mathrm{~cm}$. longis. Folia breviter petiolata, tenuiter coriacea, elliptica vel oblonga rarius ovato-lanceolata vel oblongo-lanceolata, $5-17 \mathrm{~cm}$. longa , (plerumque $6-16 \mathrm{~cm}$.) $1.5-8 \mathrm{~cm}$. lata (plerumque $2.5-4 \mathrm{~cm}$.), apice acuminata vel breviter acuminata, basi acuta, margine leviter deorsum recurvata, supra glabra nitida, subtus pallidiora glabra haud nitidula, nervis lateralibus numerosis utraque facie distinctis, prope ad marginem in nervo antemarginale coalitis, costa media supra impressa subtus valde elevata; petioli $2-7 \mathrm{~mm}$. longi (plerumque 2-3 $\mathrm{mm}$.), glabri. Racemi axillares laxiflores in sicco nigrescentes, rhachi glabra elongata 1-2 cm. longa (plerumque circ. $1 \mathrm{~cm}$.), circ. $1 \mathrm{~mm}$. crassa, bractearum paria 5 , bracteae ovatae acutae vel acuminatae $1.5-3 \mathrm{~mm}$. longae (plerumque 1.5-2 mm.) glabrae, margine angustissime scariosae ciliolatae; flores ô longe pedicellati $(2-5 \mathrm{~mm}$. longi), sepala ovato-

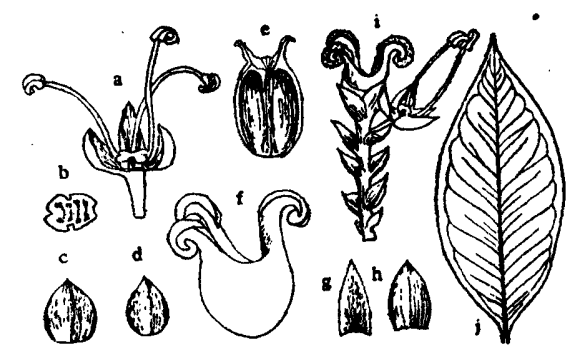

Fig. 2. Buxus Rolfei VIDAL (ELMER, no. 13239). a, male flower, sepal and stamen taken off $\times 3$. b, rudimental ovary, seen from above $\times 4$. c, $d$, outer and inner sepals of female flower $\times 4$. e, valve of capsule, seen from within $\times 1$. f, ovary $\times 3.5$. $\mathrm{g}, \mathrm{h}$, outer and inner sepals of male flower $\times 3$. i, inflorescence with male flower $\times 2$. $j$, leaf $\times 1 / 2$. 
elliptica, apice acuminata vel acuta, margine angustissime scariosa 2-3 mm. longa, stamina sepala superantia $3-5 \mathrm{~mm}$. longa, antheris circ. $1.5 \mathrm{~mm}$. longis, ovarii rudimentum sessile depresso-tetragonum sepalis triplo breviore; flos o terminalis, sepala interiora late ovata, apice acuta, margine angustissime scariosa et ciliolata, $1.8-3 \mathrm{~mm}$. longa, exteriora ovata, apice acuta, 2-3 $\mathrm{mm}$. longa, ovarium glabrum stylis complanatis apice spiro-recurvatis paullo breviore, stigmata lineari-obcordata usque ad basim stylum decurrentia. Capsula alutaceo-rugosa, opaca, $1-1.5 \mathrm{~cm}$. longa, styli persistentes crassi lanceolati circ. $2 \mathrm{~mm}$. longi; semina trigono-ellipsoidea circ. $7 \mathrm{~mm}$. longa nigerrima lucida. Fig. 2, Pl. II, Fig. 2.

Philippine. Luzon: Rizal: Mt. Susong-Dalaga, Aug. 1917 (M. RAmOS \& G. EDANO; B.S. no. 29423, in HA); without precise locality, Dec. 1912 (J. ReILLo; B.S. no. 19246, in HA); Bosobaso, Oct. 1904 (AHeR's collector; F.B. no. 1874, in HS). Tayabas: Mt. Dingalan, Aug.-Sept. 1916 (M. Ramos \& G. Eadano; S.B. no. 26625, in HA). Batan: Lamao River, Mt. Mariveles, May 1905 (BoRdEN; B.S. no. 3054 in HS). Palawan: Mt. Pulgar, Isl. Palawan, May 1911 (Elmer; S.B. no. 13239, in HA). Isl. Alabat, Sept.-Oct. 1926 (M. RAmos \& G. EdANo; B.S. no. 48352, in HA and HS: sub Buxus philippinensis ROLFE).

So far as I can know, Buxus philippinensis Rolfe was never published and appears merely in a manuscript.

6. Buxus Loheri Merrill in Philip. Journ. Sci. 9: Bot. 310 (1914); Enum. Philip. Fl. Pl. 2: 464 (1923).

Arbor glabra, ramuli fusco-rubescentes. Folia breviter petiolata, ovata-lanceolata .vel lanceolata, coriacea, $4.5-6 \mathrm{~cm}$. longa, $1.5-2 \mathrm{~cm}$. lata, apice acuminata, basi acuta, margine valde revoluta, supra nitidula subtus pallidiora plus minusve glaucescentia vel concoloria, nervis lateralibus utraque facie leviter prominentibus vel obsoletis, prope ad marginem in nervo antemarginale coalitis, costa media supra impressa subtus valde elevata; petioli circ. $2 \mathrm{~mm}$. longi glabri. Racemi axillares laxiflores solitarii glabri, rhachi glabra elongata circ. $7 \mathrm{~mm}$. longa, bractearum paria $4-5$, bracteae anguste ovatae, acutae, convexae glabrae, circ. $2 \mathrm{~mm}$. longae; flores $\hat{\delta}$ longe pedicellati (circ. $1.7 \mathrm{~mm}$. longi), sepala ovato-elliptica apice acuta circ. $2.5 \mathrm{~mm}$. longa, stamina sepala superantia circ. $3.5 \mathrm{~mm}$. longa, ovarii rudimentum depresso-tetragonum sepalis triplo bre- 


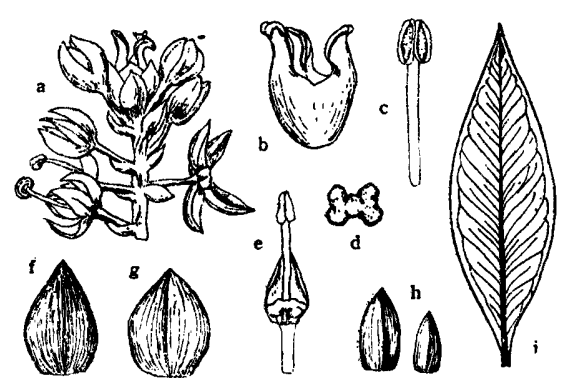

Fig. 3. Buxus Loheri MERr. (LOHER, no. 13496). a, inflorescence $\times 2 . \quad b$, ovary $\times 3.5 . \quad c$, stamen $\times 5$. d, rudimental ovary, seen from above $\times 4$. e, male flower, three sepals and stamens taken off $\times 3$. $f, g$, outer and inner sepals of female flower, seen from without $\times 3$. h, same of male flower, seen from without $\times 3$. i, leaf $\times 1 / 2$.

viore haud stipitatum; flos $\odot$ terminalis, sepala 2 exteriora ovata, acuta circ. $3 \mathrm{~mm}$. longa, 4 interiora quam exteriora paullo latiora, acuta circ. $3 \mathrm{~mm}$. longa; ovarium glabrum, stylis apice recurvatis paullo breviore, stigmata lineari-obcordata fere usque ad basim stylum decurrentia. Capsula ovoidea circ. $1 \mathrm{~cm}$. longa. Fig. 3 .

Philippine. Luzon: Rizal: Montalban, Feb. 1905 (LoHER, no. 6857; type, not seen); same province, Paningtingan, March 1915 (LOHER, no. 13496, in HA).

Of this species I have seen but a flowering specimen, LoHER, no. 13496, of which I have given a description above. This box is undoubtedly most closely related to Buxus Rolfei VIDAL, and I have been unable to discover any floral characters serving to differentiate these two species except that the former has somewhat smaller and thicker obscurely nerved leaves.

\section{Buxus sp.}

Buxus Rolfei sensu MerRill, Philip. Fl. Pl. 2: 465 (1923), pro parte, quoad spec. ex Mindanao.-non VIDAL.

Philippine. Mindanao: Surigao, June, 1119 (M. Ramos \& J. Pasgasio; B. S. no. 34726 , in $\mathrm{Ha}$ ).

This is the form referred by MerRILl (Enum. Philip. Fl. Pl. 2: $465)$ to Buxus Rolfei VIDAL, from which it differs in the much thicker coriaceous leaves with strongly revoluted margins, an acute apex, and scarcely elevated lateral nerves on both surfaces.

Unfortunately the material is very poor, so without having 
seen better materal with fully developed flowers, it is very difficult to decide whether this collection represents a new species or may refer to one of the species already known.

Sect. Eubuxus (Baillon) Hatusima, emend.

Subgen. Eubuxus Baillon, Monogr. Bux: (1859) t. 1.

Frutex vel arbor parva. Folia apice saepissime emarginata vel obtuse acuta rarius acuminata, pennivenia, costa media in sicco supra saepissime prominente elevata rarissime subplana vel leviter impressa, subtus elevata. Inflorescentiae racemosae vel spicatae, rhachi brevia vel elongata plus minusve pilosula rarius glabra; flores $\hat{o}$ sessiles vel pedicellati, ovarii rudimentum sepala fere aequans vel ea breviore; flos + terminalis, ovarium glabrum rarius dense pubescente, styli crassi vel complanati apice plus minusve recurvati, stigmata obcordata vel auguste obcordata stylis circ. duplo breviore vel fere usque ad basim stylum decurrentia. Capsula saepius ovoidea luciduscula rarius opaca. Species ad 23, Asiae et Europae incolae.

Though I. have studied almost all the species of Eubuxus, I find it impossible to divide this section into well characterized subsections or series. There are, however,groups of allied species which stand out more or less clearly, but I have not succeeded in characterizing these groups distinctly, becourse the characters are too variously distributed, and of many species important characters are still unknown owing to the want of sufficient material. To arrange, however, at least for the present paper, the Asiatic species in a way to indicate their affinities, I have distinguished certain groups without naming them.

1) Ovarii rudimentum floribus masculis vix dimidia sepala aequans.

2) Inflorescentiae racemosae laxiflores, rhachi plus minusve elongata.

3) Folia utrinque opaca glauca, costa media supra leviter impressa. ........................................... 1 Group.

3) Folia utrinque viridia rarius opaca, costa media supra valde elevata. ...................................... 2 Group.

2) Inflorescentiae spicatae vel racemosae dense glomeratae, rhachi brevissima.

3) Flores $\hat{o}$ longe pedicellati, ovarium stylis complantis apicem 
vérsus sensim angustatis multo breviore, stigmata anguste oblanceolata usque ad basim stylum decurrentia, ramuli subteretes.............................................. 3 Group.

3) Flores $\hat{o}$ sessiles, ovarium stylis crassis apicem versus vix angustatis paullo longiore, stigmata usque ad medium $2 / 3$ stylum decurrentia obcordata vel anguste obcordata.

4) Folia glauca papillosa, costa media supra subplana. ...... 4 Group.

4) Folia viridia epapillosa, costa media supra valde elevata. 5 Group.

1) Ovarii rudimentum floribus masculis sepala aequans vel fere aequans.

2) Inflorescentiae racemosae vel spicatae, rhachi plerumque plus minusve elongata rarius brevissima; folia oblanceolata vel spathulata rarius obovata saepissime supra medium latissima, costa media in sicco subtus acute elevata (excl. no. 21), nervis lateralibus subtus plus minusve distinctis (excl. no. 21).........................................6 Group.

2) Inflorescentiae spicatae dense glomeratae, rhachi brevissima; folia ovata vel ovato-lanceolata vel elliptica rarius oblanceolata vel obovata saepissime infra medium latissima, apice saepissime emarginata vel obtusa, costa media subtus obtuse elevata. 7 Group.

Conspectus specierum et varietarum sectionis Eubuxui.

1) Ovarii rudimentum in floribus masculis vix dimidia sepala aequans (in no. 15 et 19 no visi).

2) Ovarium stylis apicem versus sensim plus minusve angustatis distincte breviore, stigmata anguste obcordata.

3) Flores $\hat{o}$ distincte pedicellati.

4) Racemi laxiflores, rhachi plus minusve elongata; ramuli hornotini angulato-tetragoni subteretes.

5) Folia supra opaca, costa media in sicco supra leviter impressa, nervis lateralibus supra obsoletis; ramuli hornotini petiolique plus minusve farinoso-puberuli; styli plus minusve crassi apicem versus valde recurvati; pedicelli circ. $3 \mathrm{~mm}$. longi. ... 7, B. rupicola. 
5) Folia supra viridia nitidula, costa media in sicco supra valde elevata, nervis lateralibus supra distinctis; styli plus minusve complanati; pedicelli cir. $0.5-2.5 \mathrm{~mm}$. longi.

6) Ramuli petiolique glaberrimi.

7) Ramuli hornotini subteretes, folia oblongo-elliptica circ. $5-7 \mathrm{~cm}$. longa; stigmata usque ad medium stylum apice leviter recurvatum decurrentia, pedicelli circ. $1 \mathrm{~mm}$. longi.......... 7. B. megistophylla.

7) Ramuli hornotini angulato-tetragoni; folia lanceolata rarius elliptica circ. $7-11 \mathrm{~cm}$. Ionga; stigmata fere usque ad basim stylum apice valde recurvatum decurrentia; pedicelli circ. $2.5 \mathrm{~mm}$. longi. 9. B. hainanensis.

6) Ramuli petiolique plus minusve pubescentes.

7) Folia apice acutiuscula, nervis lateralibus in sicco utrinque plus minusve distinctis, costa media subtus abrupte valde elevata; capsula nitida ; pedicelli circ. $1 \mathrm{~mm}$. longi.

8) Folia oblanceolata vel spathulata vix ultra $2 \mathrm{~cm}$. longa, apice mucronata, utrinque distincte reticulata; stigmata satis brevia haud vel vix ad medium stylum tantum apice leviter recurvatum decurrentia....... 10. B. austro-yunnanensis.

8) Folia lanceolata vel elliptica plerumque ultra $4 \mathrm{~cm}$. longa; stigmata usque ad medium vel basim stylum apice valde recurvatum decurrentia.

9) Folia lanceolata, nervis lateralibus reticulisque distinctis; stigmata usque ad basim stylum apice valde recurvatum decurrentia ............ 11. B. Myrica.

9) Folia elliptica vel rhombeo-elliptica, nervis lateralibus reticulisque subtus obsoletis; styli apice leviter recurvati.

10) Folia ovata vel elliptica apice obtusiuscula $3-8 \mathrm{~cm}$. longa $1.5 \mathrm{~cm}$. lata; stigmata usque ad basim stylum decurrentia 
10) Folia rhombeo-elliptica apice obtuse acuminata $5-3 \mathrm{~cm}$. longa $1-3 \mathrm{~cm}$. lata; stigmata usque ad medium stylum decurrentia ........ 13. B. malayana.

7) Folia apice saepissime breviter emarginata rarius obtuse acuta, nervis lateralibus subtus obsoletis, costa media subtus sensim obtuse elevata; capsula opaca scabra.

8) Pedicelli circ. $0.7 \mathrm{~mm}$. longi; ovarii rudimentum circ. $0.6 \mathrm{~mm}$. longum; ovarium stylis paullo longiore. ....................... 14. B. liukiuensis.

8) Pedicelli circ. $1.7 \mathrm{~mm}$. longi; ovarii rudimentum circ. $1 \mathrm{~mm}$. longum; ovarium stylis circ. duplo breviore; folia paullo angustiora

B. liukiuensis var. longipedicellata.

4) Racemi dense glomerati, rhachi brevissima; ramuli hornotini subteretes glaberrimi; ovarium stylis complanatis apicem versus valde recurvatis gracilibus multo breviore; stigmata linearia usque ad basim stylum decurrentia; pedicelli circ. $2.5 \mathrm{~mm}$. longi; ovarii rudimentum apice vix dilatatum valde gracile; folia ovato-lanceolata vel oblongo-lanceolata $5-11 \mathrm{~cm}$. longa......... 16. B. Henryi.

3) Flores $\hat{\delta}$ sessiles: folia elliptica vel oblonga plerumque 4-5 $\mathrm{cm}$. longa utrinque opaca primo plus minusve pubescentia . mox glabrescentia.

4) Folia ramulique dense pubescentia...... 15. B. mollicula.

4) Folia ramulique glabrescentia.... B. mollicula var. glabra.

2) Ovarium stylis crassis apicem versus vix angustatis \pm longiore; inflorescentiae densissime glomeratae, rhachi brevissima.

3) Flores $\hat{o}$ sessiles.

4) Ramuli petiolique glaberrimi glauci ; folia anguste lanceolata vel anguste oblongo-lanceolata utrinque opaca glaucescentia subtus valde papillosa, costa media supra vix elevata, nervis lateralibus utrinque obsoletis; ovarii rudimentum apice haud dilatatum gracile; sepala bracteaeque flavescentia; capsula opaca vix nitidula.......... 17. B. papillosa. 
4) Ramuli petiolique plus minusve pubescentes; folia oblongo-lanceolata vel lanceolata vel ovato-lanceolata plerumque $4-5 \mathrm{~cm}$. longa supra viridia nitidula haud rugulosa subtus epapillosa, costa media supra valde elevata, nervis lateralibus supra distinctis; ovarii rudimentum crassum apice valde dilatatum.

5) Capsula glabra; folia oblongo-lanceolata vix ultra $1 \mathrm{~cm}$. lata, costa media subtus abrupte elevata.

18. B. Wallichiana.

5) Capsula dense adpresse pubescentia ; folia lanceolata vel ovato-lanceolata $1.5-2 \mathrm{~cm}$. lata. ...... 18. B. hebecarpa.

3) Flores 1 distincte breviter pedicellati; folia vix ultra 2 $\mathrm{cm}$. longa supra in sicco plus minusve rugulosa, nervis lateralibus supra obsoletis, costa media subtus leviter obtuse elevata; ramuli petiolique plus minusve pubescentes.

4) Folia supra glabra lucidiuscula viridia valde rugulosa, subtus flavo-viridescentia; ovarium glaberrimum.

5) Folia oblonga vel ovato-oblonga rarius elliptica vel ovata plerumque circ. $2 \mathrm{~cm}$. longa; caulis erectus vel ascendens. ..............................20. B. rugulosa.

5) Folia ovata vel ovato-elliptica plerumque vix ultra 1.5 $\mathrm{cm}$. longa; caulis prostratus vel ascendens.

6) Folia 1-1.3 cm. longa; ramuli. crassiores petiolique dense pubescentes; caulis ascendens vel erectus; capsula majora ut in typico. ...... var. intermedia.

6) Folia minora vix ultra $1 \mathrm{~cm}$. longa, ramuli graciles petiolique glabrescentes; caulis prostratus, ramis saepe radicantibus; capsula minora....... subsp. prostrata.

4) Folia anguste ovata vel oblongo-elliptica vel ovato-elliptica 1.5-2 cm. longa, supra opaca olivaceo-viridescentia sparsium pilosula mox glabra, subtus dense pilosula et plus minusve papillosa glaucescentia; fructus junioribus dense velutino-tomentellus. subsp. rupicola.

1) Ovarii rudimentum in floribus masculis sepala aequans vel fere aequans; ovarium stylis crassis apicem versus haud vel vix 
1

angustatis plerumque longiore; inflorescentiae dense glomeratae, rhachi brevissima (excl. no. 21).

2) Folia oblanceolata vel anguste spathulata rarius obovata vel anguste lanceolata saepissime supra medium latissima, apice plerumque breviter emarginata; ramuli hornotini petiolique plus minusive pubescentes; capsula minora plerumque vix ultra $4 \mathrm{~mm}$. lata; frutex humilis rarius mediocris.

3) Ovarii rudimentum sepalis paullo longiore vel aequans; ovarium glabrum.

4) Folia anguste obovata vix ultra $2 \mathrm{~cm}$. longa (plerumque $1.7 \mathrm{~cm}$.), costa media subtus vix elevata, nervis lateralibus utrinque obsoletis; flores $\hat{o}$ distincte pedicellati; ramuli petiolique pubescentes; racemi laxiflores, rhachi plus minusve elongata; frutex humilis.

5) Sepala bracteaeque dorso rubro-punctata; ovarium stylis crassis distincte longiore........... 21. B. ichangensis.

5) Sepala bracteaeque dorso vix rubro-punctata; ovarium stylo fere aequilongum; folia leviter longiora.

var. fukienensis.

4) Folia oblongo-oblanceolata vel spathulata vel anguste oblongo-oblanceolata rarius obovata circ. 2-4 cm. (plerumque $2.5-3 \mathrm{~cm}$.) longa, costa media subtus elevata supra in parte inferiore plerumque plus minusve puberula; ramuli petiolique plerumque plus minusve puberuli rarius glabrescentes; flores $\hat{\delta}$ breviter pedicellati vel sessiles; frutex mediocris.

22. B. Bodinieri.

3) Ovarii rudimentum circ. $2 / 3$ sepala aequans; frutex humilis.

4) Flores o distincte pedicellati; folia anguste oblanceolata vel oblanceolata circ. $1.7 \mathrm{~cm}$. longa, costa media subtus valde elevata, nervis lateralibus supra valde subtus leviter elevatis; ovarium glabrum. ... 23. B. Harlandii.

4) Flores of sessiles.

5) Fructus junioribus dense adpresse, tomentellus, folia anguste obovata vel spathulato-linearia rarius obovata circ. $0.5-2 \mathrm{~cm}$. (plerumque $1.5 \mathrm{~cm}$.) longa, costa media subtus leviter elevata, nervis lateralibus subtus plus minusve obsoletis. 24. B. cephalanthera. 
5) Ovarium glabrum; folia oblanceolata vel anguste obovata circ. 1-2 cm. longa, costa media subtus valde elevata, nervis lateralibus subtus distinctis.

25. B. stenophylla.

2) Folia ovata vel ovato-oblonga vel oblonga rarius lanceolata vel oblanceolata vel obovata plerumque infra medium latissima, costa media in sicco subtus leviter obtuse elevata, nervis lateralibus plerumque obsoletis; flores. $\hat{o}$ sessiles; fructus plerumque majoribus glaber; frutex altior.

3) Ramuli petiolique glabri.

4) Ramuli graciles dense ramosi; folia oblanceolata rarius obovata vel lanceolata, tenuiter coriacea.

26. B. microphylla.

4) Ramuli crassiores minus ramosi; folia coriacea vel crasse coriacea, ovata vel ovato-elliptica rarius obovata vel lanceolata. ....................................... var. japonica.

3) Ramuli petiolique plus minusve pubescentes.

4) Folia ovata vel ovato-oblonga vel elliptica rarius oblonga vel obovata apice plerumque emarginata vel obtusa; ovarii rudimentum sepala fere aequans, stamina sepalis circ. duplo longiora.

5) Folia ovata vel ovato-oblonga vel elliptica rarius oblonga vel obovata $1.3-5 \mathrm{~cm}$. longa, nervis lateralibus supra distinctis, margine leviter revoluta.

subsp. sinica.

5) Folia ovata vel ovato-oblonga vel elliptica vix ultra $2 \mathrm{~cm}$. longa, nervis lateralibus utrinque obsoletis, margine deorsum valde revoluta. ................. var. insularis.

4) Folia lanceolata vel ovato-lanceolata apice obtuse acuta 3-4 cm. longa, margine leviter revoluta; ovarii rudimentum circ. $2 / 3$ sepala aequans; stamina sepalis paullo longiora. var. aemulans.

\section{Group.}

Ramuli hornotini tetragoni farinoso-pubescentes; folia opaca costa media supra leviter impressa, nervis lateralibus utrinque ob- 
soletis; racemi laxiflores farinoso-pubescentes, rhachi elongata, flores of longe pedicellati, ovarii rudimentum sepalis multo breviore, sepala bracteaeque crassa, ovarium stylis subteretibus apice valde recurvatis paullo longiore, stigmata anguste obcordata fere usque ad basim stylum decurrentia; capsula ovoidea opaca. Species 1, Asiae tropicae incola.

The present group is most closely related to the group 4, but quite differs in the floral structure.

7. Buxus rupicola Ridley in Journ. As. Soc. S. Br. 59: 166 (1911); Fl. Malay Penins. 3: 182 (1924).

Frutex ramosus; ramuli hornotini obscure tetragoni dense breviter hirtelli circ. $1.5 \mathrm{~mm}$. crassi, vetustiores subteretes cinerascentes glabri longitudinaliter valde rugosi circ. $2 \mathrm{~mm}$. crassi, internodiis plerumque $1 \mathrm{~cm}$. longis. Folia opposita breviter petiolata, crasse coriacea, oblongo-lanceolata, oblonga vel ovato-oblonga, apice emarginata vel obtusa $3-6 \mathrm{~cm}$. longa $1.2-2.5 \mathrm{~cm}$. lata, basi cuneata, margine anguste revoluta, supra opaca haud nitidula glabra, subtus paullo pallidiora glabra, costa media supra leviter impressa basim

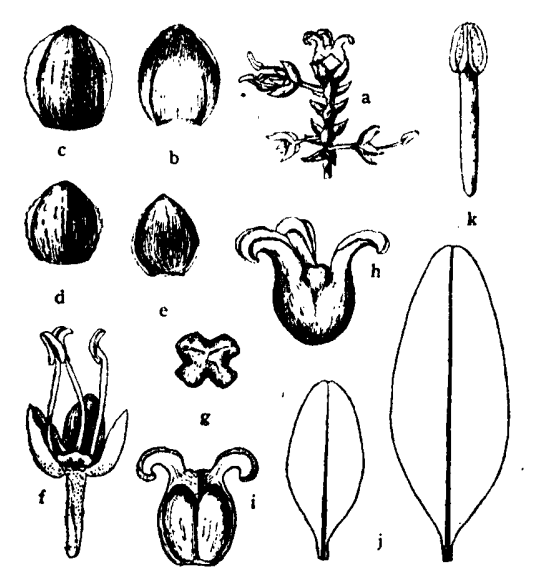

Fig. 4. Buxus rupicola Ridiey (Curtis no. 2662, type). $a$, inflorescence with three male flowers and female flower $\times 1.5$. b, c, ińner and outer sepals of male flower $\times 5$. d, e, same of female flower $\times 5$. f, male flower, sepal and stamen taken off $\times 4$. g, rudimental ovary, seen from above $\times 5$. h, ovary $\times 5$. i, valve of capsule $\times 2$. $\mathrm{j}$, leaves of different shapes $\times 1 / 2$. $\mathrm{k}$, stamen $\times 5$. 
versus sparse hirtella, subtus obtuse elevata glabra, nervis lateralibus utraque facie obsoletis ; petiolo circ. $2 \mathrm{~mm}$. longo hirtello. Racemi axillares laxiflores, rhachi elongata dense hirtella 6-8 $\mathrm{mm}$. longa circ. $1 \mathrm{~mm}$. crassa, bractearum paria $4-5$, bracteae ovatae acutae dorso densiuscule hirtellae circ. $1.5 \mathrm{~mm}$. longae; flores $\hat{o}$ pedicellati, pedicellis circ. $2-2.5 \mathrm{~mm}$. longis sparse hirtellis, sepala late ovalia circ. $2 \mathrm{~mm}$. longa margine anguste scariosa dorso basim versus sparse hirtella intus apicem versus sparse hirtella, stamina sepala superantia circ. $3.5 \mathrm{~mm}$. longa, antheris circ. $1 \mathrm{~mm}$. longis, ovarii rudimentum sepalis multo breviore haud stipitatum; flos $q$ terminalis, sepala late ovata circ. $1.5 \mathrm{~mm}$. longa apice obtuse. acuta, margine anguste scariosa, intus apicem versus. dorso basin versus sparse hirtella vel fere glabra, ovarium stylis subteretibus apice valde recurvatis paullo longiore (ovarium circ. $1.5 \mathrm{~mm}$. longum, styli circ. $1 \mathrm{~mm}$. longi), stigmata fere usque ad basim stylum decurrentia. Capsula ovoidea circ. $7 \mathrm{~mm}$. longa opaca haud nitida, styli persistentes crassi valde recurvati circ. $1 \mathrm{~mm}$. longi, semina ignota. Fig. 4, Fig. XI, Fig. 2.

Malay Peninsula. Kedah: Lankawi, near Dayong Bunting, on bare rocks by the sea, Sept. 1890 (CurTIS, no. 2662, type, in HS).

This interesting species somewhat resembles $B$. mollicula var. glabra HANDEL-MAZZETTI from western China and $B$. papillosa SCHNEIDER from the Himalayas in its dull upper surface of the obscurely nerved leaves, but quite differs in its floral structure and is much more similar to those of species belonging to Sect. Eugeniobuxus.

\section{Group.}

Ramuli tetragoni plerumque plus minusve puberuli rarius glabri; folia apice plerumque acutiuscula vel mucronulata rarius breviter emarginata supra nitida, costa media utrinque valde elevata, nervis lateralibus supra saepe distinctis; racemi plus minusve laxiflores, rhachi elongata puberula, flores $s$ pedicellati, ovarii rudimentum sepalis multo breviore; ovarium stylis complantis apicem versus sensim angustatis et recurvatis breviore glabrum, stigmata anguste obcordata vel lineari-obcordata plerumque usque ad medium vel basim decurrentia. Species 8, Chinae occidentali-australis, peninsulae Malayae, Cochinchinae et Archipelagi Liukiuani incolae. 
8. Buxus megistophylla LÉveILLÉ, Fl.' Kouy-Tchéou 160 (1914); Cat. Ill. Pl. Seu-Tchouen, pl. 26 (1918).-GAGNEPAIN in LECOMTE, Fl. Gén. Indo-Chine 5: 661 (1927).-ReHDER in Journ. Arn. Arb. 14: 236 (1933); 18 : 215 (1937).

Frutex glaber circ. $60 \mathrm{~cm}$. altus, ramuli compresse subteretes obscure angulati circ. $2 \mathrm{~mm}$. diametro, glabri, internodiis circ. 2 $\mathrm{cm}$. longis. Folia glabra subcoriacea oblonga vel ovato-oblonga apice obtuse acuta basi acuta 4-8 cm. longa (plerumque 5-7 $\mathrm{cm}$.), supra nitidula glabra subtus pallidiora, costa media supra leviter subtus manifeste elevata, nervis lateralibus reticulisque supra haud subtus vix conspicus; petiolo crasso $2-3 \mathrm{~mm}$. longo intus puberulo vel fere glabro. Racemi axillares laxiflores circ. $1 \mathrm{~cm}$. longi, rhachi pilosula vel fere glabra elongata 5-7 mm. longa; bractearum paria 6-7, bracteae ovatae apice acutae dorso basim versus pilosulae rubescentes margine tantum anguste scariosae; flores $\hat{o}$ paria 5 , breviter pedicellati, pedicellis circ. $0.8 \mathrm{~mm}$. longis, sepala exteriora rotundata dorso glabra 2-2.5 mm. lata, interiora quam exteriora paullo minora circ. $2 \mathrm{~mm}$. longa dorso glabra, stamina sepalis longiora circ. $6 \mathrm{~mm}$. longa, antheris circ. $1.5 \mathrm{~mm}$. longis; ovarii rudimentum sepalis circ.

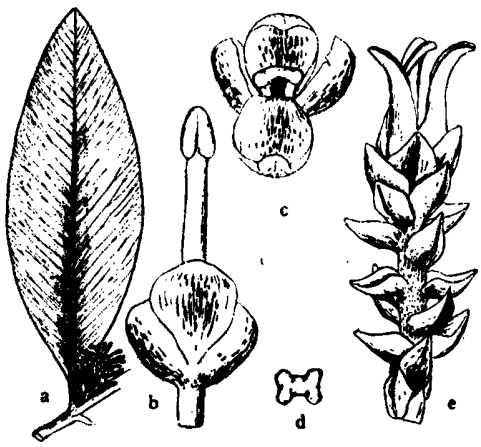

Fig. 5. Buxus megistophylla LÉvL. (BoDrNIER, no. 2607, type). a, branchlet with inflorescence $\times 1 / 2$. b, male flower with stamen $\times 5$. c, same, showing rudimental ovary $\times 5$. d, rudimental ovary, seen from above $\times 5$. e, inflorescence, male flowers taken off $\times 4$. duplo breviore circ. $1 \mathrm{~mm}$. longum; flos $\&$ terminalis, sepala ovato-elliptica circ. $3 \mathrm{~mm}$. longa dorso glabra; ovarium stylis apice leviter recurvatis paullo breviore (ovarium $2 \mathrm{~mm}$. longum, styli $2.5 \mathrm{~mm}$. longi), stigmata usque ad medium stylum decurrentia. Capsula ignota. Fig. 5.

China. Kweichou: environs de Hoang-ka-chou, rocailles de la cascade, March 15, 1899 (J. Sequin in herb. BodinIER, no. 2607; arbuste de $0.6 \mathrm{~m}$., fleurs blanches; syntype; merotype in HA); Kiao-men, prés Lo-fou, Dec. 1910 (J. EsquiRol, no. 2560, syntype; photo. in HA). 
In its floral structure this is most closely related to $B$. mollicula SM., but differs from it in the staminate flowers with distinct pedicels, in the glabrous almost rounded branchlets with much larger glabrous leaves; in its large glabrous leaves and almost rounded branchlets, 'this resembles also $B$. Henryi MAYR which has quite different flowers.

9. Buxus hainanensis MerriLl in Lingnan Sci. Journ. 14: 25, fig. 8 (1935).

Frutex glaber, rami teretes vel obscure angulati, ramuli compressi vel sulcati circ. $1 \mathrm{~mm}$. diametro, glaberrimi, internodiis 2-6 cm. longis. Folia subcoriacea vel chartacea, oblongo-elliptica vel oblongo-lanceolata, utrinque angustata, apice obtuso-acuta, basi acuta, utrinque nitidula, stricte penninervia, $7-11 \mathrm{~cm}$. longa, $2-3.5 \mathrm{~cm}$. lata, brevissime petiolata (circ. $1 \mathrm{~mm}$.), hargine paullo revoluta, costa media utrinque valde elevata, nervis lateralibus utrinsecus 15-18, gracilibus, distinctis, furcatis vel simplicibus, reticulis obliquis.

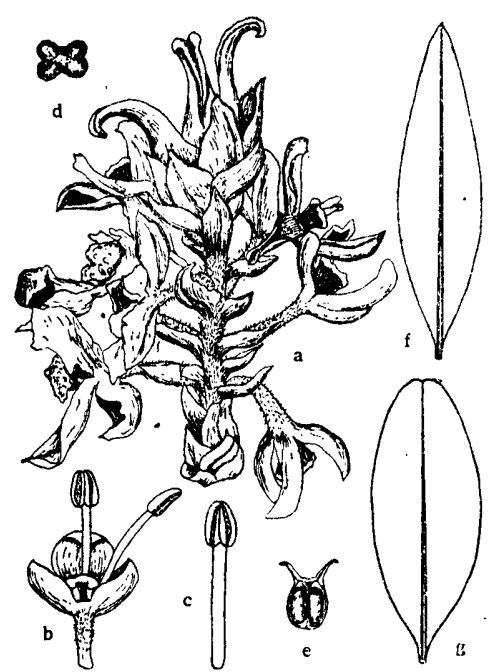

Fig. 6. Buxus hainanensis MERR. a, inflorescence $\times 4$. b, male flower, sepal and two stamens taken off $\times 3$. c, stamen $\times 5$. d, rudimental ovary, seen from above $\times 3.5$. e. valve of capsule, seen from within $\times 1 / 2$. $f, g$, leaves of different shapes $\times 1 / 2$.
Racemi axillares 7-12 $\mathrm{mm}$. longi laxiflores, rhachi dense pilosa elongata circ. 5-12 $\mathrm{mm}$. (plerumque circ. $8 \mathrm{~mm}$.) longa, bractearum paria $6-8$, bracteae, acutae, $2 \mathrm{~mm}$. longae dorso pilosulae margine ciliolatae; flores $\hat{o}$ saepissime paria 6 , longe pedicellati, pedicellis $2-2.5$ $\mathrm{mm}$. longis dense pilosis, sepala interiora ovata circ. $2 \mathrm{~mm}$. longa dorso glabra, exteriora quam interiora paullo angustiora glabra circ. $1.5 \mathrm{~mm}$. longa, stamina sepala superantia circ. $10 \mathrm{~mm}$. longa, ovarii rudimentum vix stipitatum sepalis 2-3 plo breviore $1.5 \mathrm{~mm}$. longum, $1 \mathrm{~mm}$. latum; flos of terminalis, sepala ovato-elliptica apice obtusoacuta dorso sparse pilosula, margine ciliata, ovarium stylis complanatis apice valde recurvatis circ. duplo breviore (ovarium $1.5 \mathrm{~mm}$. longum, 
styli $3 \mathrm{~mm}$. longi), stigmata fere usque ad basim stylum decurrentia. Capsula ovoidea usque $1 \mathrm{~cm}$. longa, stylis persistentibus crassis, lanceolatis, rigidis circ. $5 \mathrm{~mm}$. longis, curvatis. Fig. 6 .

China. Isl. Hainan: Sha-po-shan and vicinity, Taam-chau District, Aug. 1927 (Tsang, WaI-TAK, nos. 561, 596, in HA); Yaichow, alt. $450 \mathrm{~m}$., March-July, 1933 (F. C. How, no. 70527; shrub, ht. 60 cm., in HA); same District, woodside or along the stream, JulyOct., 1933 (H. Y. LIANG,. no. 62029; shrub, ht. $1 \mathrm{~m}$. or more in HA); Tung-koo-shan and vicinity near Shan-hoi village, Wen-ch'ang District, dry, gentle slope, clay, rocky, scattered shrub, rare, Aug. 425, 1932 (FunG, no. 20373, in HA); without precise locality, July 26, 1933 (C. WANG, no. 33344, in HA; small shrub, at margine of streams; fourth Hainan Expedition of Sun Yatsen University, July 1933-July 1934); Chun-kon, alt. 260 m.; June-July 1935 (L. LINSLET Gressitt, no. 1035 ; shrub $2 \mathrm{~m}$. ht., in HA); Hung-mo-shan and vicinity, Lai (Loi) area Ravines, Aug. 13, 1929 (Tsang \& Fung, no. 699 ; shrub $2 \mathrm{~m}$. ht., in HA).

Kwangtung: Lantau island, in a big ravine, Dec. 25, 1927 (TSANG, Herb. Lingn., no. 16582 ; vernacula name "Kit-ts'ing-shue," in $\mathrm{HA}$ ).

In its floral structure $B$. hainanensis MerR. resembles $B$. Myrica LÉVL.. and $B$. malayana RIDLEY, but differs from the first in the glabrous habit, in the longer pedicels of the male flowers, and in the much thicker and larger leaves with scarcely reticulated veins beneath; from the second in the glabrous habit and longer pedicels of the male flowers.

TSANG specimen from Lantau island, Kwangtung is an interesting Box which differs from the Hainan specimens in the somewhat thicker leaves with longer petioles and a narrower base. Unfortunately, however, the flowers are too young to decide whether this specimen represents a distinct species or merely a form of $B$. hainanensis MERR.

10. Buxus austro-yunnanensis Hutusima, sp. nov.

Frutex humilis prostratus. Ramuli tetragoni angulati puberuli circ. $1 \mathrm{~mm}$. diametro. Folia subcoriacea anguste obovata vel oblanceolata, apice mucronulata vel emarginata, basi sensim angustata, margine leviter anguste revoluta, $2-3.5 \mathrm{~cm}$. longa (plerumque 2-3 $\mathrm{cm}$.), 4-8 $\mathrm{mm}$. lata, costa media utrinque valde elevata supra in 
parte inferiore plerumque sparse puberula vel fere glabra, nervis lateralibus utrinque manifeste elevatis et distinctis. Racemi axillares laxiflores, rhachi valde elongata circ. $1 \mathrm{~cm}$. longa, dense pilosula; bractearum paria 5-6, bracteae acutae, chartaceae, margine auguste scariosae dorso dense pilosulae $1.5-2 \mathrm{~mm}$. longae; flores $\hat{o}$

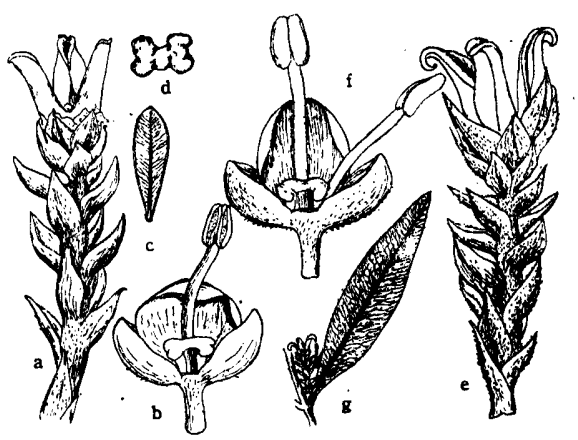

Fig. 7. Buxus austro-yunnanensis Hatusima, a-d, (Rock, no. 2528), Buxus Myrica LÉvl., e-g, (CAVAl ERIE, no. 3198). a, inflorescence, after male flowers fallen $\times 4$. b, male flower, inner sepal and three stamens taken off $\times 5$, c, leaf, seen from beneath $\times 1 / 2$. $d$, rudimental ovary, seen from above $\times 4.5$. e, inflorescence, male flowers taken off $\times 4$. $\mathrm{f}$, male flower, inner sepal and two stamens taken off $\times 5$. g, branchlet with inflorescence bearing male and female flower $\times 1 / 2$.

breviter pedicellati (circ. $1 \mathrm{~mm}$.), sepala late ovata glabra circ. 3 $\mathrm{mm}$. longa, stamina sepala superantia, ovarii rudimentum sepalis 2-3 plo breviore circ. $1.5 \mathrm{~mm}$. longum, apice valde dilatatum; flos o terminalis, sepala ovata circ. $3 \mathrm{~mm}$. longa, $2.5 \mathrm{~mm}$. lata, glabra, ovarium stylis complanatis apicem versus semsim angustatis leviter recurvatis $2-3$ plo breviore, stigmata anguste oblanceolata stylis circ. 3-4 plo breviora. Capsula ignota. Fig. 7 a-d, P1. II, Fig. 1.

China. Southern Yunnan: near Keng Hung, Feb. 22, 1922 (Rock, no. 2528; small prostrate bush, river-bed of the Meh-Kong among rocks, submerged for several months, in HA).

This is most closely related to B. Myrica LÉvL., from which it differs in the smaller and thicker subcoriaceous leaves with mucronulate or obtuse apex, and in the scarcely reflected styles with very short and narrower stigmas. 
11. Buxus Myrica LÉveillé in Fedde, Repert. Sp. Novi. 11: 549 (1913); Fl. Kouy-Tchéou 160 (1914).-GAGNEPAIN in LECOMTE, Fl. Gén. Indo-Chine, 5: 662 (1927).-REHDER in Journ. Arn. Arb. 14: 236 (1933); 18: 215 (1937).

Frutex ramulis tetragonis breviter pilosis, gracilibus: Folia brevissime petiolata, petiolis pilosulis, oblongo-lanceolata vel anguste lanceolata, $2-5 \mathrm{~cm}$. longa et $0.5-4 \mathrm{~cm}$. lata, acuta, mucronulata, basi, cuneata, glabra, tenuiter coriacea et in sicco utrinque distincte reticulata, costa media utrinque elevata. Racemi axillares laxiflores numerosi, rhachi dense pilosula elongata $6-7 \mathrm{~mm}$. longa ; bractearum paria $6-8$, bracteae ovatae, acutae, $2 \mathrm{~mm}$. longae, dorso dense pilosulae et intus ad marginem villosulae; flores $\hat{o}$ breviter pedicellati, pedicelli $1-1.5 \mathrm{~mm}$. longi dense pilosuli, sepala ovata glabra circ. $3 \mathrm{~mm}$. longa, stamina sepala superantia $4 \mathrm{~mm}$. longa, ovarii rudimentum sepalis triplo breviore; flos of terminalis, sepala oblongo-ovata 3-4 $\mathrm{mm}$. longa, 3 exteriora dorso dense 3 interiora sparse pilosula, ovarium stylis complanatis apice recurvis multo breviore (ovarium $1.5 \mathrm{~mm}$. longum, styli $3.5 \mathrm{~mm}$. logi), stigmata anguste oblanceolata ad medium vel $2 / 3$ stylum decurrentia. Capsula non visa. Fig. 7 e-g.

China. Kweichow: Pin-fa, April 8, 1907. (J. CAvalerie, no. 3198, syntype; merotype in HA); Lo-hou, Dec. 1910 (J. EsQUIROL, no. 2566, ex LÉveILlé syntype). Indo-China: Tonkin (fide GaGNePAIN).

var. angustifolia GaGNePAin in Lecomte, Fl. Gén. Indo-Chine 5: 662 (1927).

A typo recedit ramulis internodiis abbreviatis, foliis angustioribus $2.5-5 \mathrm{~cm}$. longis, $0.5-1 \mathrm{~cm}$. latis, bracteae ovatae acuminatae circ. $3 \mathrm{~mm}$. longae, ovarium stylis apice acutis circ. $2.5 \mathrm{~mm}$. longis breviore, stigmatibus linearibus ad medium stylum decurrentibus.

China. Kweichow: Tchen-lin, grande cascade de Hoang-kochou, dans les rochers, April 1898 (J. SEQUIN, in herb. BodINIER, no. 2266, syntype; photo. in HA). Indo-China: Tonkin (fide GAGNEPAIN).

12. Buxus latistyla Gagnepain in Buil. Soc. bot. France, 482 (1921); in LecomTe, Fl. Gén. Indo-Chine, 5: 661, fig. 77, 78 (1927).

Frutex ramis teretibus striatis, ramuli tetragoni longitudinaliter 
sulcati graciles. Folia ovata apice acuminata vel acuta, basi rotundata, coriacea, $3-8 \mathrm{~cm}$. longa, $1.5-3 \mathrm{~cm}$. lata, nervis lateralibus parallelis utrinsecus $15-18$, a se $2.4 \mathrm{~mm}$. remotis apice bifurcatis vel simplicibus; petioli circ. $8 \mathrm{~mm}$. longi. Racemi axillares vel : subterminales saepissime oppositi circ. $8 \mathrm{~mm}$. longi ambitu ovoidei 5-6 mm. lati; bracteae ovatae dorso pilosulae circ. $4 \mathrm{~mm}$. longae; flores $\hat{s}$ breviter pedicellati (circ. $1 \mathrm{~mm}$.), sepala 2 exteriora ovata acuta valde convexa doso puberula circ. $3 \mathrm{~mm}$. longa, 2 interiora ovato-elliptica glabra, stamina 4 , filamentis $5 \mathrm{~mm}$. longis, antheris ellipticis apice apiculatis circ. $1 \mathrm{~mm}$. longis, ovarii rudimentum depresso-tetragonum circ. $1 \mathrm{~mm}$. latum; flos ㅇ terminalis, ovarium circ. $5 \mathrm{~mm}$. longum, styli divaricates compressi, stigmata oblonga canaliculata usque ad basim stylum decurrentia circ. 1.2 $\mathrm{mm}$. longa. Capsula globosa circ. $8.5 \mathrm{~mm}$. diametro, cornibus 3.5 $\mathrm{mm}$. longis vix compressis; semina trigono-ellipsoidea nigerrima circ. $6.5 \mathrm{~mm}$. longa.

Indo-China. Tonkin: Prov. de Nam-dinh, entre Day-dong et Da-han (Chevalier). Laos: (Massie). Annam: Prov. de Than-hoa, à Diên-ho (BoN); Mai-lanh, Prov. Quang-tri (PoIlanE).

Of this species I have not seen any specimen, but according to GANGNEPAIN's description and figures, this must be most closely related to $B$. malayana RIDLEY.

13. Buxus malayana RIDLEY in Kew Bull. Misc. Inform. 475 (1926).

Frutex ramosus, ramuli hornotini tetragoni dense pilosuli circ. $1.2 \mathrm{~mm}$. crassi, angulis demum suberosis, internodiis plerumque circ. $2 \mathrm{~cm}$. longis. Folia breviter petiolata, tenuiter coriacea, ovatolanceolata rarius lanceolata $5-8 \mathrm{~cm}$. longa $1-3 \mathrm{~cm}$. lata, apice acuminata sed ad summum mucronata, rarius obtuse acuminata vel acuta, basi cuneata, margine leviter anguste recurvata, utraque facie glabra, supra viridia nitidula subtus opaca pallidiora, costa, media utraque facie valde acuto-elevata, supra basim versus pilosula subtus glabra, nervis lateralibus supra distinctis subtus haud distinctis; petiolo circ. $2 \mathrm{~mm}$. longo dense breviter pilosulo. - Racemi terminales et axillares glomerati ovoidei circ. $1 \mathrm{~cm}$. lati, rhachi dense pilosula primo circ. $3-4 \mathrm{~mm}$. longa sed post anthesin plus minusve elongata, bractearum paria circ. 4 , bracteae ovatae naviculares apice acutae tenuiter coriaceae circ. 2-3 mm. longae dorso sparse pilosulae, 
margine ciliolatae; flores $\hat{o}$ pedicellati, pedicelli circ. $1 \mathrm{~mm}$. longi sparse piosuli, sepala interiora fere orbicularia convexa circ. $2 \mathrm{~mm}$. longa, margine anguste scariosa dorso basim versus sparse pilosula, exteriora navicularia dorso ad costam sparse pilosula circ. $2 \mathrm{~mm}$. longa, stamina sepala superantia circ. $3 \mathrm{~mm}$. longa, antheris circ. $1 \mathrm{~mm}$.

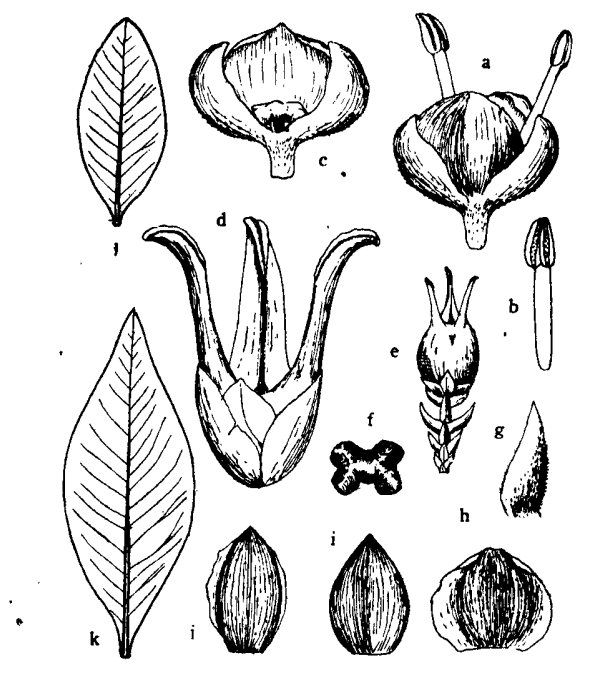

Fig. 8. Buxus malayana RidLey (type). a, male flower, two stamens takenoff $\times 5$. b, stamens $\times 5$. c, male fiower, showing rudimental ovary $\times 5$. d, female flower $\times 5$. e, inflorescence bearing capsule $\times 5$. f, rudimental ovary, seen from above $\times 5$. g, bract, seen from side $\times 5$. h, inner sepal, seen from without $\times 5$. $i, j$, outer and inner sepals of female flower, seen from outside $\times 5 . \mathrm{k}, \mathrm{l}$, leaves of different shapes $1 / 2$.

longis, ovarii rudimentum sepalis triplo breviore; flos $q$ terminalis, sepala interiora ovalia convexa circ. $2.5 \mathrm{~mm}$. longa dorso basim versus sparse pilosula, interiora ovato-oblonga convexa margine ciliata anguste scariosa dorso glabra circ. $2.5 \mathrm{~mm}$. longa, ovarium stylis complanatis apice recurvatis multo breviore, stigmata anguste oblanceolata usque ad medium stylum decurrentia. Capsula ovoidea laevia circ. $8 \mathrm{~mm}$. lata, styli persistentes rigidi circ. $5 \mathrm{~mm}$. longi, semina trigono-elliptica circ. $5 \mathrm{~mm}$. longa nigerrima nitida. Fig. 8 , Pl. X, Fig. 1. 
Malay Peninsula. Perak: Gunong lanok, near Batu Gajah, alt. 250 m., on limestone, April 16, 1925 (G. R. Mills \& M. R. HendersoN, no. 15078, type in HS). Selangor: Batu Takun, near Kanching, on limestone, Nov. 3, 1937 (NuR, S.F., no. 34382, in HS).

RIDLEY compared this Box with B. mollicula SM. from western China with which it resembles in no respect. This seems to be most closely related to $B$. latistyla GAGNEPAIN from Cochin-China, of which I have seen no specimens, but according to GAGNEPAIN's description and figures, the present species seems to differ from it in the somewhat narrower and larger leaves, and in the longer styles with much shorter stigmas. Both species need further investigation. This is certainly also closely related to B. Myrica LÉvEILLÉ from southern China, but differs from it in the much longer and thicker leaves with less reticulated nerves on the under surface.

14. Buxus liukiuenis MakiNo in Bot. Mag. Tokyo 16: 179 (1902).-SCHNEIDER, Illus. Handb. Laubholzk. 2: 140 (1907)-REHDER et Wilson in SargenT, Pl. Wils. 2: 168 (1914).-SaKaguti, Gen. Ind. Fl. Okinawa, 45 (1924).-Makino et Nemoto, Fl. Jap. 630 (1925) ; ed. 2, 663 (1930).-SASAKI in Trans. Nat. Hist. Soc. Formos. 18: 179 (1928); List Pl. Formos. 267 (1928).-S. Suzuki in MaSAMUNE, Short Fl. Formos. 126 (1936).

Buxus sempervirens sensu HeMsLEY in Journ. Linn. Soc. 26: 418 (1894), pro parte.-KaWAKAMI et SASAKI in Trans. Nat, Hist. Soc. Fomos. 22: 15 (1915)-non LinNaEUs.

Buxus sempervirens var. liukiuensis Makino in Bot. Mag. Tokyo, 9: 279 (1895); 15: 169 (1910).

Buxus Wallichiana sensu Hayata, Rev. Euphorb. et Buxac. in Journ. Coll. Sci. Imp. Univ. Tokyo, 20, art. 2: 84, t. E (1904).Matsumura, Ind. Pl. Jap. 2 (2): 311 (1912)-non BaIlLon.

Buxus microphylla var. sinica sensu HaYATA, Gen. Ind. Fl. Formos. 65 (1916) pro parte.-non REHDER et WiLson.

Buxus intermedia KaneHIRA, Formos. Trees rev. ed. 359, fig. 315, B. et C (1936) pro parte.

Frutex vel arbor parva. Ramis obscure angulatis albo-cinerascentibus, ramuli hornotini compresse tetragoni plus minusve pubescentes circ. 1-2 mm. diametro. Folia coriacea vel tenuiter coriacea, ovato-elliptica vel ovato-oblonga vel ovato-lanceolata rarius oblonga, 
apice saepissime emarginata rarius mucronata vel obtuso-acuta, basi acuta vel attenuata, margine leviter recurvata $3-6 \mathrm{~cm}$. longa, 1-3 cm. lata, supra nitidula glabra, subtus opaca, costa media utrinque manifeste elevata supra in parte inferiore sparse pilosa vel fere glabra, nervis lateralibus supra distictis subtus vix conspicuis; petiolo supra plano circ. $2 \mathrm{~mm}$. longo, margine plus minusve piloso. Racemi axillares laxiflores ambitu ovoidei, rhachi plus minusve elongata circ. $5 \mathrm{~mm}$. longa dense pilosa; bractearum paria 5-6, bracteae ovato-oblongae convexae circ. $2 \mathrm{~mm}$. longae dorso plus minusve pilosulae intus apicem versus pilosulae; flores $\hat{o}$ breviter pedicellati $(0.5-1 \mathrm{~mm}$.), sepala interiora orbicularia vel ovato-elliptica circ. $2 \mathrm{~mm}$. longa, interiora quam exteriora paullo angustiora dorso pilosula, stamina sepala superantia circ. $5 \mathrm{~mm}$. longa, ovarii rudimentum subsessile sepalis 2-3 plo breviore circ. $0.6 \mathrm{~mm}$. longum, apice dilatato-tetragonum 4-sulcatum; flos 9 terminalis, sepala orbicularis $3-3.5 \mathrm{~mm}$. longa convexa membranacea, ovarium stylis

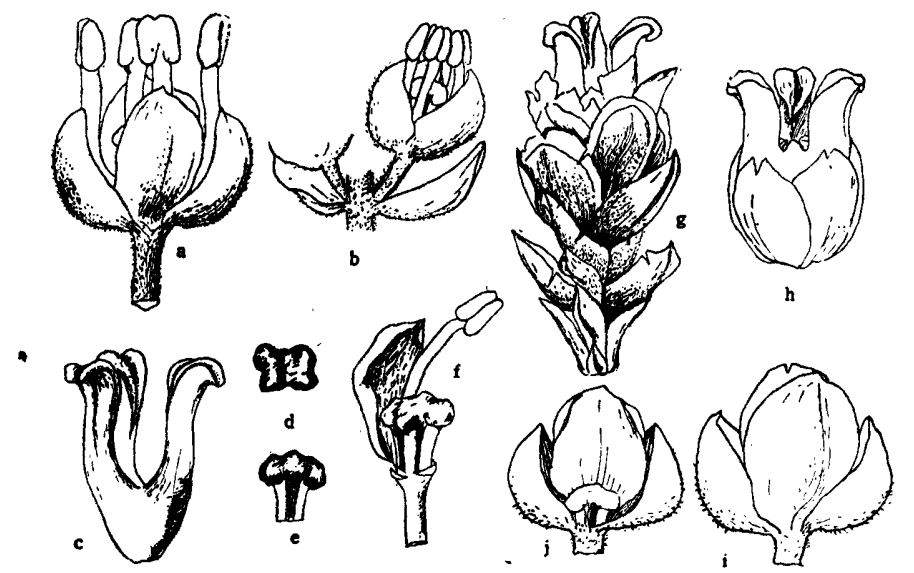

Fig. 9. Buxus liukiuensis Makino (g-j), var. longipedicellata Hatusima (a-f). a, male flower $\times 4$. b, portion of inflorescence $\times 2.5$. c, ovary $\times 5$. $d$, e, rudimental ovary, seen from above and side $\times 4$. f, male flower, three stamens and sepals taken off $\times 4 . \mathrm{g}$, inflorescence, male flowers taken off $\times 3.5$. h, female flower $\times 4$, i, male flower, stamens taken off $\times 4, j$, same, showing rudimental ovary $\times 4$,

complanatis apice recurvatis paullo longiore, stigmata fere usque ad basim stylum decurrentia. Capsula subglobosa in sicco opaca cum stylis persistentibus crassis $2-3 \mathrm{~mm}$. longis circ. $6 \mathrm{~mm}$. longa. Fig. $9 \mathrm{~g}-\mathrm{j}$. 
Japan. Liukiu: Isl. Amami-ohsima, 1887 (WARBURG, in HA; cultivated?). Isl. Okinawa, Kunigamigun, Jan. 30, 1924 (Z. TASIRO), same district, Jan. 3, 1934 (KANEHIRA, no. 3215); Nawa, 1907 (T. KAWAKAMi et G. NAKaHARA, in HA); Isl. Aka, Harama group, March 2, 1917 (WILSON, no. 8019; shrub 6-10 ft. $\times 1 \mathrm{ft}$., wild, in HA). Isl. Yonakuni, Oct. 1917 (Y. SimadA, Herb. Taihoku, nos. 19109, 15108, in HT).

Formosa: Hokuto, Toroku, Prov. Taihoku, March 17, 1909 (Y. SimadA, Herb. Taihoku, no. 15114; HT et HA); Apes Hill, Takao (A. HeNRY, no. 1177; shrub 1-2 ft., in HA).

This Box is fairly common in Liukiu Archipelago and Formosa, and often planted in Liukiu as hedges. This seems to be most closely related to $B$. hainanensis MERR., from which it differs in the pubescent branchlets, in the shorter pedicels of the male flowers with somewhat longer rudimental ovaries, in the less recurved styles, and in the not shining scabrous capsules.

var. longipedicellata HATUSIMA, var. nov.

A typo recedit foliis paullo minoribus angustioribus, nervis lateralibus densioribus utrinque distinctis, pedicellis longioribus circ. $1.7 \mathrm{~mm}$. longis, ovarii rudimentum paullo longiore circ. $1 \mathrm{~mm}$. longum.

Frutex, ramis subteretibus striatis. Ramuli hornotini tetragoni plus minusve pubescentes circ. $1 \mathrm{~mm}$. crassi, internodiis $1-1.5 \mathrm{~cm}$. longis. Folia ovato-lanceolata vel lanceolata rarius oblonga brevissime petiolata, tenuiter coriacea, plerumque $3-3.5 \mathrm{~cm}$. longa, $0.6-$ $1.5 \mathrm{~cm}$. lata, apice emarginata vel obtuse apiculata, basi cuneata vel acuta, margine leviter deorsum recurvata, supra nitidula subtus pallidiora, costa media utrinque elevata supra in parte inferiore plus minusve puberula, nervis lateralibus utraque facie conspicuis saepissime apice bifurcatis; petioli circ. $1 \mathrm{~mm}$. longi sparse pilosi. Racemi axillares laxiflores, rhachi dense pilosa plus minusve elongata circ. $3 \mathrm{~mm}$. longa, bractearum paria 4 , bracteae ovatae vel ovato-oblongae chartaceae apice acutae convexae dorso sparse pubescentes circ. 2-2.5 mm. longae, margine vix scariosae; flores of $6-8$, pedicellati, pedicelli circ. $1.5-1.7 \mathrm{~mm}$. longi sparse pilosi, sepala orbicularia vel ovato-rotundata convexa dorso ad costam pubescentia circ. $2.5 \mathrm{~mm}$. Ionga, stamina sepala superantia circ. $4 \mathrm{~mm}$. longa, antheris circ. $1.2 \mathrm{~mm}$. longis, ovarii rudimentum sepalis circ. duplo 
breviore apice dilatato-tetragonum circ. $1 \mathrm{~mm}$. longum; flos 우 terminalis, sepala ovata convexa dorso sparse pilosa circ. $2.5-3 \mathrm{~mm}$. longa, ovarium stylis complanatis apicem versus vix angustatis leviter recurvatis duplo breviore, stigmata obcordata usque ad medium stylum decurrentia medio sulcata. Capsula ignota. Fig. 9 a-f, Pl. X, Fig. 2.

Formosa. Syusyu Nantô, May 1912 (HAYATA et SASAKI, Herb. Taihoku, no. 15100; type, in HT). Ahi-sen, Central Range, alt. 1500 m., Jan. 1, 1908 (T. KaWAKAmI et SASAKI, Herb. Taihoku, no. 15101 , in HT).

This variety is well distinguished from the type, by the characters described above which has usually much larger, somewhat thicker coriaceous leaves with fewer lateral nerves usually less conspicuous on both surfaces.

15. Buxus mollicula W. W. Smith in Not. Roy. Bot. Gard. Edinburgh, 10: 16 (1917) (Catalogue of Plantae Chinenses Forrestianae).-HaNDEL-MazzetTI, Symb. Sinc. 7: 236 (1931).

Buxus Wallichiana var. velutina FRANCHET, Pl. Delav. 136 (1889), syn. nov., secundum REHDER.

Frutex circ. $3 \mathrm{~m}$. altus, ramis subteretibus albo-cinerascentibus decorticantibus, ramuli hornotini compresso-tetragoni haud alati molliter pubescentes circ. $2 \mathrm{~mm}$. diametro. Folia coriacea breviter petiolata, oblonga vel elliptica rarius oblongo-lanceolata plerumque $4 \perp 5 \mathrm{~cm}$. longa, $1.3-2 \mathrm{~cm}$. lata, apice acuto-obtusa, emarginata vel obtusa, basi acuta vel late cuneata, margine leviter recurvata, supra primo dense adpresse puberula mox subglabra nitidula vel opaca plus minusve glaucescentia, subtus paullo pallidiora opaca molliter puberula, costa media utrinque leviter elevata dense puberula, nervis lateralibus utrinque haud vel vix conspicuis; petioli circ. $1 \mathrm{~mm}$. longi molliter puberuli. Inflorescentiae axillares ambitu breviter cylindricae, rhachi plus minusve elongata $3-4 \mathrm{~mm}$. longa dense puberula, bractearum paria circ. 6 , bracteae ovato-triangulares acutae convexae dorso dense pilosulae rubescentes circ. $1.5 \mathrm{~mm}$. longae vix scariosae; flores $\hat{o}$ sessiles (?), sepala orbicularia circ. 2 $\mathrm{mm}$. longa convexa dorso sparse pilosula rubescentia, stamina ......, ovarii rudimentum ......, flos o terminalis ignotis, sepala (sub fructu) exteriora ovato-triangularia circ. $4 \mathrm{~mm}$. longa dorso glabra, interiora quam exteriora paullo angustiora circ. $3 \mathrm{~mm}$. longa. 
angustiora circ. $3 \mathrm{~mm}$. longa. Capsula globosa circ. $1 \mathrm{~cm}$. longa in sicco olivacea nitidula, stylis persistentibus $2-3 \mathrm{~mm}$. longis apice valde recurvatis, stigmatibus usque ad $2 / 3$ stylum decurrentibus anguste obcordatis, semina trigono-elliptica circ $5 \mathrm{~mm}$. longa nitida nigerrima. Fig. 10.

China. Yunnan: without precise locality, June 22, 1888 (*Delavay, Plate no. 1601; type of $B$. Wallichina var. velutina FrANCH., fragment seen, in HA); dry, open slopes amongst scrub in the Yantze valley and on the hills around Yungpeh, Lat. $26^{\circ} 48^{\prime} \mathrm{N}$., Long. $100^{\circ} 42^{\prime}$ E., alt. 6-8000 ft., Sept. 1921 (FORREST, no. 20590, in $\mathrm{HA}$ ); mts. in the N. S. of the Yangtze bend, Lat. $27^{\circ} 45^{\prime} \mathrm{N}$, alt. $10000 \mathrm{ft}$., July 1913 (ForRest, no. 10684, in HA). S.W. Szech'uan: East of Yungnung, Lat. $27^{\circ} 50^{\prime} \mathrm{N}$, Long. $100^{\circ} 56^{\prime} \mathrm{E}$, alt. $9000-10000 \mathrm{ft}$., Oct. 1921 (ForREST, no. 20632, in HA; open thickets and forests; shrub of $6-10 \mathrm{ft}$.).

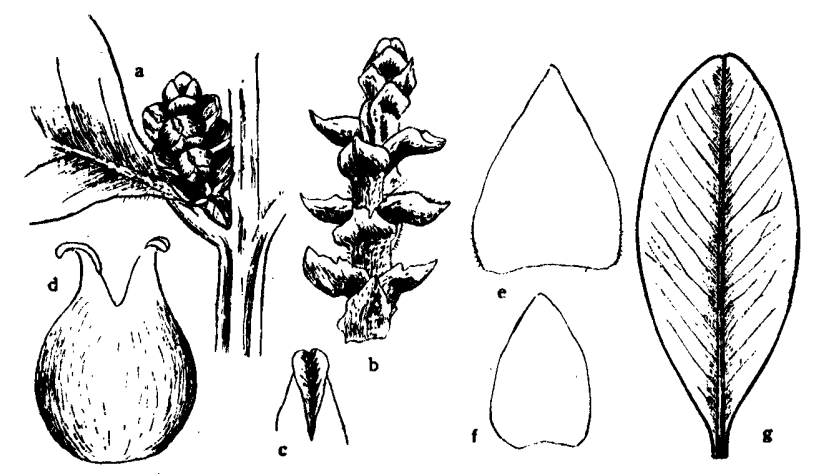

Fig. 10. Buxus mollicula Smrth (c-g), var. glabra Handed-Maz. $(\mathrm{a}-\mathrm{b})$. a, brachlet beàring undeveloped inflorescence $\times 2.5$. $b$, inflorescence, male flowers taken off $\times 5$. c, stigma mag. nified. $d$, valve of capsule, seen from without $\times 2.5$. e, $f$, inner and outer sepals of female flower, seen from within $\times 5$. $\mathrm{g}$, leaf, seen from above $\times 1$.

In its floral structure this is most closely related to B. megistophylla LÉvL. with which it agrees in the rubescent colour of the small sepals and bracts and in the elongate inflorescence. I believe

*) Of Delavay's collections Bretshneider writes (Hist. Europ. Bot. Disc. China, 875,1898 " Delavay gathered plants on the whole journey through Hupeh, Szech'uan and Yünnan, but the chief field of this exploration lay among the elevated mountains, west, north and north-east of the great lake near Talifu where he botanized for nearly ten years in different seasons." 
that these two species may represent a peculiar subgroup. For further notes see my remarks under $B$. megistophylla LÉVL. Though the fully developed male flowers of this species are not yet known, so far as I can judge, from the analysis of the young flowers before me, they seem to have sessile male flowers with rudimental ovaries apparently shorter than the sepal.

var. glabra HandeL-MAzzetTI, Symb. Sinic. 7: 236 (1931).

A typo foliis utrinque ramulisque fere glabris differt.

China. N.W. Yunnan: in regionis subtropicae faucium fluvii Djinsha-djiang (,Yangtse“) ad septentriones urbis Likiang, 27 $47^{\prime}$, alt. 2100 m., Aug. 10, 1915 (HANDEL-MAzzetTI, no. 7581; isotype in HA).

This is an extreme glabrous form of the type which exhibits considerable variation in the pubescence of the leaves and branchlets.

\section{Group.}

Ramuli hornotini subteretes glaberrimi; folia ovato-lanceolata apice acutiuscula, costa media utrinque elevata, nervis lateralibus utrinque distinctis ; racemi dense glomerati, rhachi brevissima; flores o magni longipedicellati, ovarii rudimentum gracile apice vix dilatatum sepalis oblongis membranaceis scariosissimis multo breviore, bracteae ovato-oblongae apice obtusae membranaceae scariosissimae; flos $\&$ terminalis, ovarium stylis complanatis apicem versus sensim angustatis multo breviore, stigmata lineari-obcordata fere usque ad basim stylum decurrentia. Species 1, Chinae mediae incola.

This group is most closely related to the group 2 , from which it differs in its very short inflorescences, its larger male flowers with longer pedicels, its narrower thinly membranaceous and transparent sepals and bracts, and its slender rudimental ovaries.

16. Buxus Henryi Mayr, Fremd. Waldb. u. Parkb. 451 (1906). -DÜMMER in Gard. Chron. ser. 3, 52: 423, fig. 182 (1912).-REHDER et Wilson in SARgent, Pl. Wills. 2: 168 (1914).

Frutex glaber, ramis teretibus, ramuli hornotini compresse teretes haud angulati glabri circ. $2 \mathrm{~mm}$. crassi, internodiis $1.5-3 \mathrm{~cm}$. longis. Folia brevissime petiolata, subcoriacea, late lanceolata vel 
ovato-oblonga $6-11 \mathrm{~cm}$. longa $1.5-3 \mathrm{~cm}$. lata (plerumque $2 \mathrm{~cm}$.), apice acutiuscula rarius caudato-acuminata, basi cuneata vel anguste cuneata, margine deorsum leviter recurvata, costa media utrinque distincte elevata, supra nitidula subtus pallidiora, nervis lateralibus numerosis utrinque subdistinctis. Racemi axillares densissime

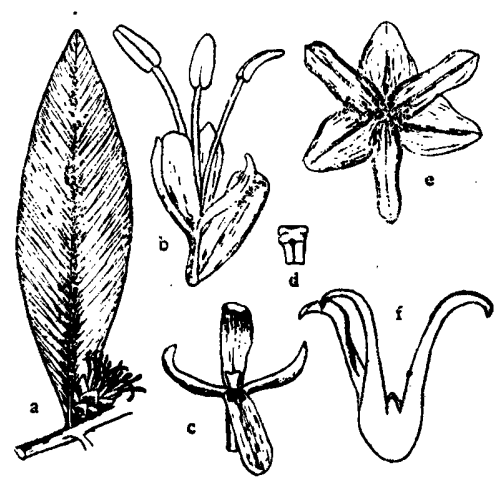

Fig. 11. Buxus Henryi Mayr (Henry, no. 3387, type). a, branchlet with inflorescence $\times 1 / 2$. b, male flower with bract $x 2$. c, same, showing rudimental ovary $\times 2$. d, rudimental ovary, seen from side $\times 4.5$. e. sepals of female flower, seen from without $\times 1.5$. $f$, ovary $\times 2$. glomerati, rhachi brevissima circ. $2 \mathrm{~mm}$. longa glabra, bractearum paria plerumque 6 , bracteae basilares ovatae acutae cinereo-fuscescentes chartaceae, margine late scariosae $3-5 \mathrm{~mm}$. longae, superiores obovato-oblongae circ. 6 $\mathrm{mm}$. longae $2.5 \mathrm{~mm}$. latae membranaceae scariosissimae; flores $\hat{o}$ longe pedicellati, pedicelli $2-4 \mathrm{~mm}$. longi glabri, sepala oblonga vel obovato-oblonga $4.5-5 \mathrm{~mm}$. longa 2 $\mathrm{mm}$. lata glabra membranacea scariosissima, stamina sepala superantia circ. $9 \mathrm{~mm}$. longa, antheris 2 $\mathrm{mm}$. longis, ovarii rudimentum gracile sepalis $3-4$ plo breviore apice vix dilatatum circ. $1-1.5 \mathrm{~mm}$. longum; flos o terminalis, sepala

3 exteriora oblonga circ. $6 \mathrm{~mm}$. longa glabra scariosa, 3 interiora quam exteriora latiora ovata circ. $3 \mathrm{~mm}$. longa glabra scariosa, ovarium stylis complanatis apicem versus sensim angustatis recurvatis circ. 3 plo breviore (ovarium $2-2.5 \mathrm{~mm}$. longum, styli $6-8 \mathrm{~mm}$. longi), stigmata lineari-obcordata fere usque ad basim stylum decurrentia. Capsula ignota. Fig. 11.

China. W. Hupeh: Ichang and immediate neighbourhood (A. HENRY, no. 3387, in HA). Kweichow: Pingchow, Sept. 14, 1930 (Y. TSIANG, no. 7121, in HSa).

In its foliage and rounded branchlets this interesting box resembles $B$. megistopylla LÉvL. which has quite different inflorescences with much smaller male flowers. This is somewhat allied also to $B$. hainanensis MERR., but easily ditinguished from it by its almost rounded branchlets, and by its very short inflorescences with larger male flowers. 


\section{Group.}

Folia anguste oblonga glabra glaucescentia utrinque opaca subtus dense papillosa, costa media in sicco supra subplana vel leviter impressa subtus leviter elevata, nervis lateralibus utrinque vix conspicuis; inflorescentiae dense glomeratae, rhachi brevissima glabra; flores $\hat{o}$ sessiles, ovarii rudimentum gracile apice vix dilatatum sepalis 2-3 plo breviore; flos + terminalis, ovarium stylis crassis apicem versus leviter angustatis et recurvatis paullo longiore, stigmata anguste obcordata usque ad medium stylum decurrentia; capsula opaca glabra. Species 1, Himalayae incola.

This interesting group does not seem to be closely related to any other groups. In its dull glaucous leaves with the midribs usually scarcely elevated or slightly impressed above it agrees with the group 1, but differs from it in the floral structure.

17. Buxus papillosa SchneIder, Illus. Handb. Laubholzk. 2: 139, fig. $89 \mathrm{v} ; 90 \mathrm{k}-1 .-$ ReHDer et Wilson in SARgENT, Pl. Wils. 2: 168 (1914).

Frutex glaucus glaber, ramis teretibus, ramuli compresse tetragoni circ. 1-2 mm. diametro. Folia lineari-oblonga, anguste oblanceolata vel oblonga rarius elliptica $2-7 \mathrm{~cm}$. longa (plerumque 4-6 cm.) $0.4-1.2 \mathrm{~cm}$. lata (plerumque $0.5-0.8 \mathrm{~cm}$.), coriacea vel tenuiter coriacea, apice acutiuscula rarius obtusa vel brevissime emarginata, basi sensim angustata, margine haud recurvata, utraque facie glaucescentia supra opaca subtus pallidiora dense papillosa, costa media supra vix elevata vel leviter impressa subtus leviter elevata, nervis lateralibus utrinque vix conspicuis, petioli circ. $1 \mathrm{~mm}$. longi glabri. Inflorescentiae axillares dense glomeratae, rhachi glabra brevissima circ. $3 \mathrm{~mm}$. longa, bractearum paria 4 vel 5 , bracteae ovatae acutae flavescentes chartaceae 2-2.5 mm. longae glabrae; flores 1 . sessiles, sepala ovato-elliptica, $2.5-3 \mathrm{~mm}$. longa, membranacea scariosa, stamina sepala superantia $5-7 \mathrm{~mm}$. longa, antheris $1.5-2$ $\mathrm{mm}$. longis, ovarii rudimentum gracile sepalis 2-3 plo breviore apice vix dilatatum; flos i terminalis, sepala ovata convexa membranacea apice obtusa margine late scariosa dorso glabra 3-3.5 mm. longa, ovarium stylis apicem versus sensim leviter angustatis et recurvatis paullo longiore (ovarium $4-5 \mathrm{~mm}$. longum, styli $3 \mathrm{~mm}$. longi), stigmata anguste obcordata usque ad $2 / 3$ stylum decurrentia. Capsula ovoidea laeviuscula vel opaca cum stylis persistentibus crassis, 


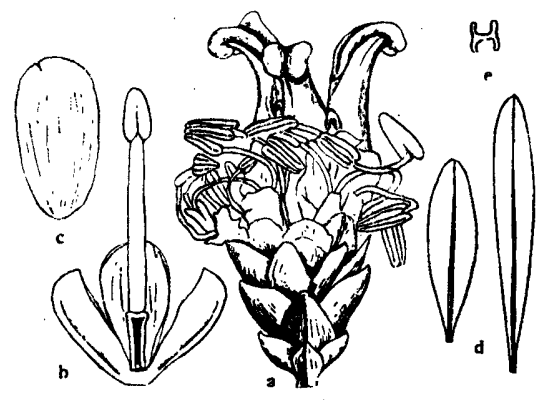

Fig. 12. Buxus papillosa Schnemer. a, inflorescence $\times 5$. $\quad b$, male flower, inner sepal and three stamens taken off $\times 5$. c, inner sepal of the same $\times 5$. d, leaves of different shapes $1 / 2$. e, rudimental ovary, seen from above $\times 3.5$.

lanceolatis rigidis apice recurvatis $2-2.5 \mathrm{~mm}$. longis circ. $1 \mathrm{~cm}$. longa; semina trigono-ellipsoidea circ. $5 \mathrm{~mm}$. longa nigerrima lucida. Fig. 12.

India. N.W. Himalaya: Punjab: Camp Sodi, Salt Range, May 14, 1928 (BIS RAM., no. 830, in HA ; vern. name, Papar or Chikari); Sidapur, Rawalpindi, alt. $1800 \mathrm{ft.}$, Nov. 8, 1924 (R. R. STEWART, no. 1588, in HA); Chattar, Murree boad, alt. $2000 \mathrm{ft}$., April 21, 1917 (STEWAR'T, no. 1662, in HA); Rawalpindi, alt. $1700 \mathrm{ft}$., March 8, 1922 (STEWART, without number, in HA; cultivated).

W. Himalaya: . Moorga, Baluch, May 23, 1917 (Duthie, no. 19009 , in HA).

In its slender rudimental ovaries and transparent sepals of the male flowers in the densely glomerate inflorescences, this box resembles $B$. Henryi MAYR, from which it differs in the smaller sessile male flowers, in the shorter styles of the ovary, and in the narrower glaucous leaves densely papillous beneath, etc.

\section{Group.}

Ramuli hornotini tetragoni plus minusve pubescentes; folia coriacea vel tenuiter coriacea, supra viridia lucida rarius opaca glaucescentia, 'costa media supra valde elevata subtus obtuse elevata, nervis lateralibus supra distinctis subtus obsoletis. Inflores- 
centiae dense glomeratae, rhachi brevissima; flores $\hat{o}$ sessiles vel breviter pedicellati, ovarii rudimentum sepalis circ. 2-3 plo breviore apice valde dilatatum; flos o terminalis, ovarium stylis crassis brevis apicem versus sensim dilatatis et recurvatis longiore, stigmata obcordata usque ad medium stylum decurrentia. Capsula nitidula.

Species 3, Chinae occidentalis et Himalayae incolae.

This group is most closely related to the group 7, from which it differs in the shorter rudimental ovaries, and in the stalked male flowers (excl. B. Wallichiana BAILLoN).

18. Buxus Wallichiana BaIllon, Monogr. Buxac. 63 (1859). --Mueller-Arg. in De Candolle, Prodr. 16: 18 (1869).-Koehne, Deutsch. Dendrol. 356 (1893):-Elwes \& Henry, Trees Gt. Brit. Irel. 7: 1723 (1913) pro parte, excl. syn.-SchNeidER, Illus. Handb. Laubholzk. 2: 139, fig. $90 \mathrm{~h}-\mathrm{i}$ (1907).-REHDER et WILSON in SARGENT, Pl. Wils. 2: 168 (1914).

Buxus sempervirens sensu BRANDIs, Forest Fl. Brit. Ind. 447 (1874); Indian Trees ed. 4, 559 (1921), non LinNAEUs.-J. D. HooKER, Fl. Brit. Ind. 5: 267 (1887).-GambLE, Indian Timbèrs, 592 (1902).

Buxus longifolia sensu Balllon, Monogr. Buxac. 63 (1869) quasi synon., non BoIssIER.

Frutex ad $6 \mathrm{~m}$. altus, rami cinerascentes longitudinaliter irregulariter striati subteretes, ramuli hornotini tetragoni circ. $1.5 \mathrm{~mm}$. crassi dense pubescentes rarius glabrescentes, internodiis plerumque $1-1.5 \mathrm{~cm}$. longis. Folia breviter petiolata, coriacea vel tenuiter coriacea, lanceolata, oblongo-lanceolata vel ovato-lanceolata, plerumque $4-5 \mathrm{~cm}$. longa, 7-10 mm. lata, apice obuse acuta vel acutiuscula, basi sensim angustata vel acuta, margine deorsum leviter recurvata, supra viridia nitidula glabra subtus pallidiora glabra, costa media utrinque valde elevata supra in parte inferiore plerumque pubescentia subtus glabra, nervis lateralibus numerosis mediis angulo $40^{\circ}$ a costa egressis supra distinctis subtus obsoletis ; petioli $1.5-2$ $\mathrm{mm}$. longi dense pubescentes. Inflorescentiae axillares dense glomeratae ambitu globosae, rhachi brevissima circ. $3 \mathrm{~mm}$. longa dense pubescentia; bracteae ovato-rotundatae chartaceae canvexae dorso ad costam sparse pubescentes circ. $4 \mathrm{~mm}$. longae margine anguste rubescentes; flores $\hat{\delta}$ sessiles, sepala late ovata scariosa dorso ad costam sparse pilosa circ. $3 \mathrm{~mm}$. longa, stamina sepala superantia circ. $5 \mathrm{~mm}$. longa, antheris circ. $2 \mathrm{~mm}$. longa, ovarii rudimentum 
sepalis 2-3 plo breviore apice valde dilatatum circ. 1-2 mm. longum; flos + terminalis, sepala late ovata, apice obtuse acuta, convexa membranacea scariosa dorso glabra circ. $2.5 \mathrm{~mm}$. longa, ovarium stylis crassis apicem versus sensim dilatatis leviter recurvatis circ. 1.5 plo longiore, stigmata obcordata vel anguste obcordata usque ad medium vel $2 / 3$ stylum decurrentia. Capsula ovoidea laeviuscula cum stylis persistentibus rigidis circ. $3 \mathrm{~mm}$. longis circ. $8 \mathrm{~mm}$. longa ; semina trigonoellipsoidea circ. $6 \mathrm{~mm}$. longa $3 \mathrm{~mm}$. lata nigerrima lucida. Fig. 13.

India. N.W.Himalaya; Chakrata, Siwalik \& Jaunsar, alt. $7000 \mathrm{ft}$., May 14, 1912 (SUlaKHAN, no. 86; vern. name: Chikri, in HA); same locality, alt. 6900 ft., April 9, 1900 (Hudson, no. 81 , in HS); Ialla, Kali valley, Almora District, alt. $2560 \mathrm{~m}$., July 11 , 1923 (PARKER, no. 2070, in HA); Kainthli Reserve, Perganna Chuari, Chamba State, alt. 2000 m., April 6, 1920 (PARKER, without number, in $\mathrm{HA}$; without precise locality, alt. $6000 \mathrm{ft}$., 1886 (H. MayR; small tree, in HA); Mandi, Poonch, Kasimir, alt. 4000 m., April 1931 (STEWART, no. 19089, in HA). W. Himalaya: Dorkali, Rampur-Bushahr, Punjab, alt. 2000 m., Nov. 28, 1931 (Walter Koelz, no. 3214 ; shruby tree $20 \mathrm{ft}$. tall; fruit bluish; bark pale gray,

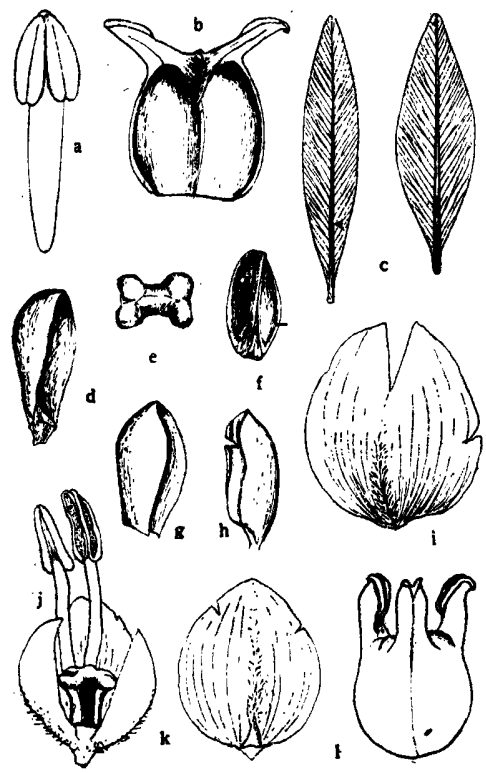

Fig. 13. Buxus Wallichiana Ballion. a, stamen $\times 5$. b, valve of capsule, from seen within $\times 2$. c, leaves of different shapes $\times 1 / 2$. d, external sepal of male flower, seen from side $\times 5$. e, rudimental ovary, seen from above $\times 5$. $f$, seed $\times 2$. $g, h$, inner and outer sepals of female flower, seen from side $\times 5$. i, bract, seen from without $\times 5$. j, male flower, inner sepal and two stamens taken off $\times 5$. $k$, inner sepal of same, seen from without $\times 5$. 1, ovary $\times 3$. scaly; in HA).

This species is apparently closely related to $B$. rugulosa HATUSIMA from which it is easily distinguished by its larger and narrower leaves with the distinctly elevated midrib beneath and much prominent lateral nerves on the less rugose upper surface, and by its sessile male flowers. GaGnePaIN (Lecomte, Fl. Gén. Indo- 
Chine 5: 662, 1927) admits this box from Tonkin and China, but I have seen no material from China referable to this species, and it seems to be very doubtful if the true $B$. Wallichina BILlon occurs in China or Tonkin. GagnePAIN probably included $B$. ruglosa HATUSIMA in his concept of the Himalayan species.

19. Buxus hebecarpa Hatusima, sp. nov.

Frutex ramulis tetragonis dense breviter pilosis circ. $2.5-3 \mathrm{~mm}$. crassis, internodiis $1.5-2.5 \mathrm{~cm}$. longis. Folia crasse coriacea breviter petiolata, ovato-lanceolata vel lanceolata rarius ovata, $4.5-6 \mathrm{~cm}$. longa $1.5-2 \mathrm{~cm}$. lata, apice acutiuscula, margine deorsum revoluta, basi cuneata, supra nitidula subtus opaca, costa media supra valde elevata breviter pilosa subtus leviter elevata glabra, nervis lateralibus supra distinctis subtus obsoletis; petioli $2-3 \mathrm{~mm}$. longi, supra sulcati dense pilosi. Inflorescentiae axillares dense glomeratae, rhachi dense pilosa circ. $7 \mathrm{~mm}$. longa, bractearum paria 3 , bracteae ovatae acutae concavae circ. 3

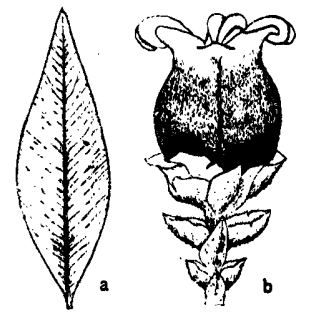

Fig. 14. Buxus hebecarpa Hitusims. a, leaf, seen from surface $\times 1 / 2$. $b$, inflorescence, bearing capsule $\times 2.5$. $\mathrm{mm}$. longae dorso dense pilosae, flores $\hat{o}$ ignoti; flos $q$ terminalis ignotis, sepala (sub fructu) interiora fere orbicularis margine late scariosa circ. $4 \mathrm{~mm}$. longa dorso sparse pilosa, exteriora crassiora leviter angustiora apice obtuse acuta dorso dense pilosa, circ. $3 \mathrm{~mm}$. longa. Capsula globoso-ovoidea circ. $9 \mathrm{~mm}$. longa, dense tomentella, stylis persistentibus apice valde recurvatis circ. $3 \mathrm{~mm}$. longis, stigmatibus usque ad basim stylum decurrentibus obcordatis; semina trigono-ellipsoidea circ. $5 \mathrm{~mm}$. longa nigra. Fig. 14.

China. Szech'uan: Omei-hsien (T. TU, no. 239, Szech'uan Expedition of Research Institute of western China, 1935; type in HSa); ? without precise locality and date (W. FANG, no. 8597; an undeveloped flowering specimen; in HSa).

In the foliage this new box is most closely related to $B$. microphylla subsp. sinica HATUSIMA, but it is easily distinguished from it by its densely pubescent branchlets with much larger leaves and by its densely pubescent fruits with strongly revolute styles.

FANG's no. 8597, a specimen doubtfully cited above, has undeveloped flowers with sessile 'male flowers with sepals and bracts 
densely pubescent on the dorsal side. The leaves are somewhat smaller than those of the type.

\section{Buxus rugulosa Hatusima, sp. nov.}

Buxus microphylla var. sinica REHDER et WILSON in SARGENT, Pl. Wils: 2: 165 (1914), pro parte, quoad pl. ex Yunnan.

? Buxus Wallichiana sensu Gagnepain in Lecomte, Fl. Gén. Indo-Chine 5: 662 (1927), excl. syn.-non BaIlLoN.

Buxus microphylla var. platyphylla HANDEL-MAzzETTI, Symb. Sinic. 7: 237 (1931), excl. syn.

Frutex plus minusve prostratus (semper?), ramis subteretibus albo-cinerascentibus, ramuli tetragoni pubescentes circ. 1-2 mm. crassi, internodiis plerumque $1 \mathrm{~cm}$. longis. Folia breviter petiolata, coriacea, rhombeo oblonga vel elliptica vel oblonga rarius lanceolata vel ovata, $2-3 \mathrm{~cm}$. longa $0.7-1.2 \mathrm{~cm}$. lata, apice emarginata vel obtusa rarius acutiuscula, basi sensim angustata vel cuneata, margine revoluta, supra viridia, nitidula in sicco valde rugulosa, subtus opaca flavescentia, costa media supra valde elevata in parte' inferiore plerumque pilosa, subtus leviter. elevata glabra, nervis lateralibus supra vix conspicuis subtus obsoletis; petioli 2-3 mm. longi dense pilosi. Racemi axillares dense glomerati globosi, rhachi brevissima circ. 3-4 $\mathrm{mm}$. longa dense pilosa, bractearum paria $6-8$, bracteae ovatae acutiusculae convexae pallide fuscescentes, margine late scariosae ad $3 \mathrm{~mm}$. longae dorso ad costam mediam sparse pilosae; flores $\hat{o}$ distincte breviter pedicellati (circ. $0.5-1 \mathrm{~mm}$. longi), sepala late ovata convexa pallide fuscescentia circ. $3 \mathrm{~mm}$. longa dorso glabra, stamina ignota, ovarii rudimentum sepalis $1.5-2$ plo breviore circ. 1-1.5 mm. longum apice valde dilatatum capitatotruncatum, 4-sulcatum; flos 9 terminalis, sepala late ovata acutiuscula circ. $3 \mathrm{~mm}$. longa dorso sparse pilosa. Capsula cum stylis persistentibus crassis circ. $3 \mathrm{~mm}$. longis divaricatis usque ad $1.2 \mathrm{~cm}$. longa, glabra laeviuscula, stigmata obcordata usque ad medium stylum decurrentia; semina trigono-ellipsoidea $6-7 \mathrm{~mm}$. longa nigerrima lucida. Fig. 15a-b, Pl. VII, fig. 2.

China. Yunnan: in silvis in grosser Dlinc ad pedem mt. nivecorum prope Lichiang, alt. $3000 \mathrm{~m}$., Oct. 1914 (C. SCHNEIDER, no. 3285 , in HA ; shrub 1-2 m. ht., type); same locality, alt. $2900 \mathrm{~m}$., Sept. 4, 1914 (C. SchneInER, no. 3015, in HA); Yangtze wațershed, 
Prefectural District of Likiang, eastern slopes of Likiang Snow Range, May-Oct. 1922 (Rock, no. 4734, in HA; shrub, more or less prostrate); prope urbem Likiang, imprimis in monte Yülung-schan, June-Sept. 1914-1918 (HANDEL-MAZZETTI, no. 3765, in HA); Mekong watershed, en route to Youngchang and Tengyueh, Sept.-Oct. 1922 (Rock, no. 6793, in HA); Mentz, north mts., in forest, alt. $8500 \mathrm{ft}$. (HENRY, po. 11157, in HA; tree $10 \mathrm{ft}$. ht.). N.W. Szech'uan: Ch'osodjo, Oct. 22, 1918 (H. SMITH, no. $4651_{2}$ in HA).

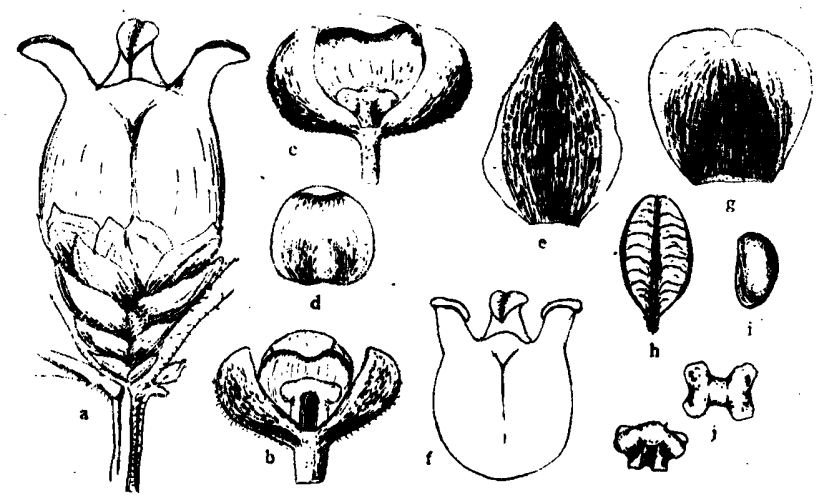

Fig. 15. Buxus rugulosa Hatusim (a-b), var. intermedia $\mathrm{H} \Lambda \mathrm{TU}$ sMA (c-j; Rock, no. 17301). a, branchlet with infructescence bearing capsule $\times 4$. b, male flower, inner sepal and stamens taken off $\times 5$. c, same, showing rudimental ovary $\times 5$. d, inner sepal of same, seen from within $\times 5$. e, bract, seen from without $\times 5$. $f$, capsule $\times 2$. $g$, sepal of female flower, seen from within $\times 5$. h, leaf, seen from surface $\times 1$. i, seed $\times 1.5$. $j$, ovaries rudimental, seen from above and side $\times 5$.

This species has been confused with B. microphylla subsp. sinica HATUSIMA and naturally enough, as the foliage of the two species is very similar. But, with the complete material before me it becomes obvious that they are quite distinct, if closely related, species.

The Yunnan species has generally narrower rugose leaves with less prominent lateral nerves above, and shortly stalked male flowers with subsessile rudimental ovaries. It exhibits considerable variation in the size and shape of the leaves, as in the 
pubescence of the branchlets. HENRY, no. 11157 has larger ovate leaves up to $3.5 \mathrm{~cm}$. long.

The reddish brown colour of the sepals and bracts of this species together with its following subspecies seems to have a taxonomic value. For further note, see under $B$. Wallichiana BAILLON.

var. intermedia Hatusima, var. nov.

A typo recedit ramis ramulisque densioribus, foliis minoribus vix ultra $1 \mathrm{~cm}$. longis, subtus primo plus minusve papillosis. A $B$. ruguloso subsp. prostrato recedit ramulis crassioribus dense tomentellis, foliis majoribus costa media dense pubescentia, flores masculis paullo majoribus, bracteae sepalaque rubescentes, capsula majora.

Frutex circ. $1 \mathrm{~m}$. altus, caulis erectus (semper?), cortice albocinerascente decorticante, rami subteretes pilis nigrescentibus dense obtecti, ramuli luteo-tomentelli circ. 1-2 mm. crassi tetragoni, internodiis circ. $5 \mathrm{~mm}$. longis. Folia ovata vel ovato-elliptica rarius ovato-oblonga breviter petiolata, crasse coriacea, apice obtuse acuta vel obtusa, basi acuta vel cunneata, margine valde revoluta, supra nitidula valde rugulosa, subtus pallidiora flavescentia, costa media supra valde elevata in parte inferiore dense pilosa, subtus leviter elevata glabra, nervis lateralibus utrinque obsoletis; petioli circ. $2 \mathrm{~mm}$. longi dense pubescentes. Racemi axillares dense glomerati globosi, rhachi brevissima dense pilosa circ. $3 \mathrm{~mm}$. longa, bractearum paria circ. 6 , bracteae ovatae acutae convexae pallide rubescentes, dorso basim versus pilosulae, margine late scariosae ciliolatae circ. $4 \mathrm{~mm}$. longae; flores $\hat{o}$ breviter pedicellati (1-1.2 $\mathrm{mm}$. longi), sepala ovato-rotundata apice obtuse acuta, convexa apice incurva dorso dense rubescento-striata glabra circ. 2-2.5 mm. longa, stamina ignota, ovarii rudimentum sepalis $2-3$ plo breviore apice dilatatum tetragono-truncato-capitatum 4-sulcatum; flos $O$ terminalis, sepala ovato-rotundata apice laciniato-obtusa dorso pallide rubescentostriata glabra circ. $3 \mathrm{~mm}$. longa scariosa. Capsula cum stylis persistentibus crassis circ. $3 \mathrm{~mm}$. longis divaricatis usque ad $1 \mathrm{~cm}$. longa, stigmata obcordata usque ad medium stylum decurrentia. Fig. $15 \mathrm{c}-\mathrm{j}$, Pl. VIII, Fig. 1.

China. N.W. Yunnan: Mount Schwe-Men-Kai, southeast of Chung-tien, alt. 3550 m., Aug.-Sept. 1928 (Rock, no. 17301, in HA; shrub $1 \mathrm{~m}$. ht.; type). 
In its foliage this variety is an intermediate form between the type and its subsp. prostrata HATUSIMA, and connected by intermediate form with both species. This is also closely connected with $B$. rugulosa subsp. rupicola HATUSIMA which has non-shining glaucous leaves densely pubescent on both surfaces, and pubescent ovaries.

subsp. prostrata (SMith) Hatusima, comb. nov.

Buxus sempervirens var. microphylla sensu HOOKER, Fl. Brit. Ind. 5 : 267 (1887).-Brandis, Ind. Trees ed. 4, (1921).-non MUeller-ARg.

Buxus microphylla var. prostrata W. W. SMITH in Not. Roy Bot. Gard. Edinb. 10: 16 (1917).-Handel-MazzetTI, Symb. Sinic. 7: 237 (1931).

Frutex humilis prostratus ramosissimus, cortice cinerascente suberoso longitudinaliter fisso, ramis subteretibus parce pilosulis vel fere glabris saepe radicantibus, ramuli tetragoni parce pilosuli vel fere glabri circ. $1 \mathrm{~mm}$. crassi, internodiis $2-5 \mathrm{~mm}$. longis. Folia breviter petiolata, petioli circ. $1 \mathrm{~mm}$. longi supra plani parce pilosi vel glabri, coriacea, ovato-elliptica, ovata vel obovata, apice emarginata vel obtusa, basi acuta, margine valde revoluta 5-9 $\mathrm{mm}$. longa 3-6 mm. lata, supra nitidula rugulosa subtus flavescentia, costa media supra elevata glabra, nervis lateralibus supra haud subtus vix conspicuis, Racemi axillares et terminales dense glomerati globosi, rhachi brevissima parce pilosa circ. $2 \mathrm{~mm}$. longa, bractearum paria $4-5$, bracteae late ovatae vel orbiculares obtusae circ. $2 \mathrm{~mm}$. longae pallide fuscescentes vel lutescentes (sub plantae himalayenses) margine scariosae ciliatae; flores $\hat{\alpha}$ breviter pedicellati (0.5-0.8 mm. longi), sepala ovato-elliptica glabra circ. $2 \mathrm{~mm}$. longa, stamina sepala superantia circ. $2.5 \mathrm{~mm}$. longa, antheris circ. $1 \mathrm{~mm}$. longis, ovarii rudimentum sepalis $2-2.5$ plo breviore $0.7-0.8$ $\mathrm{mm}$. longum apice valde dilatatum; flos + terminalis, ovarium stylis brevis crassis apicem versus dilatatis paullo longiore glabrum, stig. mata obcordata usque ad medium stylum decurrentia. Capsula cum stylis persistentibus lanceolatis circ. $2 \mathrm{~mm}$. longis usque ad $6 \mathrm{~mm}$. longa; semina trigono-ellipsoidea nigerrima lucida circ. 4 mm. longa. Fig. 16, Pl. IX.

China. Yunnan: Yangtze watershed, Prefectural District of Likiang, eastern slopes of Likiang Snow Range, May-Oct. 1922 
(Rock; no. 3686, in HA; shruby lrock plant); inter Yunging et Mudidjin, alt. 2400 m., Jan. 23, 1914 (C. SCHNEIDER, no. 3510, in HA) ; Likiang Range, Lat. $27^{\circ} 40^{\prime} \mathrm{N}$, alt. $3700 \mathrm{~m}$., June 1913 (FoRREST, no. 10184 , isotype in HA; prostrate shrub, 12-18 inch.); Eastern flank of Lichiang Range, on the face of limestone cliffs, Aug. 1910 (FoRREsT, no. 6300, co-type; not seen; prostrate shrub of 2 $4 \mathrm{ft}$.

S.E. Tibet: $28^{\circ} 25^{\prime}$ Lat. and $97^{\circ} 55^{\prime}$ Long., alt. $3000 \mathrm{~m}$., Sept. 11, 1931 (F. KINGDON Ward, no. 10071, in HA; on south-facing gneiss cliff above the river, where it forms low spreading bushes, more than 18 inches high.).

India. W. Himalaya: Almora, between Nabi et Kuthi, alt. 3300 m., July 16, 1923 (PARKER, no. 2091, in HA: dwarf shrub on rocks).

This alpine box differs from the type in its prostrate habit, its slender glabrescent branchlets with smaller leaves, and its somewhat smaller flowers and capsules. The specimens from the Himalayas, PARKER no. 2091, slightly differs from the Yunnan

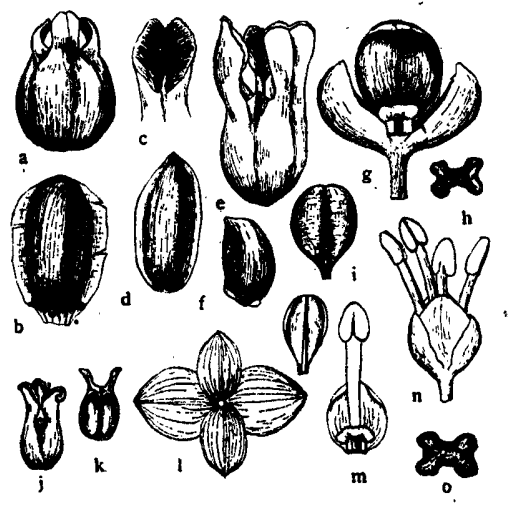

Fig. 16. Buxus rugulosa subsp. prostrata Hatusima (a-h, Parker, no. 2091 from the Himalayas; i-o, Rock, nos, 3510 , 3686 from Yunnan). a, capsule $\times 2$, $b$, bract, seen from without $\times 5$. c, stigma magnified. $d$, inner sepal of male flower, seen from without $\times 5$. e, ovary $\times 5$. f, seed $\times 2.5$. g, male flower, inner sepal and stamens taken off $\times 5$. h, rudimental ovary, seen from above $\times 5$. i, leaves of different shapes $\times 1$. $\mathrm{j}$, ovary $\times 4$. $\mathrm{k}$, valve of capsule, seen from within $\times 1$. 1, sepals of male flower, seen from without $\times 4$. $\mathrm{m}$, male flower, three stamens and sepals taken off, showing rudimental ovary $\times 4$. $n$, male flower $\times 4$. o, rudimental ovary, seen from above $\times 4$. specimens in its pale yellow colour of the bracts and sepals, its ovaries with somewhat longer styles much dilated toward the apex. The Himalayan specimens which were referred by J. D. HOOKER to B. sempervirens var. microphylla MUELLER-ARG. from Japan may probably belong here. KINGDON no. 10071 from Tibet has somewhat larger leaves and approaches somewhat $B$. rugulosa var. intermedia HATUSIMA.

subsp. rupicola (W. W. SMITH) HatUSima, comb. nov. 
Buxus microphylla var. rupicola W. W. SMITH in Not. Roy. Bot. Gard. Edinb. 9: 88 (1916).-HANDEL-MAZZETTI, Symb. Sinc. 7: 237 (1931).

Frutex circ. $1 \mathrm{~m}$. altus, ramis subteretibus pilis fusco-lutescentibus obtectis longitudinaliter fissis, ramuli tetragoni circ. $1 \mathrm{~mm}$. crassi dense fulvo-pilosulis, internodiis $5-10 \mathrm{~mm}$. longis. Folia breviter petiolata, coriacea, rhombeo-elliptica vel ovato-elliptica vel oblongo-lanceolata vel lanceolata, $1.5-2 \mathrm{~cm}$. longa et $0.5-1 \mathrm{~cm}$. lata, apice obtuse acuta rarius mucronulata vel obtusa, basi cuneata, margine leviter revoluta, in sicco supra opaca haud nitidula sparse pilosula demum costa excepta fere glabra haud rugulosa, subtus pallidiora opaca plus minusve glaucescentia primo dense pilosula demum costa excepta fere glabra, costa media utrinque leviter elevata, nervis lateralibus in sicco utrinque obscuris; petioli circ. 1-1.5 mm. longi dense fulvo-pilosi. Racemi axillares dense glomerati globosi, rhachi brevissima dense pilosa circ. $2 \mathrm{~mm}$. longa, bractearum paria 6 , bracteae ovato-triangulares apice obtuso-acutae $\cdot 2-2.5 \mathrm{~mm}$. longae dorso dense pilosulae intus parce pilosulae, margine anguste scariosae; flores $\hat{o}$ breviter distincte pedicellati (circ. $1 \mathrm{~mm}$.);

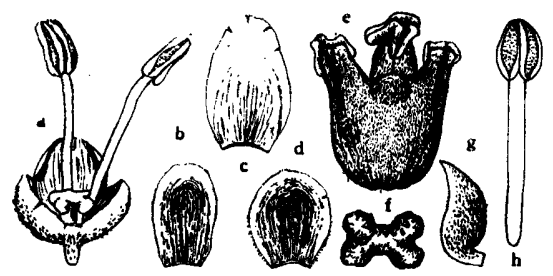

Fig. 17. Buxus rugulosa subsp. rupicola $\mathrm{HATU}$. SIMA (DeLAvay, without number). a, male flower, two stamens and inner sepal taken off $\times 4$. b, c, outer and inner sepals of female flower, seen from within $\times 4$. d, $g$, bracts, seen from within and side $\times 4$. $e$, ovary $\times 3$. $f$, rudimental ovary, seen from above $\times 5$. $h$, stamen $\times 4$. sepala ovata flavescentia margine late scariosa" et ciliata apice acutiuscula basin versus sparse pilosula circ. $2 \mathrm{~mm}$. longa, stamina sepala superantia circ. $6 \mathrm{~mm}$. longa, ovarii rudimentum sepalis $2-3$ plo breviore apice valde dilatatum; flos of terminalis, sepala exteriora elliptica convexa apice obtusa circ. $2.5 \mathrm{~mm}$. longa glabra, interiora ovata apice obtusa circ. $3 \mathrm{~mm}$. longa flavescentia membranacea margine late scariosa dorso glabra, ovarium stylis crassis brevis apice valde dilatatis paullo longiore dense pilosulum, stigmata valde dilatata obcordata media sulcata basi saepe bilobata. Fructibus immaturtis cum stylis persistentibus crassis lanceolatis circ. $3 \mathrm{~mm}$. longis usque ad $8 \mathrm{~mm}$. longis sparse pilosulis. Fig. 17, PI. VIII, Fig. 2.

China. Yunnan: without precise locality, 1888 (DelavaY, 
without number, in HA); without precise locality (FoRREST, no. 10866, in HA); on the Kari Pars, Mekong-Yangtze divide, Lat. $27^{\circ} 40^{\prime}$, alt. 12000 ft., Aug. 1914 (FoRREST, no. 13075, type, not seen; shrub of 2-3 ft.; open situation amongst rocks).

I have not seen the type of var. rupicola SMITH, but FoRREST's no. 10866 cited above agrees exactly with the original description and I have no doubt of its identity. DeLAvaY's collection, of which I have given a description above, has somewhat broader leaves than the type, but the leaf surface is dull and the veins are obscure on both surfaces as in the type. This subspecies is most closely related to $B$. rugulosa. var. intermedia, but it is readily distinguished by its less rugose leaves which are glauous and pubescent on both surfaces, and by its densely pubescent ovaries with much dilated stigmas usually clefted at the base.

\section{Group.}

Inflorescentiae racemosae vel spicatae, rhachi plerumque elongata rarius brevia; folia oblanceolata vel spathulata rarius obovata saepissime supra medium latissima, costa media in sicco subtus acute elevata (excl. no. 21), nervis lateralibus subtus plu' minusve distinctis (excl. no. 21). Capsula plerumque minora, glabra rarius tomentella. Species 5, Chinae mediae et australis incolae..

This group is characterized by its slender more or less puberulent branchlets with narrower leaves usually broadest above the middle, its lateral nerves and midribs distintly elevated beneath when dried, and by its relatively small capsules. The species belonging to this group are usually dwarf shrubs (excl. B. Bodinieri LÉvL.).

21. Buxus ichangensis Hatusima, sp. nov.

Buxus Harlandii sensu ReHder et WILSON in SARgent, Pl. Wils. 2: 166 (1914), pro parte, excl. pl. ex Hongkong.-non HANCE.

Frutex humilis circ. $0.15-1 \mathrm{~m}$. altus dense ramosus, ramuli hornotini tetragoni sparse pilosuli vel fere glabri graciles. Folia anguste obovata vel oblanceolata apice mucronulata vel rotundata rarius emarginata, basi sensim angustata, margine deorsum leviter revoluta, tenuiter coriacea $1-2 \mathrm{~cm}$. longa (plerumque circ. $1.7 \mathrm{~cm}$.) $4-6 \mathrm{~mm}$. lata, utrinque glabra, supra viridia nitidula, subtus pallidiora, costa media supra elevata in parte inferiore plus minusve puberula, subtus leviter elevata glabra, nervis lateralibus utrinque 
obscuris; petioli circ. $1 \mathrm{~mm}$. longi intus plani sparse puberuli vel fere glabri. Racemi axillares glomerati ut videtur ellipsoidei, rhachi plus minusve elongata dense pilosula $5-7 \mathrm{~mm}$. longa, brac-

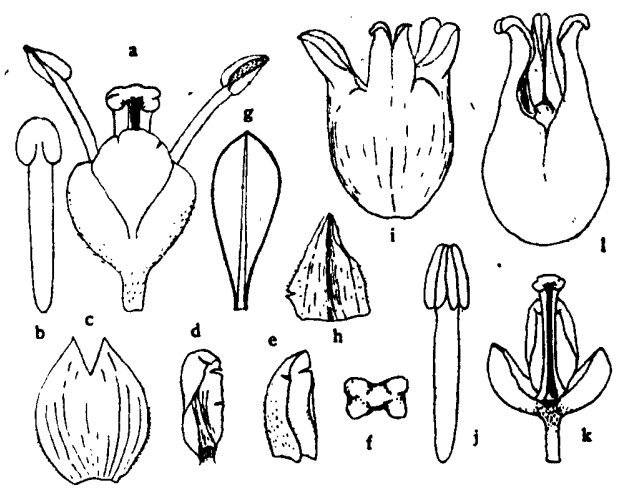

Fig. 18. Buxus ichangensis Hatusima (a-i), var. fukienensis Hatusima $(\mathrm{j}-\mathrm{k})$. a, male flower $\times 5$. b, stamen $\times 5$. c, inner sepal of male flower, seen from within $\times 5$. d, outer sepal of male flower, seen from side $\times 5$. $e, h$, bracts of different shapes, seen from side and without $\times 5$. $f$, rudimental ovary, seen from above $\times 5$. i, ovary $\times 5$. j, stamen $\times 5$. k, male flower, inner sepal and stamens taken off $\times 5$. 1, premature capsule $\times 3$.

tearum paria circ. 8 , bracteae ovatae acutae convexae 1-2 $\mathrm{mm}$. longae dorso pilosulae rubescentes; flores $\hat{o}$ circ. 8 , breviter pedicellati, pedicellis circ. $0.7 \mathrm{~mm}$. longis glabris, sepala ovata apice acuta circ. $2 \mathrm{~mm}$. longa dorso glabra rubescentia, stamina sepala superantia $3-4 \mathrm{~mm}$. longa, antheris circ. $1 \mathrm{~mm}$. longis, ovarii rudimentum sepalis paullo longiore apice dilatatum; flos $\circ$ terminalis, sepala ovato-oblonga apice acutiuscula glabra circ. $2.5 \mathrm{~mm}$. longa, ovarium stylis crassis brevis paullo longiore glabrum, stigmata obcordata usque ad medium stylum decurentia. Capsula ignota. Fig. 18 a-i, Pl. II, Fig. 2.

China. Western Hupeh: Ichang-gorge, on rocks, alt. 30-300 m., March 24, 1908 (WiLson, no. 3999, type, in HA; fluviatile shrub, 15-30 cm. tall); same locality, Oct. 1887 (A. HenRY, no. 3318, in $\mathrm{HA}$ ).

A very distinct species well characterized by its small oblanceolate leaves with obscure lateral nerves on both surfaces, and 
with the midrib scarcely elevated beneath, and by its somewhat elongated racemes with stalked male flowers with very long gynophores.

This is undoubtedly closely related to $B$. Harlandii HANCE from Hongkong, from which it is readily distinguished by its somewhat smaller obscurely nerved leaves with the midrib scarcely elevated beaneath when dried, its longer gynophores, and by its sepals and bracts marked with numerous rubescent dots on the dorsal side.

var. fukienensis HATUSIMA, var. nov.

A typo recedit foliis paullo majoribus, sepalis bracteisque dorso vix rubescentibus, stylis complanatis longioribus. Fig. $18 \mathrm{j}$-k.

China. Fukien: without precise locality, DunN's Exped., April to June, 1905 (Herb. Bot. Gard. Hongkong, no. 3515, type, in HA).

In its floral structure this variety is most closely related to the type, but in the foliage it is not alike. This variety somewhat resembles $B$. stenophylla HANCE, the type of which was collected at southern Fukien. That species, however, has much narrower and smaller leaves with prominent venation, and sessile male flowers with much shorter gynophores.

In its foliage this bears also some resemblance to B. Bodinieri LÉvL. which has prominently nerved leaves and sessile male flowers.

Of this variety which needs further investigation I have seen only a specimen bearing immature fruits and few male flowers, so without having seen better specimens it is very difficult to decide whether this variety represents a distinct species or a variety.

22. Buxus Bodinieri LÉveILlé in Fedde, Repert. Sp. Nov. 11: 549 (1915); Fl. Kouy-Tchéou, 160 (1914).

Buxus Harlandii HaNCE in Journ. Linn. Soc. 13: 123 (1873), pro parte.

Buxus Harlandii var. platyphylla ScHNEIDER, Illus. Handb. Laubholzk. 2: 139, fig. $90 \mathrm{f}-\mathrm{g}$ (1907), syn. nov.

? Buxus microphylla sensu HaNdel-MazzetTi, Symb. Sinic. 7: 237 (1931), non SiEBOLd et ZUCCARINI.

Buxus microphylla var. platyphylla HANDEL-MAzzeTti, 1.c. excl. syn. et pl. ex Yunnan, syn. nov. 
Buxus microphylla var. aemulans REHDER et WILSON in SARGENT, Pl. Wils. 2: 119 (1914), pro parte (excl. HENRY, no. 7808 et VeITCH Exped., no. 433).-ReHDER in Journ. Arn. Arb. 14: 236 et 426, pro parte.

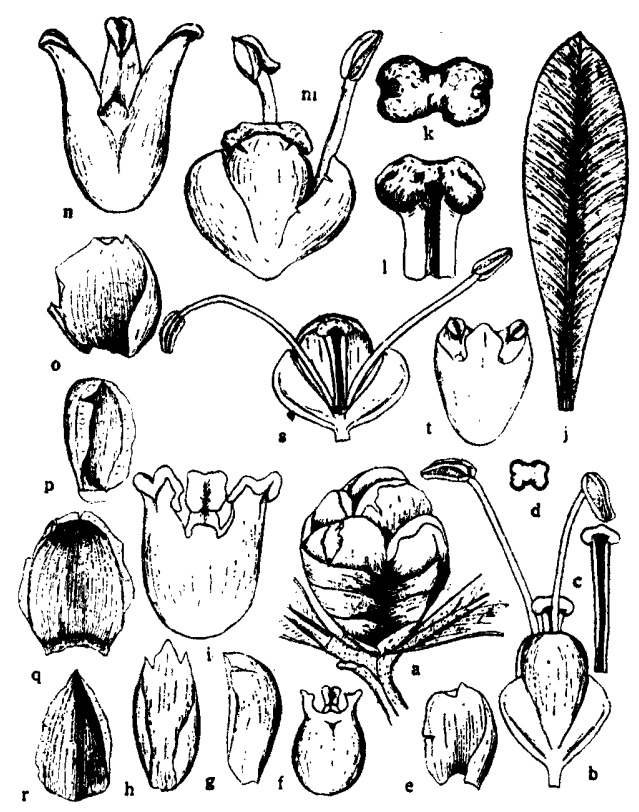

Fig. 19. Buxus Bodinieri LÉvL. (a-i, Herb. Lingnan, no. 13247; j-h, Rock, no. 7932 from Yunnan; s-t, Bodinier, no. 2079; type ex LÉvL). a, branchlet with undeveloped inflorescence $\times 5 ; b$, male flower $\times 5$; $c$, rudimental ovary $\times 5$; $d$, same, seen from above $\times 5$; e, bract, seen from within $\times 5 ; \mathrm{f}$, capsule (Chinc, no. $6319 ; \mathrm{g}, \mathrm{h}$, outer and inner sepals of female flowers, seen from side and within $\times 5$; $i$, ovary $\times 5 ; j$, leaf $\times 1 ; k, 1$, rudimental ovary, seen from above and side; $m$, male flower $\times 5 ; n$, ovary $\times 5 ; 0, p$, internal and external sepals of female flower, seen from within and side $\times 5 ; r$, bracts, seen from different sides $\times 5$; $s$, male flower, inner sepal and two stamens taken off $\times 5 ; t$, premature ovary $\times 5$.

Frutex rami albo-cinerascentes, ramuli hornotini tetragon puberuli vel glabrescentes. Folia tenuiter coriacea, breviter petiolata, oblanceolata vel oblongo-oblanceolata vel anguste obovata 
plerumque supra medium latissima $2-4 \mathrm{~cm}$. (plerumque $2.5-3 \mathrm{~cm}$.) longa, apice obtuse acuta vel mucronulata vel breviter emarginata, basi acuta vel anguste cuneata, margine deorsum leviter anguste revoluta, supra nitidula viridia subtus pallidiora opaca, nervis lateralibus numerosis mediis angulo $45^{\circ}-50^{\circ}$ a costa egressis utrinque in sicco distinctis, costa media utrinque valde elevata subtus cystolithis albidis linearibus vix notata supra in parte inferiore plerumque puberula; petioli margine parce pilosuli circ. $1-2 \mathrm{~mm}$. longi. Inflorescentiae axillares dense glomeratae globosae, rhachi brevissima circ. $2.5 \mathrm{~mm}$. longa, bractearum paria saepe 6 , bracteae ovatae dorso glabrae vel pilosulae luteo-viridescentes; flores $\$$ breviter pedicellati vel subsessiles, sepala circ. $2.5 \mathrm{~mm}$. longa ovato-rotundata convexa dorso glabra vel sparse pilosula, margine anguste scariosa, stamina sepalis 2-4 plo longiora 4-6 $\mathrm{mm}$. longa, antheris circ $1.5 \mathrm{~mm}$. longis, ovarii rudimentum sepala fere aequans vel leviter longiore circ. $2.5 \mathrm{~mm}$. longum apice valde' dilatatum; flos o terminalis, ovarium stylis complantis apicem versus vix angustatis leviter longiore, stigmata obcordata. Capsula ovoidea circ. $7 \mathrm{~mm}$. longa, stylis persistentibus circ. $1.5-2 \mathrm{~mm}$. longis apicem versus vix recurvis. Fig. 19, Pl. IV, Fig. 1.

China. Kwei chou: environs de Kouy-yang, mont du callége, Feb. 26, 1898 (E. BodinIER, no. 2079; arbuste plus ou moins grand; holotype of $B$. Bodinieri ; merotype in HA); highest ridge of Vanchingshan, Yinkiang, alt. 840 m.', Jan. 1, 1931 (TsiaNG, no. 7981; shrub $2 \mathrm{~m}$. ht. ; in HA et HSa); Liang-feng-yah, Tsunyi Hsien, alt. 1100 m., Aug. 12, 1931 (N. Steward, C. Y. Chiao, and H. C. Cheo, no. 284 ; shrub, $80 \mathrm{~cm}$. high., adventitious roots found on stem; Chinese name "Huang-Yang", in HA). Kwangsi: Chin-tung, 80 $\mathrm{Li}$ s. of Nee-bai, border of Kweichow, alt. $800 \mathrm{~m}$., June 30, 1928 (R. C. CHING, no. 6319 ; shrub, $60 \mathrm{~cm}$. high., in rock stream bottom; in HA); Linyen, Aug. 12, 1928 (R. C. CHING, no. 6841, in HSa). Kwangtung: without precise locality (Herb. Lingn., no. 13247, in HSa). Western Hupeh: without precise locality (A. HENRY, no. $3293 \mathrm{~A}$; paratype of $B$. microphylla var. aemulans ex REHDER et WILson; in HA); Changyang Hsien, alt. 1800 m., April 1900 (VerTch Exped, no. 433; shrub, $1 \mathrm{~m}$. high., in HA). Szech'uan: without precise locality, Nov. 28, 1930 (W. FANG, no. 9487, in HSa). Yunnan: Sheweli River drainage basin and environs of Tengyueh, Feb. 1923 (J. F. Rock, no. 7932; small tree 2-3 m., cultivated in 
Shui-yui-ssu (temple court yard; in HA). Honan: Layng, April 28, 1923 (Hero, nos. 3493, 3031; Chinese name "Huang Jang"; in HA). Chekiang: 'Mt. Tienmu-shan, Hsitienmu, Oct. 4, 1934 (SHEN, no. 290; shrub $1 \mathrm{~m}$. ht.; in HSa). Fukien: Diongloh Hsien, April 5, 1923 (H. H. CHUNG, no. 1235, in HA); without precise locality (H. H. ChUnG, no. 6964, in HA; shrub 1.3 m. ht.); Kuling near Fuchau, April 8, 1937 (H. MIGO; planted; in HSa).

REHDER and WILSON confused this box with B. microphylla subsp. sinica var. aemulans HATUSIMA which differs in the lanceolate to ovate leaves with obscure nerves and the less elevated midrib on the beneath, and in the shorter stamens and rudimental ovary of the male flowers. In the narrower leaves this bears some resemblance to the Japanese $B$. microphylla, from which it is easily distinguished by its somewhat larger usually narrowly oblong-oblanceolate leaves with distinctly elevated midrib on the beneath, its longer stamens of the male flower, and its more or less puberulous branchlets, etc.

B. Harlandii var. platyphylla SCHNEIDER of which I have seen only a drawing of the type specimen with the note "This is the only specimen assumed to SCHNEIDER's description" and a detached leaf which agrees well with the author's description. So far as I can see from the drawing and a detached leaf of the type specimen, SCHNEIDER's variety does not seem to differ from the species cited above. I do not hesitate, therefore, to reduce this variety to a synonym of $B$. Bodinieri LÉVL.

HANDEL-MAZzETTI united this variety (Symb. Sinic. 7: 237, 1931) with $B$. microphylla subsp. sinca HATUSIMA which has much thicker coriaceous leaves usually broadest below the middle part. It is very doubtful if the true subsp. sinica occurs as far south as Hongkong where the ScHNEIDER's type was collected. B. microphylla sensu HANDEL-MAZZETTI from Fukien and Kiangsu may possibly belong here.

23. Buxus Harlandii HANCE in Journ. Linn. Soc. 13: 123 (1873), pro major. part.-SchNeIDER, Illus. Handb, Laubholzk. 2: 139, fig. $80 \mathrm{w}, 90 \mathrm{~d}$-e (1907).-REHDER et Wilson in SARGENT, Pl. Wils. 2: 166 (1914), pro parte, excl. pl. ex Hupeh et Fukien.Elwes \& Henry, Trees Gr. Brit. \& Irel. 7: 1722 (1913).-REHDER, Man. Cult. Trees \& Shrubs 533 (1927). 
Buxus sempervirens sensu BENTHAM, Fl. Hongk. 315 (1861)HeMSLEY in Journ. Linn. Soc. 26 : 418 (1894), pro parte.-DUNN \& TUCHTER in Kew Bull. Misc. Inform. add. ser. 10: 233 (1912) (Fl. Kwangt. and Hongk.)-non LinnaEus.

Frutex humilis, cortice albo-cinerascente longitudinaliter irregulariter fisso, rami tetragoni sparse suberosi glabri, ramuli hornotini tetragoni sparse pilosuli vel ferè glabri, graciles circ. $1 \mathrm{~mm}$. diametro. Folia brevissime petiolata, coriacea vel tenuiter coriacea, anguste obovata vel oblongo-obovata vel oblanceolata apice emarginata basi anguste cuneata, margine leviter deorsum anguste revoluta $2-3 \mathrm{~cm}$. (plerumque 2-2.5 $\mathrm{cm}$.) longa $3-10 \mathrm{~mm}$. (plerumque 5-6 mm.) lata, supra nitidula glabra subtus opaca, nervis lateralibus utrinque manifeste distinctis, costa media utrinque valde elevata supra in parte inferiore plerumque puberula; petioli circ. $1-2 \mathrm{~mm}$. longi intus plus minusve pilosuli. Racemi axillares terminalesque dense glomerati globosi, rhachi brevia circ. $4 \mathrm{~mm}$. longa fere glabra, bractearum paria 6-8, bracteae ovato-ellipticae canvexae apice obtuse acutae lutescentes dorso glabrae margine ciliolatae late

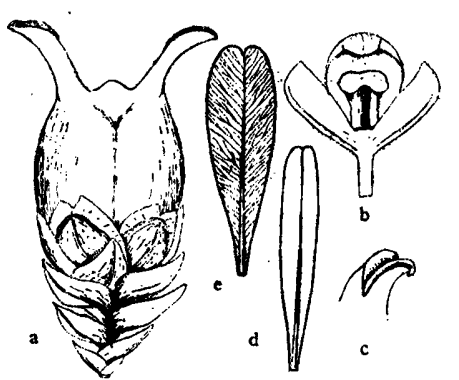

Fig. 20. Buxus Harlandii $\mathrm{H}_{\triangle \mathrm{NCE}}$ (a-d, Chun, no. 5040; b, e, HaN'CE, no. 322, type). a, inflorescence with capsulex 5. b, male flower, inner sepal and stamens taken off $\times 5$. c, stigma magnified. $d$, leaves of different shapes $\times 1$. scariosae circ. $2.5-3.5 \mathrm{~mm}$. longae; flores of distincte pedicellati, pedicellis circ. $1 \mathrm{~mm}$. longis, sepala orbicularia convexa dorso glabra circ. $2.5 \mathrm{~mm}$. longa, stamina sepalis 2-3 plo longiora, ovarii rudimentum sepalis paullo breviore longitrorsum 4-sulcatum apice truncato-capitatum dilatatum; flos $q$ terminalis, ovarium stylis paullo breviore glabrum, stigmata anguste obcordata usque ad medium stylum decurrentia. Capsula nitidula vel opaca glabra cum stylis persistentibus circ. $3 \mathrm{~mm}$. longis apice recurvatis circ. $8 \mathrm{~mm}$. longa; semina trigono-ellipsoidea circ. $4 \mathrm{~mm}$. longa nitida nigerrima. Fig. 20, Pl. V, Fig. 2.

China. Hongkong (HANCE, no. 322, type; paratype et photo. in HA) ; same locality, June 22, 1927 (W. Y. CHUN. no. 5040, in HA; fruiting specimen with narrower leaves than the typical form).

This species is well characterized by its oblanceolate leaves 
with very delicate closely placed branching veins with which the leaves are very marked, and by its pedicelled male flowers.

The nearest relatives to this species are $B$. cephalanthera, $B$. stenophylla and $B$. Bodinieri. It differs from the first in the longer leaves with different venation, in the longer bracts, in the pedicelled male flowers, and in the much larger glabrous capsules with longer horns, etc.; from the sêcond it differs in the larger leaves with different venation, in the pedicelled male flowers, and in the longer bracts; B. Bodinieri has glabrous branchlets with differently nerved leaves and sessile male flowers with longer gynophores.

So far as I can see from the photograph and a part of branchlets of the type of this species, the type specimen, HANCE no. 322, includes two different plants, one of which is true B. Harlandii, and the other represents $B$. Bodinieri. In the original description HANCE describes flowers as follows: "floribus utrinque sexus sessilibus." This must be a mistake. Judging from the specimens before me distribution of this box seems to be restricted near Hongkong.

24. Buxus cephalanthera LÉveILLÉ et VANIOT in FEDDE, Repert. Sp. Nov. 3: 20 (1906).

Buxus sempervirens var. microphylla sensu LÉveILLÉ, Fl. KouyTchéou, 160 (1914).-non Siebold et ZuccarinI.

Buxus Harlandii var.linearis HANDEL-MazzetTI, Symb. Sinic. 7: 237 (1931), syn. nov.

Buxus Harlandii var. cephalanthera REHDER in Journ. Arb. 14: 237 (1933).

Frutex vix ultra $50 \mathrm{~cm}$. altus, dense ramosus, cortice albocinerascente suberoso irregulariter fisso, rami obscure angulares fulvo-virides glabri, ramuli graciles tetragoni plus minusve pilosuli circ. $0.5-1 \mathrm{~mm}$. diametro, internodiis plerumque $5 \mathrm{~mm}$. longis. Folia brevissime petiolata, tenuiter coriacea, anguste obovata vel oblanceolata vel spathulata rarius obovata vel spathulațo-linearia, 0.5$2.2 \mathrm{~cm}$. longa $2-4 \mathrm{~mm}$. lata, apice mucronulata rarius breviter emarginata, basi anguste cuneata, margine anguste revoluta, supra nitidula glabra subtus opaca pallidiora, in sicco nervis lateralibus utrinque distinctis, costa media utrinque valde elevata supra in parte inferiore plerumque puberula; petioli circ. $1 \mathrm{~mm}$. longi ad marginem pilosuli. Inflorescentiae plerumque terminales dense 
glomerate globosae, rhachi plus minusve elongata $3-5 \mathrm{~mm}$. longa dense pilosula, bractearum paria $6-8$, dense pilosula, bracteae ovatotriangulares apice acutae convexae circ. $1.5 \mathrm{~mm}$. longae dorso basim versus pilosulae margine haud scariosae; flores $\hat{o}$ sessiles, sepala ovalia convexa scariosa circ. $1.3 \mathrm{~mm}$. longa dorso glabra, stamina ignota, ovarii rudimentum circ. $2 / 3$ sepala aequans circ. $0.8 \mathrm{~mm}$. longum apice dilatatum truncato-capitatum; flos $\&$ terminalis, sepala ovato-elliptica circ. $1.5 \mathrm{~mm}$. longa glabra, fructibus junioribus ovoideus usque ad $6 \mathrm{~mm}$. longus dense hirtello-velutinus opacus demum glabrescentes laeviusculus, stylis persistentibus lanceolatis $1.5-7 \mathrm{~mm}$. longis, stigmata obcordata usque ad medium stylum decurrentia; semina trigono-ellipsoidea ad 3 $\mathrm{mm}$. longa nitida nigerrima. Fig. 21, Pl. V, Fig. 1.

China. Kweichou: Pin-fa, rochers dans les ruisseaux ou leurs bords, Aug. 25, 1904 (J. Cavalerie, no. 1797; tout petit buis, 1 pied de h.; holotype of $B$. cephalanthera; fragment and photo. in HA); ad flumen infra oppidum Sandjido ad rupes saepe submersas, alt. $370 \mathrm{~m}$., July 17, 1917 (HANDEL-MazZETTI, no. 10809;

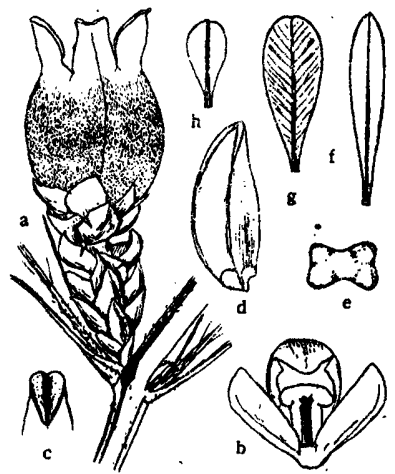

Fig. 21. Buxus cephalanthera LÉvi. (a-f, Handez-Maz., no. 10809). $a$, branchlet with inflorescence bearing capsule $\times 5$. $b$, male flower, inner sepal and stamens taken off $\times 5$. c, stigma magnified. $d$, seed $\times 5$. e, rudimental ovary, seen from above $\times 6$. $\mathrm{f}-\mathrm{h}$, leaves of different shapes $\times 1$ (g, Tsiang, no. 8403 ; $h$, Cavalerie, no. 1797). holotype of $B$. Harlandii var. linearis, in HA); Lungli, Mao-sha, under the bridge, in open, July 4, 1930, (TSIANG, no. 8403; dwarf shrub $60 \mathrm{~cm}$. to $3 \mathrm{~cm}$; ; leaves lustrous green above, light green beneath, fruit brownish-gray ; in HA). Kwangtung: Tai-young-shan, alt. $700 \mathrm{~m}$., July 20, 1921 (F. A. MCCluRE, Herb. Lingn., no. 7215; face of rock; shrub $20 \mathrm{~cm}$. ht., in $\mathrm{HA}$ et $\mathrm{HSa}$ ).

MCCLURE's collection cited above, a very interesting specimen, which needs further investigation, is not typical; leaves oblanceolate to narrowly oblong, mucronate at the apex, about $2 \mathrm{~cm}$. long, thinnly coriaceous. Fruits and male flowers are identical with those of the typical form. Without having seen the flowering specimens I am not sure whether this collection belongs to here or represents a different species. 
- This species is undoubtedly most closely related to $B$. Harlandii, from which it is readily distinguished by its somewhat smaller leaves with less elevated midribs and more acute angle of the less elevated lateral nerves, its sessile male flowers with shorter bracts, and by its smaller capsules with shorter horns which are hirsutevelutinous when young. The type specimen of $B$. Harlandii var. linearis HANDEL-MAZZETTI, of which I have seen a duplicate type is nothing but a narrow-leaved form of this species.

25. Buxus stenophylla HANCE in Journ. Bot. 6: 331 (1868); in Journ. Linn. Soc. 13: 124 (1873).-Mueller-ARG. in DE CANDOLle, Prodr: 16 : 20 (1869).-SCHNEIDER, Illus. Handb. Laubholzk. 2: 139, fig. $90 \mathrm{v}$ (1907). - REHDER et WiLSON in SAREgnt, Pl. Wils. 2: 169 (1914). -Elwes \& Henry, Trees Gr. Brit. \& Irel. 7: 1722, in adnot (1913).

Buxus sempervirens sensu HeMSLEY in Journ. Linn. Soc. 26: 418 (1894), pro parte, quoad syn.-non LinNaEus.

Suffrutescens, rami erecti nigrescento-pubescentes vel fere glabri conferti, cortice albo-cinerascente suberoso, ramuli graciles dense nigrescento-pubescentes circ. 0.7-1 mm. diametro. Folia brevissime petiolata vel lineari-spathulata $1-2 \mathrm{~cm}$. longa $0.2-0.7 \mathrm{~cm}$. lata, apice breviter emarginata vel mucronulata, basi sensim angusta vel anguste cuneata, deorsum leviter revoluta, supra nitidula subtus pallidiora, nervis lateralibus in sicco utrinque

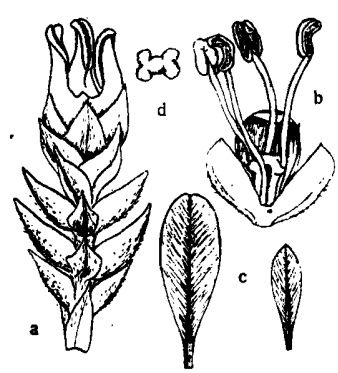

Fig. 22. Buxus stenophylla Hanc: (Levine, no. 403), a, inflorescence, male flowers taken off $\times 4$. b, male flower, inner sepal taken off $\times 5$. c, leaves of different shapes $\times 1$. $d$, rudimental ovary, seen from above $\times 5$. distinctis, costa media utrinque valde elevata supra in parte inferiore plerumque pilosuli. Inflorescentiae axillares et terminales dense glomeratae globosae, rhachi brevissima dense pilosula circ. $3 \mathrm{~mm}$. longa, bractearum paria $6-8$, bracteae ovato-triangulares apice acutiusculae convexae lutescentes circ. $2 \mathrm{~mm}$. longae dorso pilosulae; florés $\hat{s}$ sessiles, sepala ovatoelliptica circ. $2 \mathrm{~mm}$. longa dorso convexa scariosa dorso in parte inferiore pilosula, stamina sepalis circ. duplo longiora, circ. 4 $\mathrm{mm}$. longa, ovarii rudimentum $2 / 3$ sepala aequans circ. $1 \mathrm{~mm}$. longum apice valde dilatatum; flos $q$ terminalis, sepala ovato-triangularia scariosa dorso glabra circ. $2.5 \mathrm{~mm}$. longa, ovarium stylis apice leviter recurvatis 
paullo longiore, stigmata anguste obcordata usque ad $2 / 3$ stylum decurrentia. Capsula ignota. Fig. 22.

China. Kwangtung: Canton and vicinity, Feb. 26, 1917 (Levine, 'no. 403, sub B. Harlandii; in HA). Fukien: Amony, in collibus theiferis Anki, versus fines occidentalis, 1861 (C. DE GRIJs, Herb. HANCE, no. 6683 ; type of HANCE's; fragment in HA).

This apparently distinct species is most closely related to $B$. Harlandii, from which it differs in the smaller leaves with different venation, in the densely pubescent branchlets, and in the sessile male flowers with much shorter bracts. LEviNE's no. 403 from Kwangtung cited above, of which I have given a description above agrees very well with HANCE's description and the type specimen, of which I have seen a part of branchlets.

\section{Group.}

Inflorescentiae spicatae dense glomeratae, rhachi brevissima; folia ovata vel ovato-lancèolata vel elliptica rarius oblanceolata saepissime infra medium latissima apice saepe emarginata vel.obtusa, costa media in sicco subtus leviter obtuso-elevata plerumque cystolithis albidis linearibus longitudinaliter dense notata; ovarium stylis crassis apicem versus sensim dilatatis longiore, stigmata obcordata usque ad medium stylum decurrentia. Capsula glabra lucida. Species 1 Chinae et Japoniae incola.

This group is most closely related to the group 5, but differs from it in the sessile male flowers with longer rudimental ovaries.

26. Buxus microphylla Siebold et ZucCarini in Abh. Akad. Münch. 4: 142, pt. 2 (1845) (Fl. Jap. Fam. Nat. 1:34).-BAIlLoN, Monogr. Buxac. 64 (1859).-KoEHNE, Deutsch. Dendrolog. 356, fig. 51,. j-o (1893).-SCHNEIDER, Illus. Handb. Laubholzk. 2: 140, $90 \mathrm{~m}-\mathrm{n}$ (1908).-Makino in Bot. Mag. Tokyo 27: 113 (1913).-Elwes \& HenRY, Trees Gr. Brit. \& Irel. 7: 1722 (1913).-ReHDER et WILSON in SARgent, Pl. Wils. 2: 165 (1914).

Buxus sempervirens (sphalmate virens) sensu THUNBERG, Fl. Jap. 77 (1784), pro major. part.-non LINNAEUS.

Buxus sempervirens \% microphylla Blume ex Miquel in Ann. Mus. Bot. Lugd. Bat. 3: 128 (1867) pro syn. ; Prol. Fl. Jap. 292 (1867) 
- Hayata in Journ. Coll. Sci. Tokyo, 20: art 3, 83, t. 6 D (1904). -Matumura, Ind. Fl. Jap. 2: 311 (1912).

Buxus japonica a microphylla MUELLER-ARg. apud MiQUEL in Ann. Mus. Bot. Lugd. Bat. 3: 128 (1867); Prol. Fl. Jap. 292 (1867). -Mueller-ARg. in De CANDolle, Prodr. 16: 20 (1869).-FranChET et Savatier, Enum. Pl. Jap. 1: 428 (1875).-OKubo, Cat. Pl. Bot. Gard. Imp. Univ. Tokyo 190 (1887).-DiPPEL, Handb. Laubholzk. 3: 83 (1893).

Buxus sempervirens " angustifolia sensu Siebold, Syn. Pl. Oecon. Jap. 30 (1830).-- non LiNNAEUS.

Frutex glaberrimus ramosissimus, cortice cinerascente suberoso longitudinaliter fisso, ramuli tetragoni glaberrimi graciles circ. $0.5-0.8 \mathrm{~mm}$. crassi, internodiis circ. $0.5-1.5 \mathrm{~cm}$. longis. Folia breviter petiolata tenuiter coriacea vel coriacea, anguste obovata vel oblanceolata vel spathulata rarius obovata plerumque $1.5-2 \mathrm{~cm}$. longa $5 \mathrm{~mm}$. lata, apice brevissime emarginata vel obtusa rarius acutiuscula, basi sensim angustata vel anguste cuneata, margine deorsum leviter revoluta, supra viridia nitidula subtus pallidiora opaca, costa media supra valde elevata subtus haud elevata vel leviter obtusoelevata ${ }^{\cdot}$ cystolithis albidis linearibus longitudinaliter notata, nervis lateralibus in sicco supra distinctis subtus obsoletis; petioli circ. 1-1.5 mm. longi glabri. Inflorescentiae axillares vel terminales dense

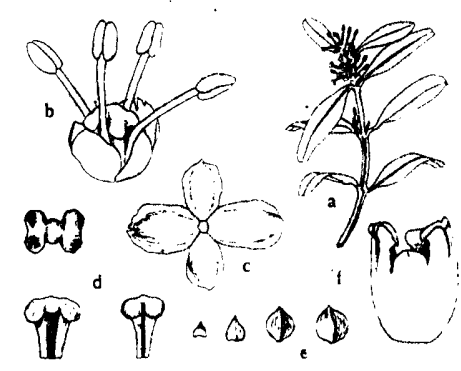

Fig. 23. Buxus microphylla Sier. et Zucc. a, flowering branchlet $x$ $1 / 2$. b, male flower $\times 2.5$. c, sepals of same, seen from with out $\times 2.5$. d, rudimental ovaries seen from different sides $\times 4$. $e$, bracts of different shapes, seen from without $\times 2.5$. $\mathrm{f}$, ovary $\times 4$. glomeratae globosae, rhachi brevissima glabra, bractearum paria 4 , bracteae late ovatae vel orbiculares convexae circ. 1-2 mm. longae dorso glabrae apice acutiusculae margine anguste scariosae; flores o sessiles paria 2-3, sepala obovato-elliptica vel orbicularis plerumque $2.5-3 \mathrm{~mm}$. longa convexa glabra margine scariosa, stamina sepala superantia circ. $4-5 \mathrm{~mm}$. longa, antheris ovatis $1.4-2.25 \mathrm{~mm}$. longis, apice apiculatis luteo-flavescentibus, ovarii rudimentum sepala fere aequans circ. 1.5 $\mathrm{mm}$. longum apice dilatum truncatotetragonum 4-sulcatum circ. $1.3 \mathrm{~mm}$. latum $1 \mathrm{~mm}$. longum; flos terminalis, ovarium stylis brevis crassis apicem 
versus plus minusve dilatatis longiore circ. $3 \mathrm{~mm}$. longum $2 \mathrm{~mm}$. latum, stigmata obcordata usque ad medium vel $2 / 3$ stylum decurrentia. Capsula ovoidea cum stylis persistentibus divaricatis circ. $2 \mathrm{~mm}$. longis circ. $1 \mathrm{~cm}$. longa. Semina ignota. Fig. 23, Pl. III, Fig. 1.

Japan. Kyūsyū: Fukuoka, cultivated, March 31, 1938 (HATUSIMA; shrub $30 \mathrm{~cm}$. ht.).-Prov. Hyuga, cultivated, alt. $800 \mathrm{~m}$., Dec. 25, 1938 (Hatusima, no. 10355). Honsyu: Prov. Rikuzen, Oct. 15, 1933 (T. INOKUMA, nos. 3860, 3861; cultivated).

This Box so long cultivated in gardens is without doubt a native of Japan, but it has not yet been collected in a wild state and the Japanese botanists apparently do not know about its habitat. So I am now inclined to believe that it may probably be a garden form originated from cuttings and subsequently propagated by cuttings. Most of the wild forms which were identified with this species by most authors seem to be an extremely narrowleaved form of the following var. japonica REHD. et WILS.

It is an interesting fact that this Box often produces numerous adventitious rootlets vigorously on the stem which are scarcely seen in var. japonica REHD. et WILS.

The inflorescence of this Box is considered by most botanists to be usually terminal, but this is not constant character as shown in the accompanying plate and I do not consider that it has taxonomic value.

HANDEL-MAZzeTtr records (Symb. Sinic. 7: 237, 1931) this Box from Fukien and Kiangsu, but its occurrence there is very doubtful; very likely specimens of $B$. Bodinieri LÉVL. have been erroneously determined as $B$. microphylla SIEB. et Zucc.

var. japonica ReHDER et Wilson in SARgent, Pl. Wils. 2: 168 (1914).

Buxus sempervirens (sphalmate virens) sensu THUNBERG, Fl. Jap. 77 (1884), pro minor. part.-HEMSLEY in Journ. Linn. Soc. 26 : 418 (1894), pro parte.-MatumuRA, Ind. Fl. Pl. Jap. 2: 310 (1912). -non LinNaEus.

Buxus sempervirens $\beta$ suffruticosa sensu Siebold, Syn. Pl. Oecon. Jap. 30 (1830).-non LiNNAEUS. 
Buxus japonica Mueller-Arg. apud Mrquel in Ann. Mus. Bot. Lugd. Bat. 3: 128 (1867); Prol. Fl. Jap. 292 (1867).-MuELLER-ARG. in De Candolle, Prod. 16: 20 (1867).-Franchet et Savatier, Enum. Pl. Jap. 1: 428 (1875).-OKUBo, Cat. Pl. Bot. Gard. Imp. Univ. Tokyo, 190 (1887).-PAX in ENGLeR \& Prantl, Nat. Pf.fam. 3 (5) : 130 (1890).-DipPEL, Handb. Laubholzk. 2: 139 (1893), pro parte.-SCHNEIDER, Illus. Handb. Laubholzk. 2: 139, fig. v-x (1907).-SiraSaWA, Icon. Forest. Trees Jap. 2: 92, fig. 38 (1909).MAKINo in Bot. Mag. Tokyo 27: 112 (1913).-Elwes \& Henry, Trees Gr. Brit. \& Irel. 7: 1721 (1913).--Makino et Nemoto, Fl. Jap. ed. 2: 663 (1931).

Buxus sempervirens var. japonica Makino in Bot. Mag. Tokyo 9: 281 (1895); 15: 169 (1901).-Hayata in Journ. Coll. Sci. Tokyo, 20: art. 3, 82, t. 6, fig. C (1904).

Buxus sempervirens var. riparia Makino in Bot. Mag. Tokyo 26: 293 (1912).

Buxus microphylla var. riparia Makino in Bot. Mag. Tokyo 27: 113 (1913).-MAKino et Nemoto, Fl. Jap. ed 1: 630 (1925); ed. 2: 663 (1931).-Masamune in Mem. Facult. Sci. \& Agric. Taihoku Imp. Univ. 11: 273 (1934)

Buxus microphylla var. arborescens NAKAI, Tennen-KinenbutuTyôsahôku, 7: 71 (1927), cum diagn. Jap. brev.

Buxus microphylla var. rotundifolia NAKAI, 1.c. cum diagn. Jap. brev.

Buxus riparia Makino in Asahina's Journ. Jap. Bot. 7: 14 (1931).

Buxus microphylla var. suffruticosa (SIEBOLD) MAKINo in Zitssaiengei, 25: 529 (1935), syn. nov.

Buxus microphylla var. suffruticosa form. riparia MAKINo, 1.c.

Buxus microphylla var. suffuticosa form. minutissima MAKino, 1.c. cum fig., syn. nov.

Buxus microphylla var. suffruticosa form. tenuis MAKINO, l.c. cum fig., syn. nov.

Frutex vel arbor parva glaberrima, cortice cinerascente, rami albo-cinerascentes longitudinaliter striati decorticates, ramuli hornotini glabri tetragoni luteo-viridescentes circ. $1 \mathrm{~mm}$. crassi, 'internodiis plerumque $1-2 \mathrm{~cm}$. longis. Folia ovata vel ovato-elliptica 
rarius oblongo-oblanceolata vel ovato-rotundata, coriacea, plerumque $1-1.5 \mathrm{~cm}$. longa $0.6-1 \mathrm{~cm}$. lata (maxima $2 \mathrm{~cm}$. longa $1.4 \mathrm{~cm}$. lata), apice breviter emarginata vel obtusa rarius acutiuscula, basi cuneata vel anguste cuneata vel sensim angustata, margine deorsum leviter anguste revoluta, supra nitida viridissima subtus pallidiora opaca, costa media supra valde elevata subtus haud elevata vel leviter, obtuse elevata cystolithis albidis linearibus longitudinaliter densissime notata, nervis lateralibus in sicco supra distinctis subtus obsoletis; petioli circ. 1-1.5 mm. longi glabri. Inflorescentiae axillares dense glomeratae globosae, rhachi glabra brevissima, bractearum paria 5 , bracteae late ovatae acutae convexae margine anguste scariosae dorso glabrae circ. $2 \mathrm{~mm}$. longae; flores $\hat{o}$ sessiles paria plerumque 3 , sepala fere orbicularis convexa membranacea apice obtuse acuta dorso glabra circ. 2-2.5 mm. longa, stamina sepalis circ. duplo longiora 4-4.5 mm. longa, antheris circ. $1.5 \mathrm{~mm}$. longis apice apiculatis, ovarii rudimentum sepala fere aequans longius stipitatum circ. $2 \mathrm{~mm}$. altum apice capitatotruncato-tetragonum circ. 1.7 $\mathrm{mm}$. latum; flos 우 terminalis,

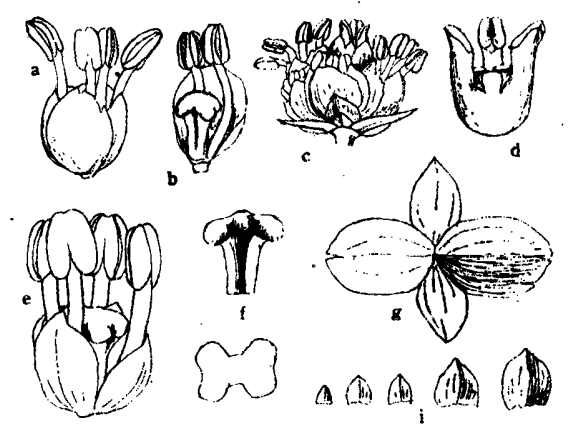

Fig. 24. Buxus microphylla var. japonica REHD. et $W_{\text {LLS. }}$ a, male flower in early stage $\times 3.5$. b, same, showing rudimental ovary $\times 3.5$. c , inflorescence $\times 2$. d, ovary $\times 2.5$. e, male flower in the latter stage $\times 4.5$. $\mathrm{f}$, rudimental ovary, seen from side and above $\times 5$. $g$, sepals of same, seen from without $\times 5$. 1, bracts of different shapes, seen from without $x$ 2.5 . sepala late ovata circ. $2.5 \mathrm{~mm}$. longa glabra scariosa, ovarium stylis brevis crassis apicem versus sensim plus minusve dilatatis paullo longiore, stigmata obcordata usque ad medium stylum decurrentia. Capsula globoso-ovoidea cum stylis persistentibus crassis valde divaricatis circ. $3 \mathrm{~mm}$. longis circ. $1 \mathrm{~cm}$. longa; semina trigono-ovato-ellipsoidea lucida nigerrima circ- $5 \mathrm{~mm}$. longa. Fig. 24, P1. XII.

Japan. Kyūsyū: Island of Yakusima, Prov. Satuma, alt. 1600 m., on granite, July 17, 1933 (T. NAITô); same locality, alt. 1800 m., Aug. 1936 (Matuoka; shrub. $1.5 \mathrm{~m}$. ht., common); Island of Tusima, Prov. Tusima, on limestone, rare, Aug. 2, 1933 (K. NAKA- 
SIMA) : Kônose, Prov. Higo, on limestone, BARA, nos. 3508, 3510); Ôno, same locality, on limestone, rare, Oct. 1, 1916 (Mayebara, no. 3509); Mt. Kosyo-zan, Prov. Tikuzen, on limestone, common (Hatusima ; KusaKa; Dor ; Kirihara; MoriKAWA); Mt. Kyôgadake, Prov. Hizen, alt. 1300 m., on andesite, Sept. 23, 1938 ( $\because$ Toyama). Sikoku: Sakawa, Prov. Tosa, on limestone, common, April 17, 1636 (KuSAKa). Honsyû: Atrimura, Nisimurogun, Prov. Kii, Sept. 28, 1924 (UI, nos. 2432, 2434); Dorokyô, Higasimurogun, Prov. Kii, Sept. 28, 1926 (UI, no. 3436); Mt. Fujiwaradake, Prov. Ohmi, Dec. 14, 1938 (S. Окамото); Mt. Ohdaigahara, Prov. Yamato, alt. 1700 m., Aug. 5, 1936 (Y. KATô); Mt. Zinko-zan, Prov. Mikawa, common, Aug. 20, 1933 (Y. KATô); Mt. Amagi, Prov. Idu, Sept. 1909 (T. MAKINo, in HT); without locality and date (MAYR, in HA); Hyôkawamura, Okutama, Prov. Musasi, Nov. 22, 1936 (KUSAKA); Umiya, Nisiumimura, Nisikubikigun, Prov. Yetigo (M. TAKAHASI).

I am unable to follow MUELLER and others in distinguishing B. microphylla and B. japonica as species. There is apparently no floral characters by which to separate them, even the shape of the leaves upon which much stress is laid is considerably variable.

This Box is widely spread, though it is rather local and peculiar, in Japan from the island of Yakusima off the extreme south of Kyūsyu northward to the northern part of Honsyû (Lat. $38^{\circ} 33^{\prime} \mathrm{N}$ ), and also commonly planted for ornament. On Mt. Kosyo-zan, a limestone mountain, in the province of Tikuzen in Kyūsy $\bar{u}$, a most famous pure wood of this Box exists which must be of great age. This wood is confined to mountain peak at an elevation of $800 \mathrm{~m}$. to $860 \mathrm{~m}$., and extends for about 11 hectares, with a total content of about $260 \mathrm{fm}$. . Most of the Box tree attain 3-5 m. in height, and $10-20 \mathrm{~cm}$. in diameter, the largest tree which was in 1927 measured by the forester, was $12 \mathrm{~m}$. by $53 \mathrm{~cm}$. at the base and $37.5 \mathrm{~cm}$. at $1 \mathrm{~m}$. from the ground. A Box tree measuring about $18 \mathrm{~cm}$. in diameter at $1 \mathrm{~m}$. from the ground, when in 1927 cut down, was estimated to be about 280 years old.

form. rubra (MAKINO) HATUSIMA, comb. nov.

Buxus sempervirens var. japanica f. rubra MAKINo in Sched. in Herb. Sci. Coll. Imp. Univ. Tokyo. 
Buxus japonica f. rubra Makino in Bot. Mag. Tokyo 27: 112 (1913).

Frutex, folia ovata vel oblonga, coriacea $3-12 \mathrm{~mm}$. longa 2-7.5 $\mathrm{mm}$. lata, aurantiaca, nervis lateralibus in sicco supra prominentibus subtus obsoletis. Flores paullo minores.

Japan. Cultivated.

This is a garden form with orange coloured leaves. I have not seen any specimens.

DiPPEL describes (Laubholzkunde 3: 83, 1893) under the name B. japonica a variegated form, var. variegata, the leaves of which are margined with white or yellow; I am not quite sure, whether this variety belongs either to the Japanese var. japonica or to Chinese species, since his $B$. japonica includes besides those enumerated above the Chinese species which may probably represent $B$. microphylla subsp. sinica, and he says nothing about the origin of his plants.

form. major (MAKINo) Hatusima, comb. nov.

Buxus Kitashimai YanAgidA in Journ. Sci. Forest. Jap. 15: 931, fig. 391 (1933) cum diagn. Jap. brev.

Buxus microphylla var. suffruticosa f. major MAKINO in Zitssaiengei, 25: 529 (1939).

A var. japonico recedit ramis ramulisque crassioribus minus ramosis, foliis majoribus ad $3.5 \mathrm{~cm}$. longis $1.7 \mathrm{~cm}$. latis, floribus paulo majoribus.

Arbor parva glaberrima, maxima usque ad $6 \mathrm{~m}$. alta $30 \mathrm{~cm}$. diametro, cortice luteo-cinerascente longitudinaliter irregulariter fisso, rami albo-cinerascentes decorticantes valde rugosi, ramuli hornotini luteo-viridescentes tetragoni $1-1.5 \mathrm{~mm}$. crassi, internodiis $1-2 \mathrm{~cm}$. longis. Folia crasse coriacea, ovato-oblonga vel ovato-elliptica rarius anguste elliptica ad $3.5 \mathrm{~cm}$. longa $1.7 \mathrm{~cm}$. lata, apice plerumque breviter emarginata, basi acuta vel breviter acuminata, margine valde anguste revoluta, in sicco supra nitida glabra subtus pallidiora, costa media supra valde elevata glabra subtus leviter elevata cystolithis albidis linearibus longitudinaliter dense notata, nervis lateralibus in sicco supra distinctis subtus obsoletis. Inflorescentiae axillares dense glomeratae, rhachi brevissima glabra $2-3 \mathrm{~mm}$. longa, bractearum paria $4-5$, bracteae convexae apice plerumque bifidae 


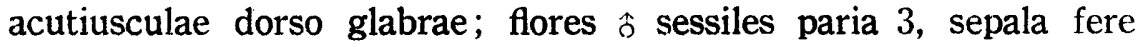
orbicularis apice obtuse acuta membranacea flavescentia dorso glabra circ. $2-2.5 \mathrm{~mm}$. lata, stamina sepala superantia circ. $5.5 \mathrm{~mm}$. longa, ovarii rudimentum sepala fere aequans vel paulo longiore 2-3 $\mathrm{mm}$. longum apice valde dilatatum truncato-tetragono-capitatum circ. $1.8 \mathrm{~mm}$. latum rugosissimum; flos 9 terminalis, sepala ovata membranacea, apice obtuse acuta saepe bifida $2.5 \mathrm{~mm}$. longa, ovarium stylis divaricatis apicem verus sensim dilatatis et leviter recurvatis paullo longiore, stigmata elliptico-obcordata. Capsula ignota. Pl. III, Fig. 2.

Japan. Honsyu: Prov. Izu: Hatijô, May 6, 1917 (WiLson, no. 8377; bush 3-10 ft., planted, in HA); Isl. Osima, March 16, 1907 (K. SAKURAI; in HA), Isl. Mikurajima, July 5, 1939 (TAKAHASI).

This form with large leaves up to $3.5 \mathrm{~cm}$. long, is apparently only a luxuriant form owing its origin to the moist subtropical climate of the islands where it grows. According to Dr. Syôtarô HoRI, this peculiar form is said to occur wild on the island of Mikurajima, off the extreme southern end of Izu-Hitito Group where it grows naturally between $400-850 \mathrm{~m}$. and extend about 200 hectares with an estimated total number of about 205,000 trees.

subsp. sinica (REHDER et WILSON) comb. nov.

Buxus sempervirens sensu HeMSLEY in .Journ. Linn. Soc. 26 : 418 (1894), pro parte.-Diels in ENGLER, Bot. Jahrb. 29 : 431 (1900). -E. Gilg in ENGler, Bot. Jahrb. 34: Beibl. 75: 49 (1904).-LoESENER in Bot. Centralb. Beih. 37: 150, pt. 2 (1914).--HAYATA, Mont. Fl. Formos. in Journ. Coll. Sci. Imp. Univ. Tokyo, 25: art. 3, 193 (1908).-PAMPaninI in Nuov. Giorn. Bot. Ital. n. ser. 18: 126 (1911). -non LinNaEus.

Buxus microphylla var. sinica REHDER et WILSON in SARGENT, Pl. Wils. 2: 165 (1914).-Hers in Journ. N. China Branch R. As. Soc. 53: 107 (1922); Liste Ess. Lign. Honan Sept. 5 (1922).-CHUNG in Mem. Sci. Soc. China, 1: 137 (1931) (Cat. Trees \& Shrubs China). -ReHDER in. Journ. Arb. 7: 194 (1926); 8: 154 (1927).—KANEHIRA, Formos. Trees, 476 (1917).-SASAKI, List. Pl. Formos. 267 (1928).-Makino et Nemoto, Fl. Jap. ed. 2, 664 (1931).

Buxus intermedia KanehIRA, Formos. Trees rev. ed. 359, fig. 315 (1936), excl. fig. B et C, cum diagn. Jap. brev., syn. nov. 


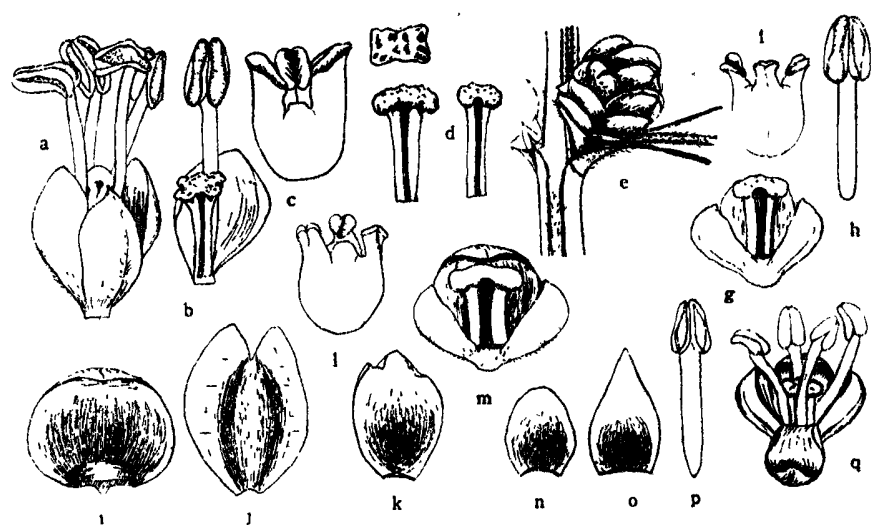

Fig. 25. Buxus microphylla subsp. sinica Hatusima, a-p (a-d, from Formosa; e-h, WiLson, no. 3397, from Hupeh; i-p, WiLson, no. 3398, from Hupeh, a small-leaved form). q, var. aemulans Hatusima (Henry, no. 7807, from Hupeh). a, male flower $\times 4$. b, same, showing rudimental ovary $\times 4$. c, ovary $\times 4$. d, rudimental ovaries, seen from different sides $\times 4$. e, part of flowering branchlet $\times 4$. f, ovary $\times 5, \mathrm{~g}$, male flower, inner sepal and stamens taken off $\times 4$. $h$, stamen $\times 4$. $i$, sepal of male flower, seen from within $\times 5$. $j$, sepal of female flower, seen from without $\times 5$. $k$, bract, seen from within. 1, ovary $\times 3$. m. male flower, showing rudimental ovary $\times 5$. $n, o$, bracts of different shapes, seen from within $\times 5$. p, stamen $\times 4$. $q$, male flower $\times 4$.

Frutex 1-6 m. altus, rami albo-cinerascentes valde striati decorticantes, ramuli tetragoni plus minusve pubescentes. Folia coriacea breviter petiolata, ovato-elliptica vel ovato-oblonga, apice plerumque breviter emarginata vel obtusa, basi cuneata vel acuta, margine valde revoluta, $1.5-3 \mathrm{~cm}$. longa $0.7-1.7 \mathrm{~cm}$. lata, supra in sicco luteoviridia lucida glabra, costa media supra valde elevata in parte inferiore saepe puberula, subtus leviter elevata cystolithis albidis linearibus dense notata, nervis lateralibus in sicco supra distinctis subtus obsoletis; petioli $1-2 \mathrm{~mm}$. longi plus minusve puberuli. Inflorescentiae axillares dense glomeratae globosae, rhachi brevia dense pilosula vix ultra $5 \mathrm{~mm}$. longa plerumque $3 \mathrm{~mm}$. longa, bractearum paria $6-8$, bracteae late ovatae convexae acutae lutescentes dorso plus minusve pilosulae circ. $2.5 \mathrm{~mm}$. longae; flores $\hat{o}$ sessiles, sepala ovato-rotundata convexa $2-2.5 \mathrm{~mm}$. longa dorso glabra margine scariosa, stamina sepalis circ. duplo longiora, antheris circ. $1.5 \mathrm{~mm}$. longis, ovarii rudimentum sepala fere aequans apice valde 
dilatatum tetragonum; flos $i$ terminalis, ovarium stylis apicern versus plus minusve dilatatis paullo longiore, stigmata obcordata usque ad medium stylum decurrentia. Capsula cum stylis persistentibus valde divaricatis circ. $2-3 \mathrm{~mm}$. longis circ. $8-10 \mathrm{~mm}$. longa; semina trigono-ellipsoidea lucida nigerrima circ. $5 \mathrm{~mm}$. longa. Fig. $25 \mathrm{a}-\mathrm{p}$, Pl. VI, VII Fig. 1.

China. Western Hupeh: Changyang Hsien, rocky places, alt. 1000-1600 m., common, May and October 1907 (WiLson, nos. 3397, type, 3396, in HA); Wuchang, April 1933 (S. C. Sun, no. 1149, in HA); same locality, Oct. 8, 1932 (S. C. Sun, no. $45 ; 3$ m. ht. 8.7 cm. in diam., in HA). Szech'uan: Nanchuan Hsien, May 20, 1928 (W. P. FANG, no. 843 ; in thickets, $6 \mathrm{~m}$. ht., in HA). Anhwei: Wang-shan, under woods, alt. $900 \mathrm{~m}$., common, July 13, 1926 (CHING, no. 3000 ; a low dense shrub, $2 \mathrm{~m}$. ht., bark brownish green, smooth ; in HA); same locality, alt. $550 \mathrm{~m}$., along shaded stream, common, July 11, 1925 (CHING, no. 2943; a slender shrub, $3 \mathrm{~m}$. ht.; in HA). Kiangsu: Nanking (E. FABER, no. 902, in HA); same locality, March 28, 1917 (FABER, no. 509, in HA); Sen-tomb, Nanking, April 10, 1931 (CHEN \& TeNG, no. 4103; in HSa); Yun-dai-shan, Haichow, Oct. 21, 1932 (CHANG \& CHENG, no. 899; shrub, $3.5 \mathrm{~m}$. ht., in open place; in HSa); Heo-kia-chen, Tsang-ming, July 22, 1926 (Tso, no. 1520, in HA; small tree $3 \mathrm{~m}$. ht.); Hwashen temple, Nanking, in fence, May 1926 (Tso, no. 1034; in HA; shrub 1.5 m. ht.) ; Huang-tsang-yü, Siao Hsien, May 26, 1919 (Hers, no. 1028; local name; huang yang; in HA); Soochow, Oct. 26, 1929 (S. W. KANG, no. 95 ; in HSa). Shantung: Cape ya-tau, near Taiching-kung, in the valley, 1903 (ZIMMERMANN, no. 529; in HA); Pai-yong-tung, Lao-shan, $120 \mathrm{Li}$ from Tsingtao, alt. $600 \mathrm{~m}$., along rocky slope, July 14, 1930 (CHIAO, no. 2794 ; shrub $4 \mathrm{~m}$. ht.; in $\mathrm{HA}$ ); Ching-shan, Lao-shan, alt. $400 \mathrm{~m}$., along rocky slope, July 12, 1930 (CHIAO, no. 2893).

Japan. Formosa: Prov. Taihoku, Police station, near Tyôsakei, May 27, 1918 (WILson, no. 10205; cultivated ; in HA); round Sôzan, Dation Range, Dec. 13, 1918 (WILSON, no. 11228; planted but said to be local; shrub $3 \mathrm{~m}$. ht., $30 \mathrm{~cm}$. in girth; in HA); Tikusiko (SASAKI, HT, nos. 15111, 15112, 15113); same locality, alt. $90 \mathrm{~m}$., July 26, 1935 (S. SuzuKI); Horisya, Dec. 4, 1912 (Y. KikUTI, HT, no. 15099); Prov. Taitô, Mt. Rontaburu, April 17, 1910 (U. MoRI, HT, no. 15105, in HT); without locality, presumably same locality, 
April 1907 (S. KonisI, HT, no. 15106, in HT); same locality, Tyakankei, Jan. 1908 (U. MoRI, HT, no. 15115, in HT).

This is one of the commonest and most widely dispersed species to be found in central and northern China, and most closely related to $B$. microphylla var. japonica REHDER et WILSON which differs in the glabrous branchlets with usually smaller leaves. It shows the same variation as to the size and form of the leaves as appear in the Japanese var. japonica; the degree of pubescence of the branchlets, petioles, and bracts and sepals is also very variable.

After a careful examination of the abundant material before me I have failed to detect any sufficient floral characters by which to separate the Chinese form from the Japanese in a satisfactory way except that the former has more or less hairly bracts. and sepals. So I am now inclined to think it better to keep the Chinese form as a geographical subspecies of $B$. microphylla on account of its pubescent branchlets and flowers, and its well defined geographical range.

This is also closely related to $B$. rugulosa Hatusima from Yunnan, with which it has been confused by most previous authors, but the Yunnan species has rugose obscurely nerved leaves and shortly stalked male flowers with much shorter rudimental ovaries.

On account of the variation in size and form of the leaves, it is exceedingly difficult to determine what may be regarded as definite varietal lines for subsp. sinica. The above series of collections are reasonably uniform. In addition to these, I have two other groups which with better representation may prove to be a good variety.

The first group cited below is well characterized by its ob- long leaves usually very prominently veined above; the range of this form seems to be restricted to north-western China.

Shensi: Tai-pei-shan, 1910 (Purdom, nos. 1150, 1149, in HA). S.W. Kansu : near Siku, alt. 1500 m. and over, Nov. 17, 1914 (MEYER, no. 2009; amidst stony debris; shrub also small tree; in HA).

The second group differs from the typical form in the slender branchlets with smaller leaves usually not more than $1 \mathrm{~cm}$. long and in the smaller capsules, and might be considered a distinct variety if it did occupy a different geographical area. This group is most closely related to var. insularis Hatusima from Korea and B. rugulosa subsp. prostrata Hatusima from Yunnan, but it 
differs from the first in the distinctly veined upper surface of the thinner leaves with less revoluted margines; B. rugulosa subsp. prostrata HATUSIMA is readily distinguished by its much smaller rugose leaves with strongly revoluted margines, its prostrate habit, and by its different structure of the male flower.

Western Hupeh: Hsing-shan Hsien, woodland, cliff, alt. 13002400 m., common, May 14, 1907 (WILson, no. 3398a, in HA; bush 1-2 m.); same locality, May 5, 1907 (WILSON, no. 3398a, in HA; bush 1-1.5 m.); without locality (VEITCH Exped., no. 433a, in HA); Wan-tsao-shan, in forest under shade of beech and maples, alt. 1500 m., common undershrub, Aug. 18, 1922 (W. Y. CHUN, no. 3944, in $\mathrm{HA}$; shrub 2-3 m. ht., $5 \mathrm{~m}$. tall, $50 \mathrm{~cm}$. diam. were met with).

Anhwei: without precise locality, Dec. 27, 1933 (S. CHEN, no. 2637, in HA).

It is very interest to report that in the province of Satuma in southern Kyūsyū, Japan, this Chinese Box tree has long been intensively planted for timber purpose from 200-300 years ago. At Eimura, a most famous timber-producing village in the same province, according to the report of a forester in 1926, there were total number of about 60,000 trees larger than $15 \mathrm{~cm}$. in diameter, and produced about $400 \mathrm{fm}$. of timber annually.

This Box grows faster than the Japanese Box tree, namely $B$. microphylla var. japonica, and reaches about $13-15 \mathrm{~cm}$. in diameter in 20 years old.

var. aemulans (REHDER et WiLSON) HATUSIMA, comb. nov.

Buxus microphylla var. aemulans REHDER et WILSON in SARGENT, PI. Wils. 2: 169 (1914), pro parte, excl. HENRY, no. 3293a.

Frutex 1-2 m. altus, ramuli compresse tetragoni pauce pilosuli vel fere glabri circ. $1 \mathrm{~mm}$. diametro. Folia ovato-lanceolata vel lanceolata breviter petiolata, coriacea, $2.7-4.3 \mathrm{~cm}$. longa (plerumque circ. $4 \mathrm{~cm}$.) $0.7-1.3 \mathrm{~cm}$ lata (plerumque $1 \mathrm{~cm}$.), apice obtuse acuta, basi cuneata, supra nitidula glabra subtus pallidiora, costa media utrinque elevata, nervis lateralibus in sicco supra distinctis subtus obsoletis; petioli 1-1.5 mm. longi plus minusve pilosi. Inflorescentiae axillares dense glomeratae globosae, rhachi brevia circ. $2 \mathrm{~mm}$. longa pilosa, bractearum paria circ. 6 , bracteae convexae apice obtuse acutae pallide brunnescentes dorso pilosulae circ. $1.5 \mathrm{~mm}$. longae; flores $\hat{o}$ sessiles paria saepe 3 , sepala late ovata vel late elliptica 
circ. $2 \mathrm{~mm}$. longa dorso glabra, stamina sepala paullo superantia circ. $2-2.5 \mathrm{~mm}$. longa, antheris late ellipticis circ. $0.7 \mathrm{~mm}$. longis, ovarii rudimentum sepalis paullo breviore circ. $1.5 \mathrm{~mm}$. longum apice truncato-capitatum dilatatum 4-sulcatum; flos 9 terminalis, ovarium stylis apice leviter recurvatis apicem versus sensim dilatatis paullo longiore, stigmata usque ad medium stylum decurrentia. Fig. $25 \mathrm{q}$, PI. IV, Fig. 2.

China. Western Hupeh: without precise locality (A. HENRY, no. 7807 ; type, in HA); Chang-yang Hsien, woodland, alt. $1800 \mathrm{~m}$., April 1900 (VEITCH Exped. no. 433; in HA); Ta-che-yuan, alt. 2400 m., Sept. 5, 1922 (W. Y. Chun, no. 4235; shrub, 2 m. ht., in HS). Anhwei: Wangshan, Oct. 16, 1933 (W. C. CHENG, no. 4009; in HS). Eastern Szech'uan: Tschen-kéou-tin District (FARGES; in HA).

This variety seems to differ from subsp. sinica in the ovatelanceolate to lanceolate leaves with the more acute apices, and in the shorter stamens. The type specimen of this variety is represented by two quite different specimens of the same number which are mounted on one sheet. One of the specimens which is mounted in the midst of the sheet apparently represents the type of this variety, while the other specimen enclosed in an envelope pasted to the lower left-hand corner of the sheet, and bears the notes "7808, type" on its sheet is, so far as I can see without doubt a narrow-leaved form of $B$. Wallichiana. I suppose that a Himalayan specimen has erroneously been attached on one sheet, as it is very doubtful if the true $B$. Wallichiana occurs in China.

var. insularis (NAKaI) Hatusima, comb. nov.

Buxus japonica sensu Palibin in Acta Hort. Petrop. 18: 188 (1900)-non MUELLER-ARG.

Buxus microphylla sensu NAKAI, Fl. Quelpaert Isl. 60 (1915), non SiEBold et ZuCCARINI.

Buxus microphylla var. sinica REHDER et WILSON in SARGENT, Pl. Wils. 2: 165 (1914), pro parte.-MorI, Enum. Corea. Pl. 235 (1922).

Buxus microphylla var. riparia sensu TROLLOPE in Trans. Korea. Branch Roy. Asia. Soc. 11: 42 (1920).-non Makino.

Buxus microphylla var. insularis NAkAI in Bot. Mag. Tokyo 36 : 63 (1922). 
Buxus microphylla var. koreana NAKAI apud WILSON in Journ. Arn. Arb. 1: 35 (1920), nom. nud.-KEHDER in Journ. Arn. Arb. 7: 240 (1926), syn. nov.

Frutex erectus plerumque $25-60 \mathrm{~cm}$. altus dense ramosus, rami cinerascentes decorticantes rugosi, ramuli hornotini tetragoni plus minusve puberuli circ. $1 \mathrm{~mm}$. diametro, internodiis $0.5-1.5 \mathrm{~cm}$. longis. Folia breviter petiolata, crasse coriacea, ovato-elliptica vel ovata vel elliptica rarius oblonga vel obovata, $1-1.8 \mathrm{~cm}$. longa 0.5 $1 \mathrm{~cm}$. lata, apice saepissime breviter emarginata vel obtusa rarius acuta, basi acuta vel cuneata, margine valde anguste revoluta, supra nitidula viridissima subtus pallidiora, costa media supra leviter elevata in parte inferiore plerumque plus minusve puberula subtus haud elevata vel leviter obtuse elevata cystolithis albidis linearibus longitudinaliter dense notata; petioli 1-1.5 mm. longi plus minusve puberuli. Inflorescentiae axillares dense glomeratae globosae, rhachi dense puberula brevia circ. $2 \mathrm{~mm}$. longa, bractearum paria cic. 5-6, bracteae ovatae acutae convexae pallide rubescentes circ. $2.5 \mathrm{~mm}$. longae margine late scariosae ciliolatae dorso plus minusve pilosae; flores $\hat{o}$ sessiles paria 3-4, sepala late elliptica apice acutiuscula membranacea scariosa circ. $2 \mathrm{~mm}$. longa dorso glabra, stamina sepalis circ. duplo longiora circ. $4 \mathrm{~mm}$. longa, antheris circ. 1.5 $\mathrm{mm}$. longis, ovarii rudimentum sepala fere aequans apice valde dilatatum tetragonum circ. $1.5 \mathrm{~mm}$. longum; flos o terminalis, sepala ovato-rotundata apice obtusa margine late scariosa dorso glabra circ. $2.5 \mathrm{~mm}$. longo, ovarium stylis apicem versus sensim plus minusve dilatatis paullo longiore glabrum, stigmata obcordata usque ad medium stylum decurrentia medio sulcata. Capsula ovoideo-globosa glabra lucida cum stylis persistentibus crassis valde

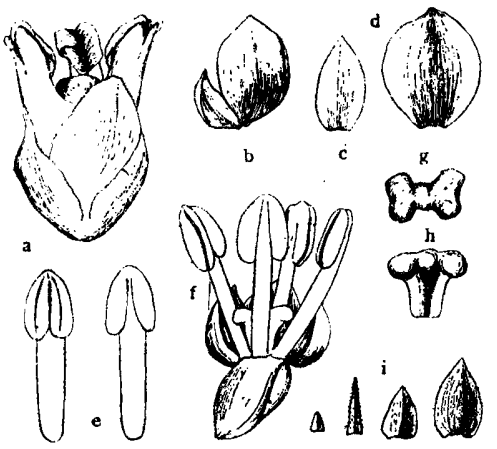

Fig. 26. Buxus microphylla subsp. sinica var. insularis Hatusima. a, female flower $\times 5$. b, sepals of male flower, seen from within $\times 4$. c, $d$, outer and inner sepals of male flower, seen from within $\times 4$. e, stamens, seen from different sides $\times 4.5$. $f$, male flower, showing rudimental ovary $\times 4.5$. $g, h$, rudimental ovaries, seen from above and side $\times 5$. $i$, bracts of different shapes, seen from without, magnified. 
divaricatis circ. $2.5 \mathrm{~mm}$. longis circ. $1 \mathrm{~cm}$. longa $6 \mathrm{~mm}$. lata ; semina trigono-ellipsoidea circ. $5 \mathrm{~mm}$. longa lucida nigerrima. Fig. 26, Pl. XI, Fig. 1.

Korea. Prov. Keiki: near Keijô, common on side of streams. on rotten granite base of Mt. Kwangaku-san, Nov. 23, 1917 (WILson,. no. 9625; type of var. koreana NAKAI ex REHDER, in HA); Mt. Nankan-zan, Sept. 23, 1918 (WiLson, no. 10742 ; in HA). Prov. Tyusei : Mt. Daihô-rei, common, Dec. 9, 1917, (WiLson, no. 9631; in HA). . Prov. Keihoku: Mt. Syûo-zan, Aug. 29, 1939 (S. Ito, no. 260)Prov. Keinan: Mt. Tizan, 1936 (R. KaneHIRA, no. 3776). Prov. Zennan: Isl. Kitihotô, July 3, 1936 (H. D. ChANG, no. 60); same island, April 28, 1936 (H. D. CHANG, no. 59); same island, without date (H. UYEKI). Quelpaert: in silvis Sampangsan, Oct. 1906 (TAUQET, no. 1379 ; in HA).

China. Kiangsi : Santuchüan, Lushan, in ravine, Aug. 17, 1934 (P. C. YEH, 468; shrub $30 \mathrm{~cm}$. ht.; in HA).

This variety is most closely related to the small-leaved form of subsp. sinica, from which it differs in the much thicker obscurely nerved leaves with strongly revoluted margines. I have not yet seen the original specimen of this variety which was collected on the island of Daikokuzan-tô, off the west coast of the Zennan province. But as far as I can judge by the several collections before me which were collected on the island of Kitiho-tô in the same province, this form agrees exactly with the Nakai's original description and seems to be an insular form which differs from the mainland form, generally known as var. koreana NAKAI, merely in the glabrescent branchlets with somewhat thicker and larger leaves apparently owing its origin to the moist warm-temperate climate of the island where it grows. However, degree of pubescence of the branchlets as well as the shape and size of the leaves are very variable and intermediate forms are not uncommon, and I believe that these differences are too slight to warrent even varietal delimitation. TAQUET, no. 1379 from Quelpaert cited above may represent a form of these intermediate forms.

REHDER, when describing var. koreana NAKAI overlooked the fact that Dr. NAKAI has already given a name to the Korean form two years before. 
Undermined and excluded species

Buxus chinensis LiNk, Enum. Hort. Berol. 2: 386 (1822)= Simmondsia chinensis (LINK) ScHNEIDER, Illus. Handb. Laubholzk. 2: 141, fig. 90 (1907).

Buxus Fortunei CARRIÈRE in Rev. Hort. 42: 519 (1870); Gard. Chron. 1615 (1871).

Buxus Fortunei CARR., sometimes known as Buxus longifolia Hort., which, according to the original description, must be a Chinese origin, has hitherto been reduced to a synonym of Buxus longifolia BoIsS. from Syria (SCHNEIDER, REHDER and WILSON, etc.) or Buxus Harlandii HANCE from Hongkong. The original description is very incomplete and reads in part: "Ses feuilles, en effet, subcunéiformes, sont relativement longues et étroites......... L'espèce chinoise Buxus Fortunei, CARR., forme un buisson trèscompact, à branches nombreuses, strictement dressées, à feuilles très-rapprochées, légèrement étalées. Cette plante, qui est extrêmement rustique, suporte les hivers les plus rigoureux du climat de Paris." Judging from the above description and specimens distributed to the herbarium of the Arnold Arboretum by C. K. SCHNEIDER under the name Buxus Fortunei CARR. and by Nicolson under the name Buxus Harlandii HANCE, both of which were taken from the cultivated plants in Europe, Buxus Fortunei CARR. does not seem to differ from Buxus Bodinieri LÉvL. from western Cnina, and I am now inclined to believe that Buxus Fortunei CARR. may be an oldest name for the Léveillean species. However, since in the absence of the type specimen it seems impossible to determine the identity of the plants to which the name Buxus Fortunei should properly be restricted and it seems advisable to abondon the name Buxus Fortunei and the next available name, Buxus Bodinieri, should be used for this plant. 


\section{LITERATURE}

BatlzoN, H.

(1) Monographie des Buxacées et des Stylocecées 58-63 (1859).

Benthas, G. (2) Flora Hongkonensis 315 (1861).

Bentham, G. \& Hooker, J. D.

(3) Genera Plantarum 3: 266 (1880).

CARRIÈrE, E. A. (4) Revue Horticole 519-520 (1870).

DippLL, L. (5). Handbuch der Laubholzkunde 3: 79-83 (1893).

DunN, S. T. \& TLchTer, W. J.

(6) Flora of Kwangtung and Hongkong in the Kew Bulletin of Miscellaneous Information add. ser. 10, 233 (1912).

EI,wes, H. J. \& HeNRY, A.

(7) The Trees of Great Britain \& Ireland 7: 1719-1730 (1913).

Franchet, $\mathbf{A}$.

(8) Plantae Davidianae ex Sinarum Imperio 1 Partie, Plantes, du Thibet Orientale 136 (1889).

Franchet, A. \& Savatier, L.

(9) Enumeratio Plantarum in Japonica sponte Crescentium 1: 428 (1875).

Gagnepain, F.

(10) In Lecomte, Flore Genérale de L'indo-Chine 5: 660-666 (1927).

(11) Buxus in Bulletin de la Société botanique de France 68: 482 (1921).

Gamble, J. S.

(12) A Manual of Indian Timbers 592-593 (1902).

Hanct, H. F.

(13) Buxus in Sermann's Jonrnal of Botany 6: 331 (1868).

(14) Buxus in Journal of the Linnean Society. Botany 13: 123-124 (1873)

Handel-MazzEttr, H. (15) Symbolae Sinicae, Botanische Ergebnisse der Expedition der Akademie der Wissenschaften in Wien nach Südwest China 7: 236-237 (1931).

HaYATA, B.

(16) Revisio Euphorbiacearum et Buxacearum in Journal of the College of Science of Imperial University of Tokyo 23: art. 10, 82-84 (1908).

HemsLi:y, W. B.

(17) Buxus in Journal of the Linnean Society, Botany 26: 418 (1894).

HOOKER, J. D.

(18) Flora of British India 5: 267 (1887).

KANEHIRA, $\mathbf{R}$.

(19) Formosan Trees rev. ed. 359 (1936).

KOEHNE, E.

(20) Deutsche Dendrologie 356 (1893).

Líverití, $\mathrm{H}$.

(21) Buxus in FEdvs, Repatorium Novarum Specierum Regni Vegetabilis 11: 549 (1913).

(22) Flore du Kouy-Tchéou 160 (1914).

LÉVELLL'́, H. \& Vaniot, E.

(23) Buxus in FEdde, Repatorium Novarum Specierum Regni Vegetabilis 3: 20 (1906). 
MaKino, T.

(24) Buxus in Botanical Magazine Tokyo 9 : 279-280 (1895); 15: 169 (1901); 16: 179 (1902); 26: 293 (1912); 27 : 112-113 (1913).

(25) Buxus in Zitssai-engei 15: 529 (1939).

MaKino, T. \& Nemoto, K.

(26) Flora of Japan ed. 2, 633-634 (1931).

Muetrer-Arg.

(27) In DE CANDoL LE, Prodromus Systematis Naturalis Regni Vegetabilis 16: 13-20 (1869).

MAYR, H.

(28) Fremlandische Wald- und Parkbäume für Europa 451 (1906).

Merrill, E. D.

(29) Buxus in Philippine Journal of Science, Botany 1: 84 (1906); 9: 309-310 (1914).

(30) Buxus in Lingnan Science Journal 14: 25 (1935).

(31) An Enumeration of Philippine Flowering Plants 2: 464-465 (1923).

PAX, F.

(32) Buxus in Engler \& Frantl, Die Natürlichen Pflanzenfamilien 3 (5): 130 (1890).

ReHDER, A. \& WiLSON, E. H.

(33) In Sargent, Plantae Wilsonianae 2: 165-169 (1914).

REHDER, A.

(34) Buxus in Journal of the Arnold Arboretum 7: 240 (1926); $14: 236$ (1933); 18 : 215 (1937).

RidLEY, H. N.

(35) Manual of Cultivated Trees \& Shrubs 532-533 (1927).

(36) The Flora of the Malay Peninsula 3: 182 (1924).

(37) Buxus in Journal of the Straits Branch of the Royal Asiatic Society 59: 166-167 (1911).

(38) Buxus in Kew Buttetin of Miscellaneous Information 475 (1926).

Schneider, C. K.

(39) Illustriertes Handbuch der Laubholzkunde 2: 137-140 (1907).

SieboLd, P. F.

(40) Synopsis Plantarum Oeconomicarum Universi Regni Japonici 30 (1830).

Sмiтh, W. W.

(41) Buxus in Note from the Royal Botanic Garden Edinburgh 9 : 88 (1916); $10: 16$ (1917).

Thun Blirg, C. P.

(42) Flora Japonica 77 (1784). 


\section{INDEX}

Synonyms are printed in ltalics; new names in bold-faco type.

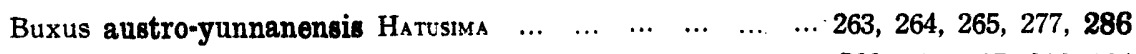
Bodinieri LÉvL. $\quad \ldots \quad \ldots \quad \ldots \quad \ldots \quad \ldots \quad \ldots 263,265,266,280,311,314,315,316,334$

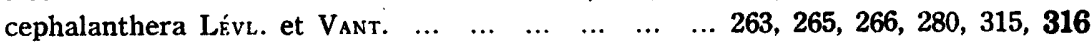
$\begin{array}{llllllllllllllllll}\text { chinensis } \operatorname{LiNK} \ldots & \ldots & \ldots & \ldots & \ldots & \ldots & \ldots & \ldots & \ldots & \ldots & \ldots & \ldots & \ldots & \ldots & \ldots & \ldots & 334\end{array}$ $\begin{array}{llllllllllllll}\text { cochinchinensis PIERRE: } & \ldots & \ldots & \ldots & \ldots & \ldots & \ldots & \ldots & \ldots & \ldots & \ldots & \ldots & \ldots & 268,271\end{array}$

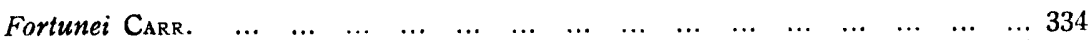
hainanensis MERR ...

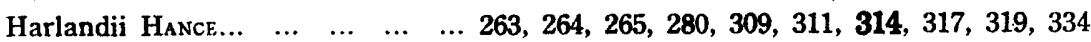

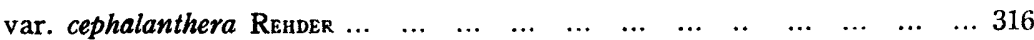

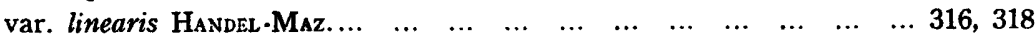

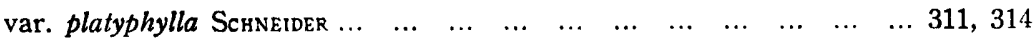

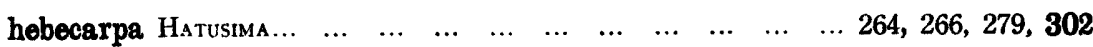
$\begin{array}{llllllllll}\text { Henryi } M_{\text {AYR }} \ldots & \ldots & \ldots & \ldots & \ldots & \ldots & \ldots & \ldots & \ldots & 263,264,265,278,285,296,299\end{array}$

Holttumiana Hatusima ichangensis HatuSIMA var. fukienensis Hatusima $\begin{array}{lllllllllllllll}\text { intermedia } \mathrm{K}_{\mathrm{ANEHIRA}} & \ldots & \ldots & \ldots & \ldots & \ldots & \ldots & \ldots & \ldots & \ldots & \ldots & \ldots & \ldots & \ldots & 291,326\end{array}$ $\begin{array}{lllllllllllllll}\text { japonica MUELLER-ARg. } & \ldots & \ldots & \ldots & \ldots & \ldots & \ldots & \ldots & \ldots & \ldots & \ldots & \ldots & \ldots & 321,331\end{array}$ $\begin{array}{lllllllllllllll}\text { f. } r \text { rubra } \text { MAKIN }_{1} & \ldots & \ldots & \ldots & \ldots & \ldots & \ldots & \ldots & \ldots & \ldots & \ldots & \ldots & \ldots & 324,325\end{array}$

$\begin{array}{lllllllllllllll}\text { a microphylla Muglder-Arg. } & \ldots & \ldots & \ldots & \ldots & \ldots & \ldots & \ldots & \ldots & \ldots & \ldots & \ldots & 320\end{array}$ $\begin{array}{llllllllllllllll}\text { var. variegata } \text { Dippli. } & \ldots & \ldots & \ldots & \ldots & \ldots & \ldots & \ldots & \ldots & \ldots & \ldots & \ldots & \ldots & \ldots & 325\end{array}$

$\begin{array}{llllllllllllllll}\text { Kitashimai } \mathrm{Y}_{\mathrm{ANAGIDA}} & \ldots & \ldots & \ldots & \ldots & \ldots & \ldots & \ldots & \ldots & \ldots & \ldots & \ldots & \ldots & \ldots & \ldots & 325\end{array}$

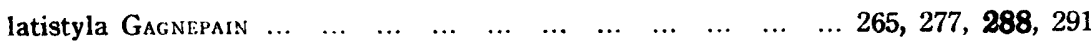

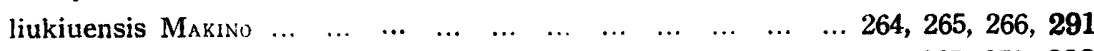
var. longipedicellata Hatusima longifolia BALLION...

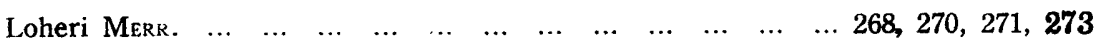
$\begin{array}{lllllllllllll}\text { malayana RidLIYY } & \ldots & \ldots & \ldots & \ldots & \ldots & \ldots & \ldots & \ldots & \ldots & \ldots & \ldots & 265,278,286,289\end{array}$ $\begin{array}{lllllllllll}\text { megistophylla Lévl. } & \ldots & \ldots & \ldots & \ldots & \ldots & \ldots & \ldots & \ldots & \ldots & 264,265,284,295,297\end{array}$

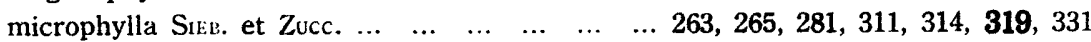

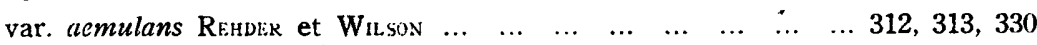
$\begin{array}{llllllllllllll}\text { var. arborescens } \mathrm{N}_{\mathrm{AKAI}} \ldots & \ldots & \ldots & \ldots & \ldots & \ldots & \ldots & \ldots & \ldots & \ldots & \ldots & \ldots & \ldots & 322\end{array}$ $\begin{array}{lllllllllllllll}\text { var. insularis } \mathrm{N}_{\mathrm{AKAI}} & \ldots & \ldots & \ldots & \ldots & \ldots & \ldots & \ldots & \ldots & \ldots & \ldots & \ldots & \ldots & \ldots & 331\end{array}$

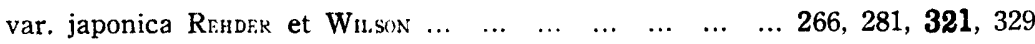

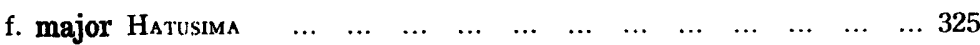

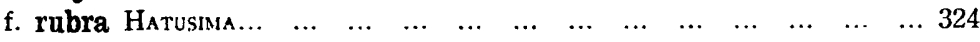

$\begin{array}{lllllllllllllll}\text { var. koreana } \mathrm{N}_{\mathrm{AKAI}} & \ldots & \ldots & \ldots & \ldots & \ldots & \ldots & \ldots & \ldots & \ldots & \ldots & \ldots & \ldots & \ldots & 332\end{array}$

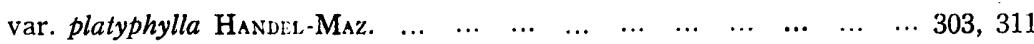
$\begin{array}{lllllllllllllll}\text { var. prostrata } \mathrm{Sм} \mathrm{\text {мттн }} & \ldots & \ldots & \ldots & \ldots & \ldots & \ldots & \ldots & \ldots & \ldots & \ldots & \ldots & \ldots & \ldots & 306\end{array}$

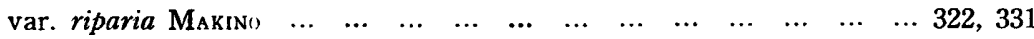

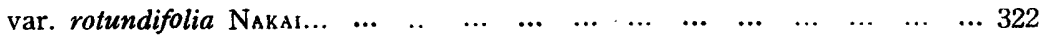

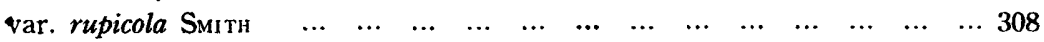
var. sinica R RHDER et WILSON... 
$\begin{array}{lllllllllllllll}\text { var. suffruticosa. MAKINO } & \ldots & \ldots & \ldots & \ldots & \ldots & \ldots & \ldots & \ldots & \ldots & \ldots & \ldots & \ldots & 322\end{array}$

f. gigantea MAkino...

f. minutissima MaKiNo...

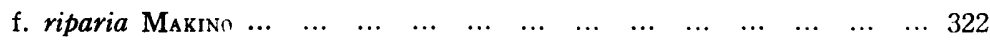

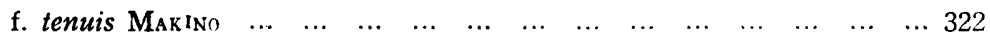

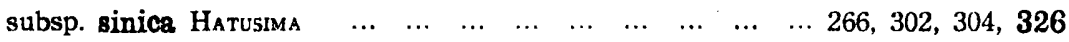

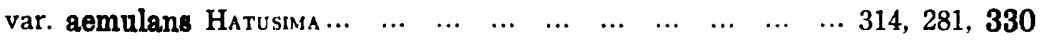

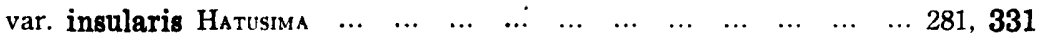

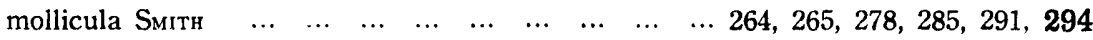

var. glabra HAN DEL-MAZ...

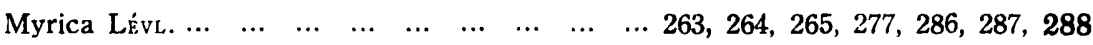

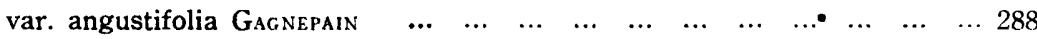

$\begin{array}{lllllllllllllll}\text { pachyphylla MERR. } & \ldots & \ldots & \ldots & \ldots & \ldots & \ldots & \ldots & \ldots & \ldots & \ldots & \ldots & \ldots & \ldots & 268,269\end{array}$

papillosa Schnerder $\quad \ldots \quad \ldots \quad \ldots \quad \ldots \quad \ldots \quad \ldots \quad \ldots \quad$ 263, 264, 265, 265, 278, 283, 298

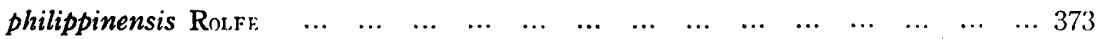

viparia MAKINO

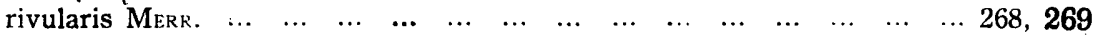

Rolfei VIDAL $\quad \ldots \quad \ldots \quad \ldots \quad \ldots \quad \ldots \quad \ldots \quad \ldots \quad \ldots \quad \ldots \quad 267,268,269,270,271,272,274$

rugulosa Hatusima $\quad \ldots \quad \ldots \quad \ldots \quad \ldots \quad \ldots \quad \ldots 263,264,265,266,279,301,303,330$

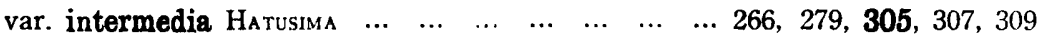

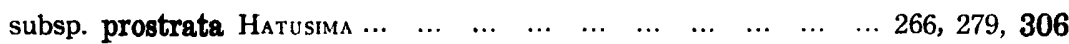

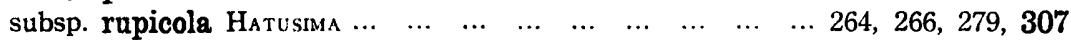

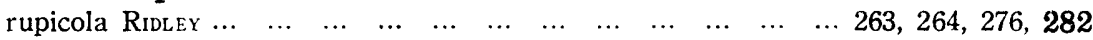

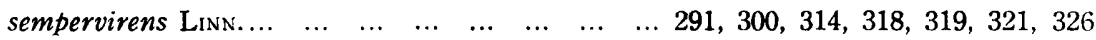

$\begin{array}{llllllllllllllll}\alpha \text {, angustifolia } \mathrm{S}_{\mathrm{IEB}} . & \ldots & \ldots & \ldots & \ldots & \ldots & \ldots & \ldots & \ldots & \ldots & \ldots & \ldots & \ldots & \ldots & 320\end{array}$

var. japonica MAKINo

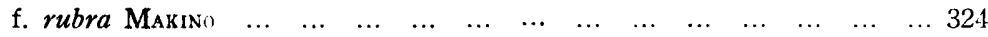

$\begin{array}{llllllllllllll}\text { var. liukiuensis MaKino } & \ldots & \ldots & \ldots & \ldots & \ldots & \ldots & \ldots & \ldots & \ldots & \ldots & \ldots & \ldots & 291\end{array}$

$\begin{array}{lllllllllll}\alpha \text { microphylla BLUMe } \ldots & \ldots & \ldots & \ldots & \ldots & \ldots & \ldots & \ldots & \ldots & \ldots & 306,307,316,319\end{array}$

$\begin{array}{lllllllllllllll}\text { var. riparia MAKiNO } & \ldots & \ldots & \ldots & \ldots & \ldots & \ldots & \ldots & \ldots & \ldots & \ldots & \ldots & \ldots & \ldots & 322\end{array}$

$\begin{array}{llllllllllllllll}\alpha \text { suffruticosa } \operatorname{Sin} . \ldots & \ldots & \ldots & \ldots & \ldots & \ldots & \ldots & \ldots & \ldots & \ldots & \ldots & \ldots & \ldots & \ldots & 321\end{array}$

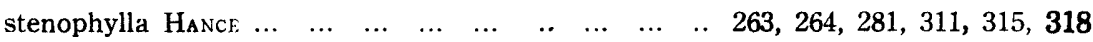

Wallichina Baillon $\ldots . \ldots . \ldots .263,264,265,266,279,291,300,302,303,305,331$

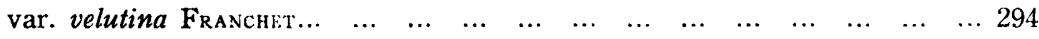

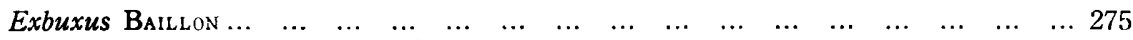

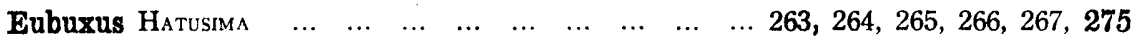

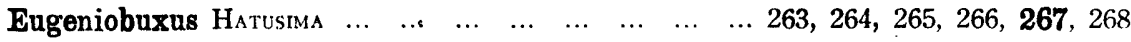

Simmondsia chinensis Schneider 


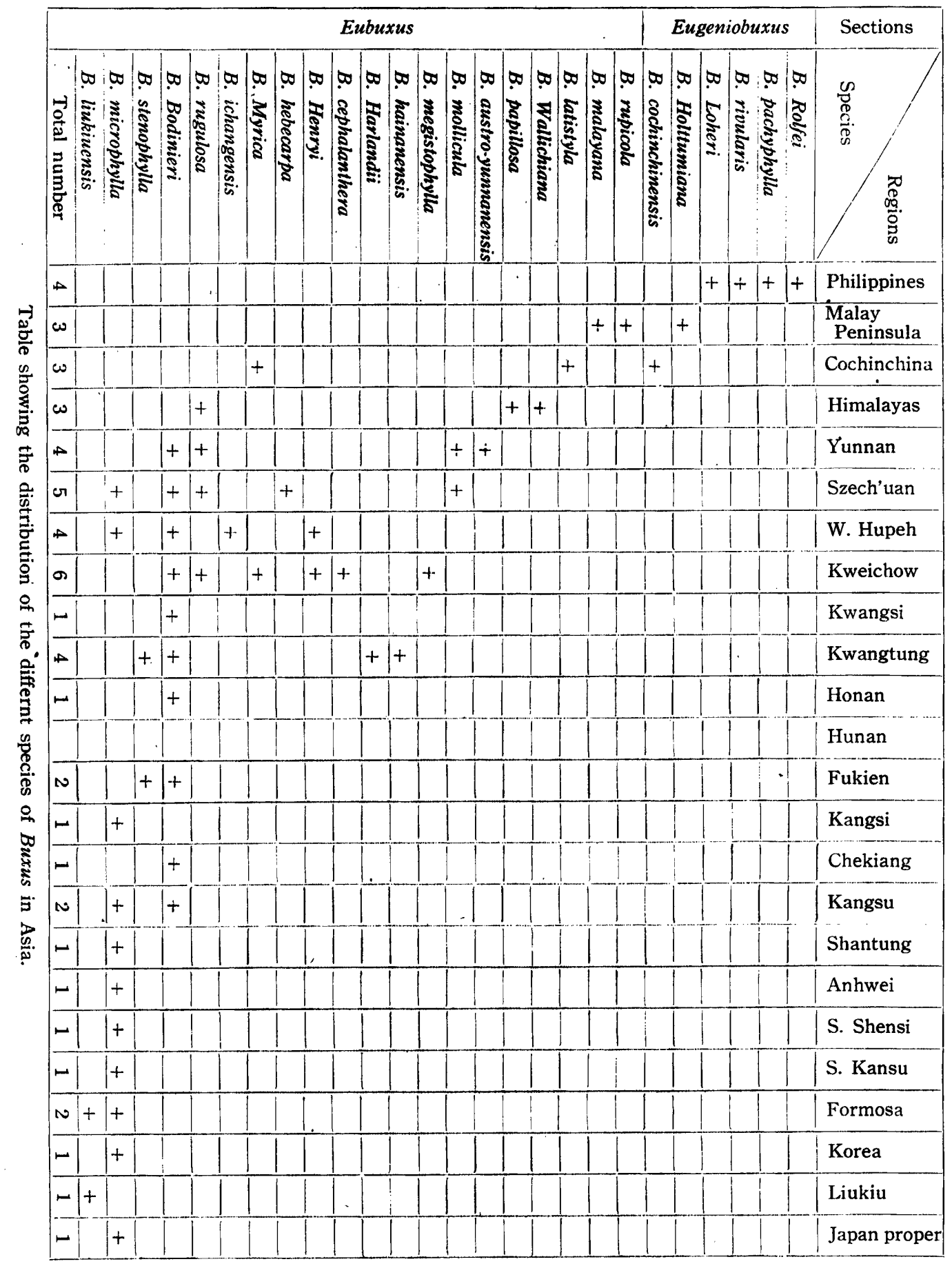




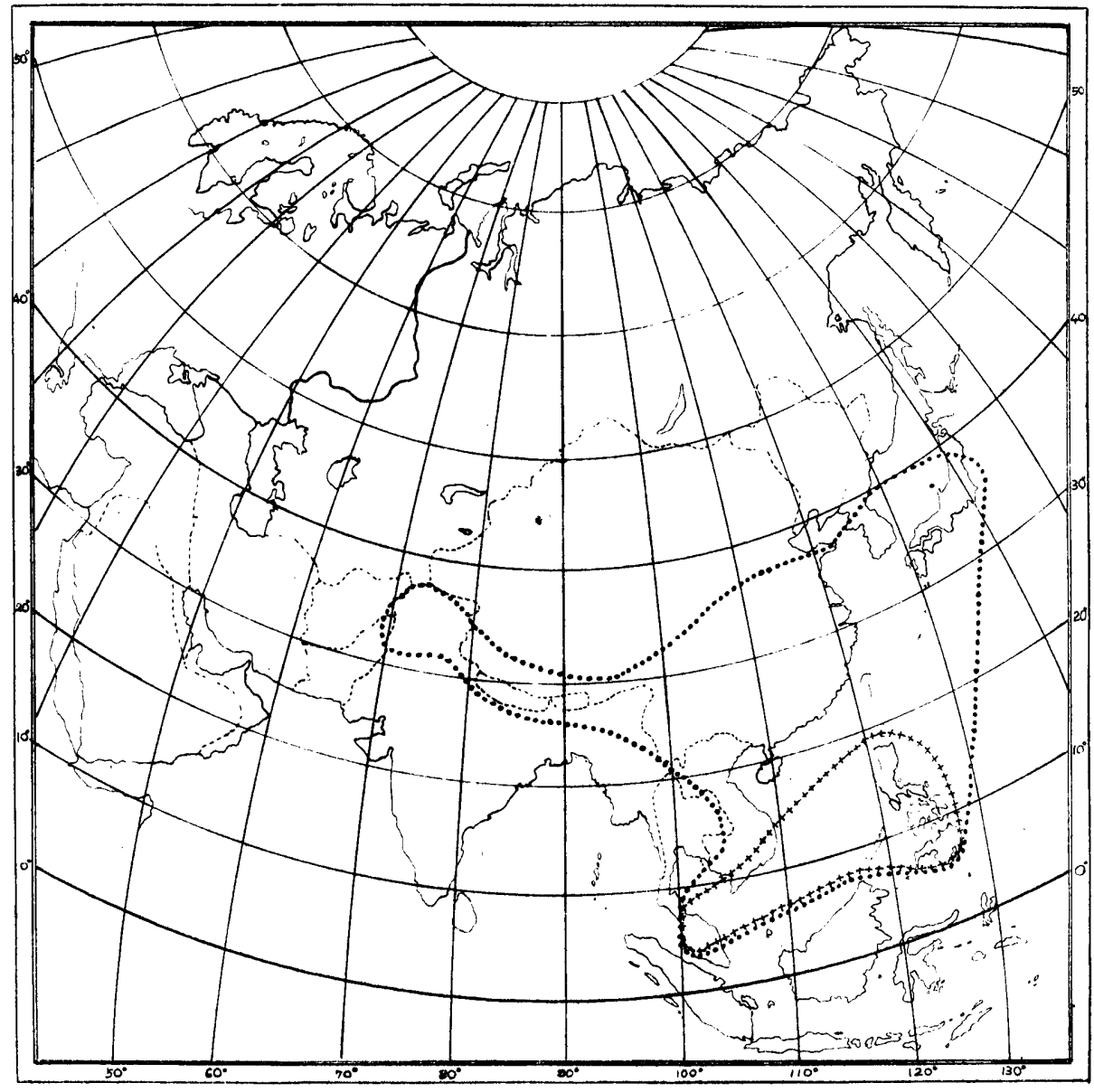

Map. 1. Map showing the range of $\dot{B} u x u s(\cdots . .$.$) and the Section Eugeniobuxus (+++++)$ in Asia. 


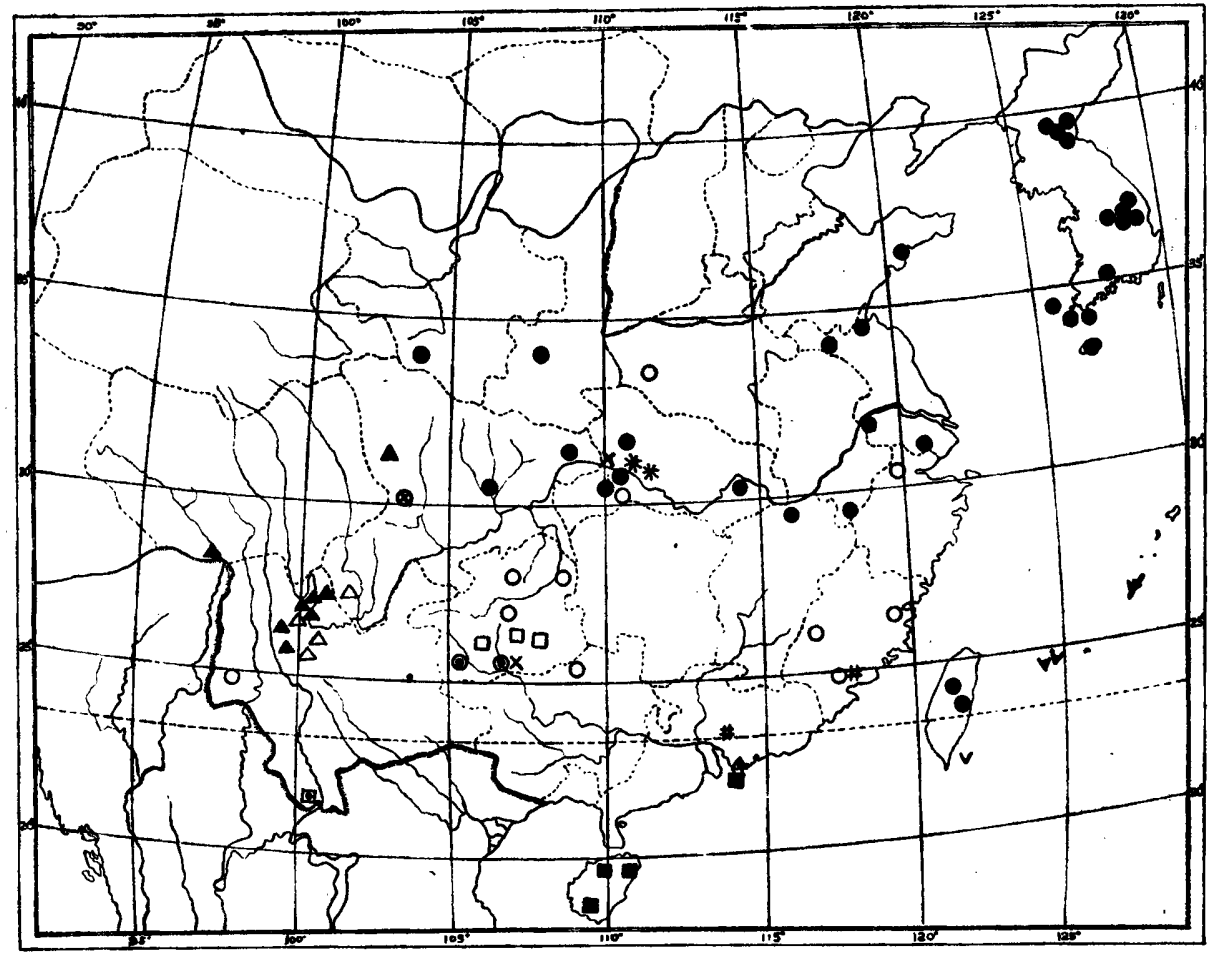

Map 2. Map showing the distribution of the different species of Buxus in China and adjacent districts.

B. microphylla subsp. sinica, together with its varieties.

A B. rugulosa, together with its subspecies and varieties.

O B. Bodinieri. $\square$ B. cephalanthera. $\times$ B. Henryi. $\quad *$ B. ichangensis.

○ B. Myrica. $\square$ B. hainanensis. $\triangle$ B. Harlandii. \# B. sienophylla.

$\vee$ B. liukiuensis. $\otimes$ B. hebecarpa. $\triangle$ B. molliculla. $\square$ B. austro-yunnanensis. 


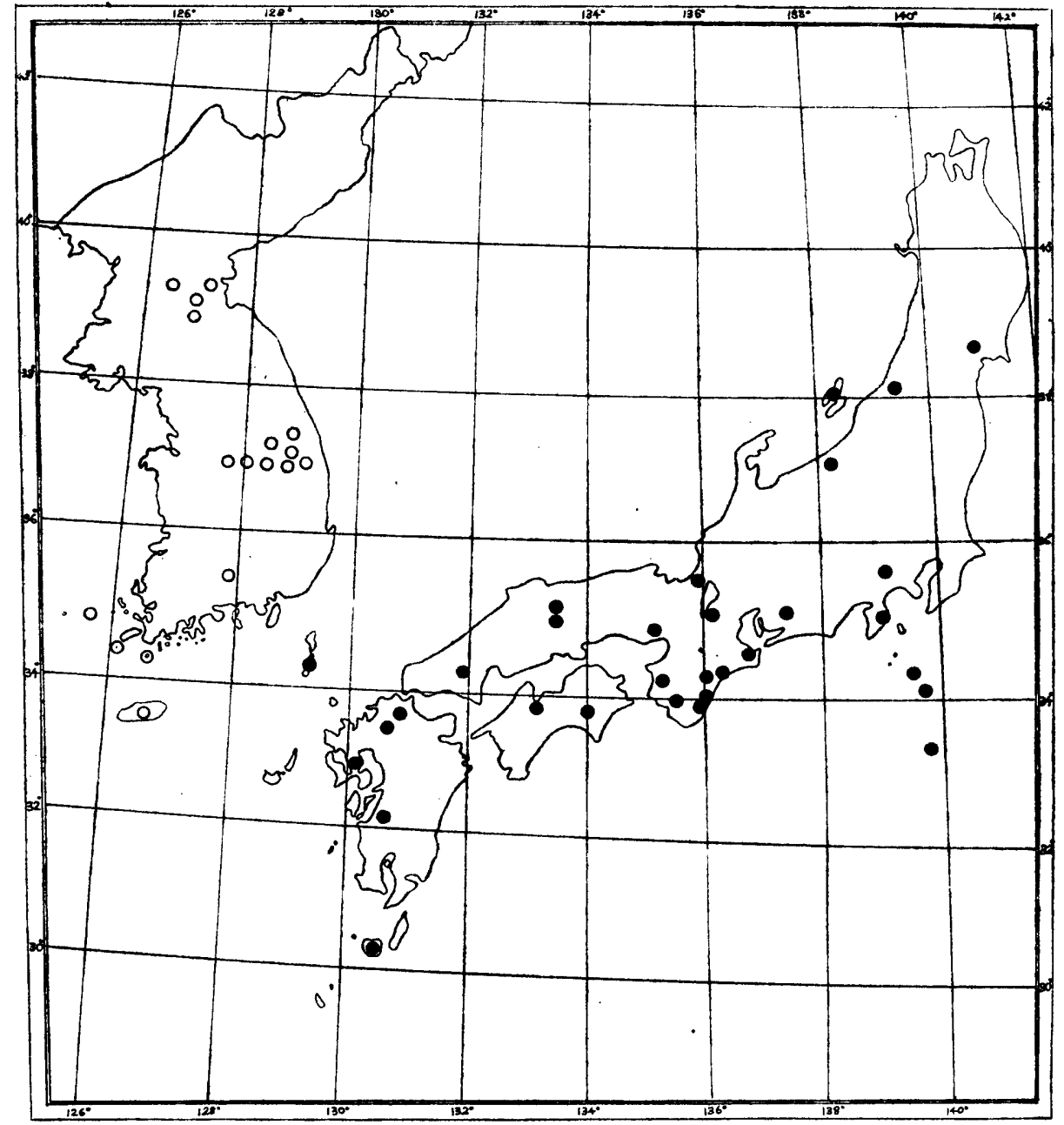

Map 3. Map showing the distribution of species of Buxus in Japan proper and Korea.

- B. microphylla var. japonica.

( B. microphylla var. japonica f. major.

O B. microphylla subsp. sinica var. insularis. 


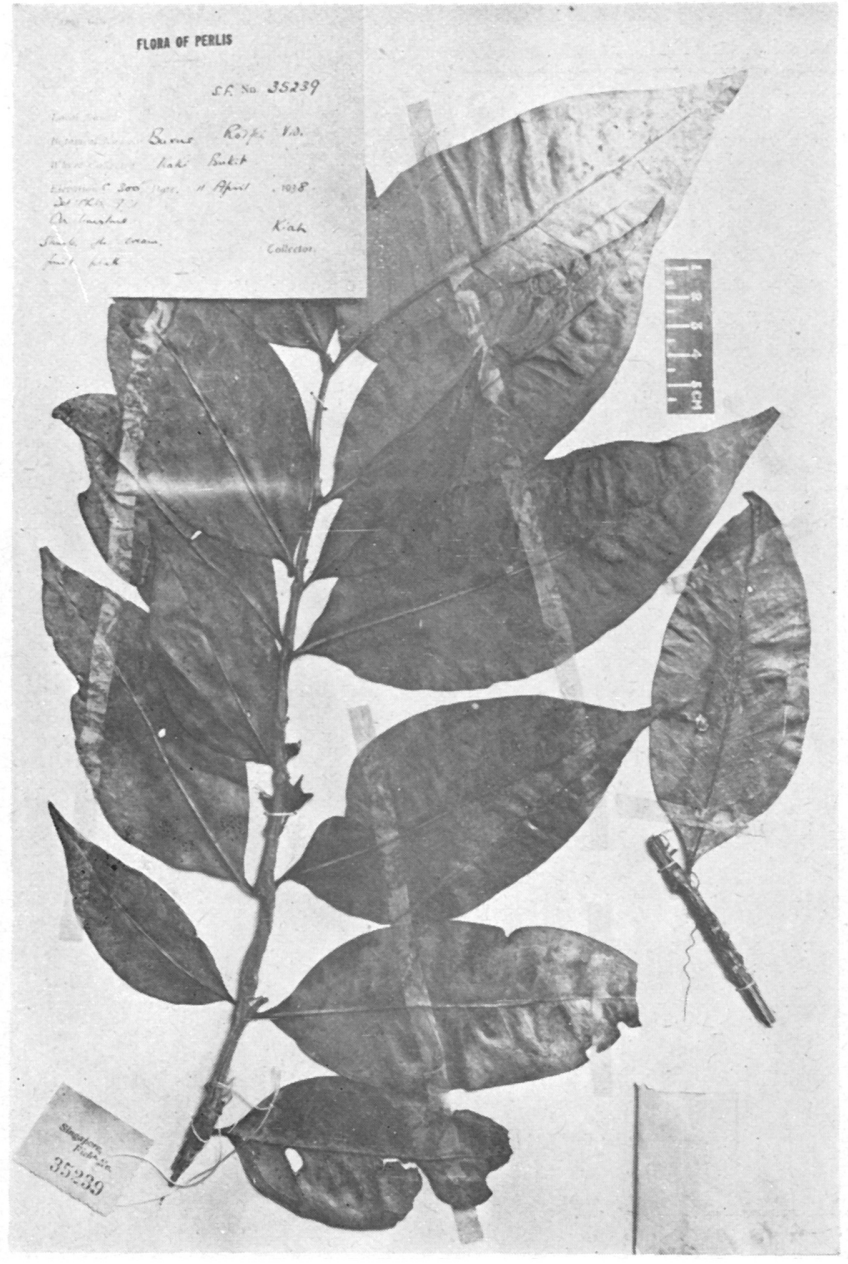

Fig. 1. Buxus Holtumiana Hatusima (type).

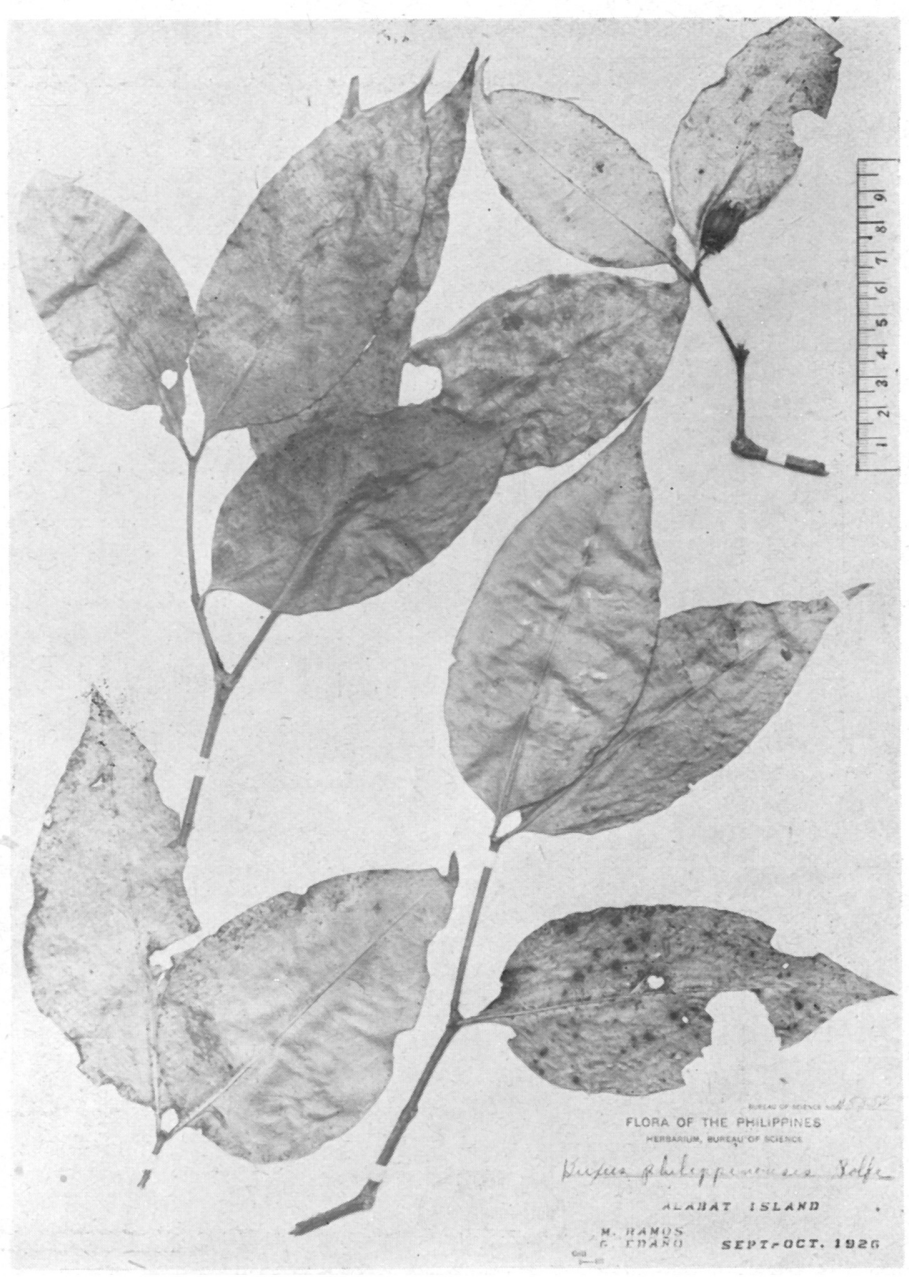

Fig. 2. Buxus Rolfei VIDaL, from Alabat Island.

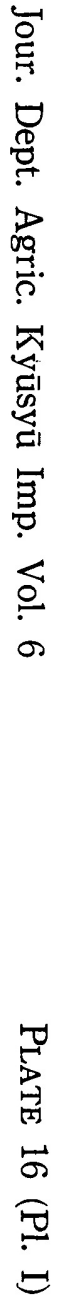

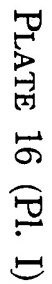




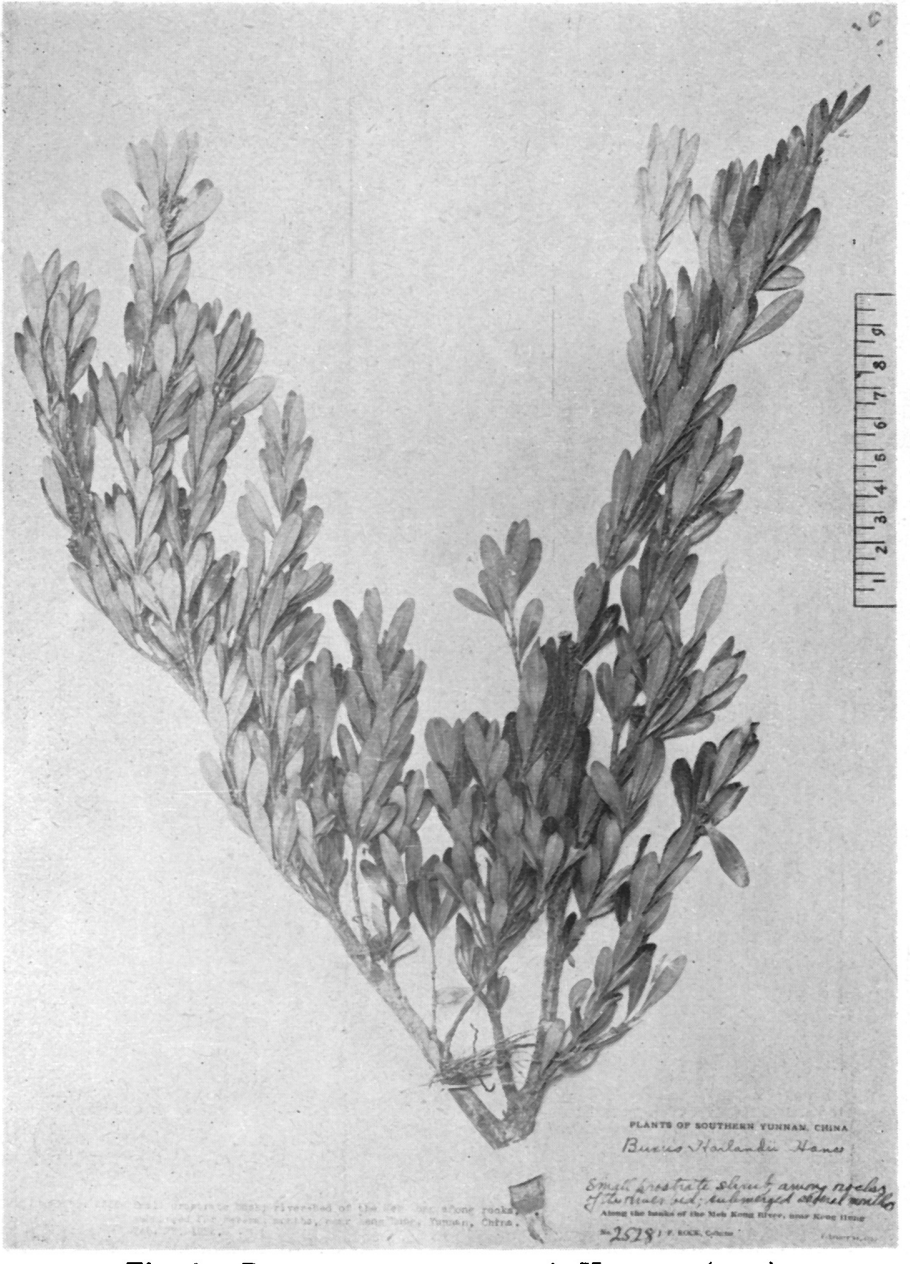

Fig. 1. Buxus austro-yunnanensis Hatusima (type).

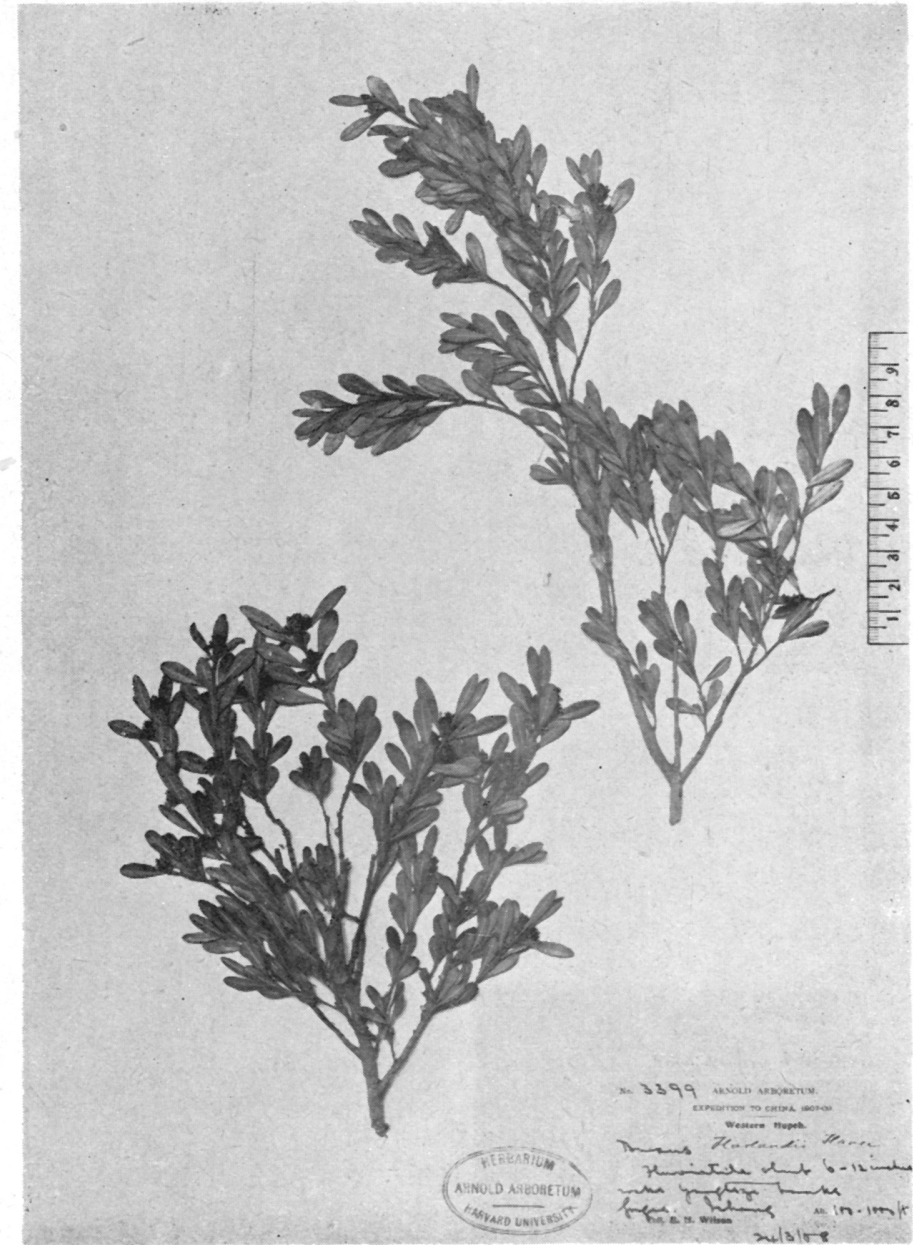

Fig. 2. Buxus ichangensis Hatusima (type).

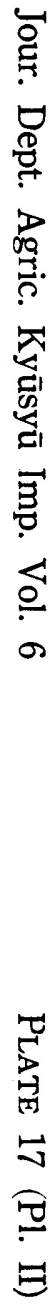

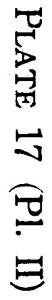




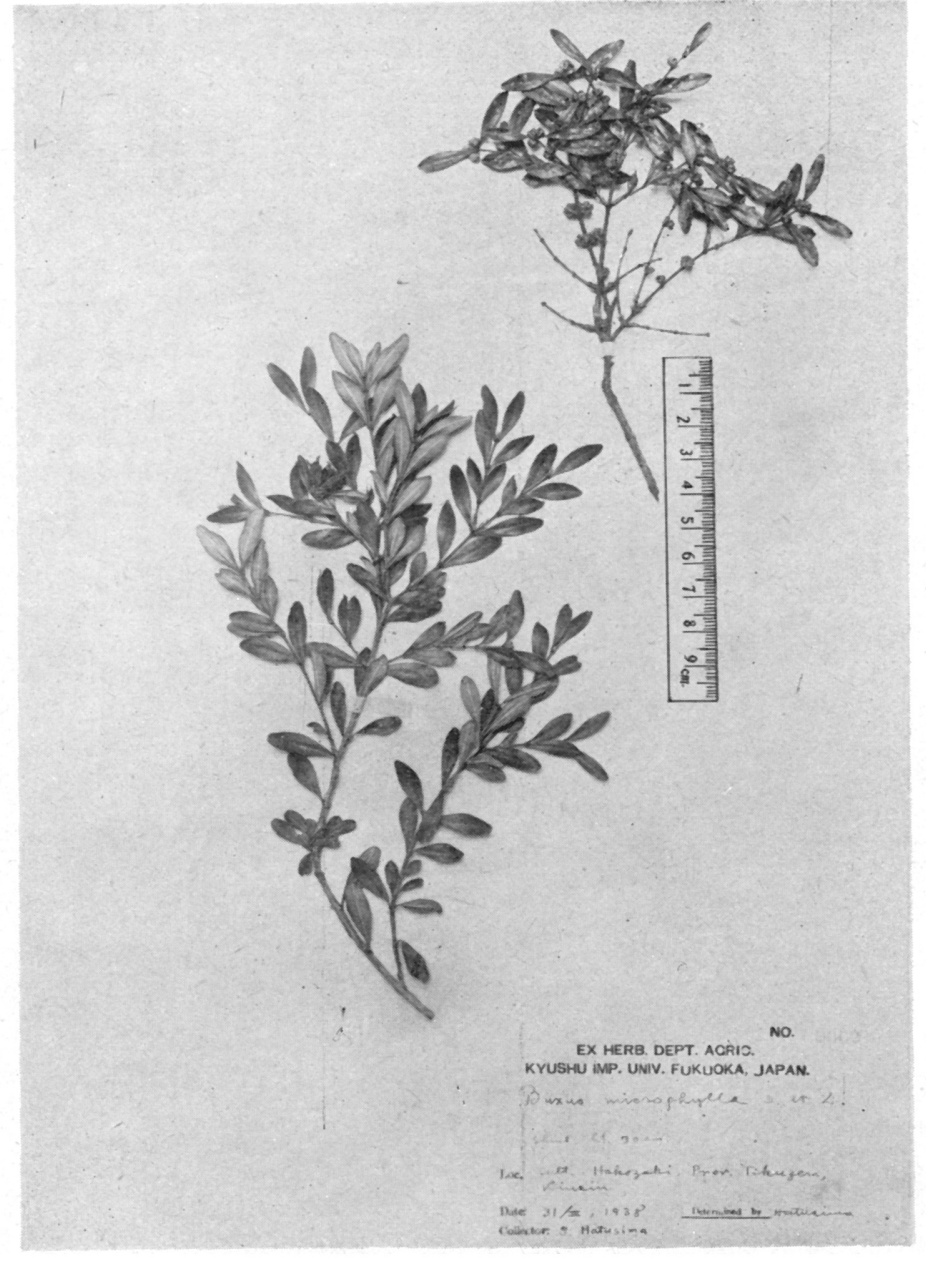

Fig. 1. Buxus microphylla Sirb. et Zucc.

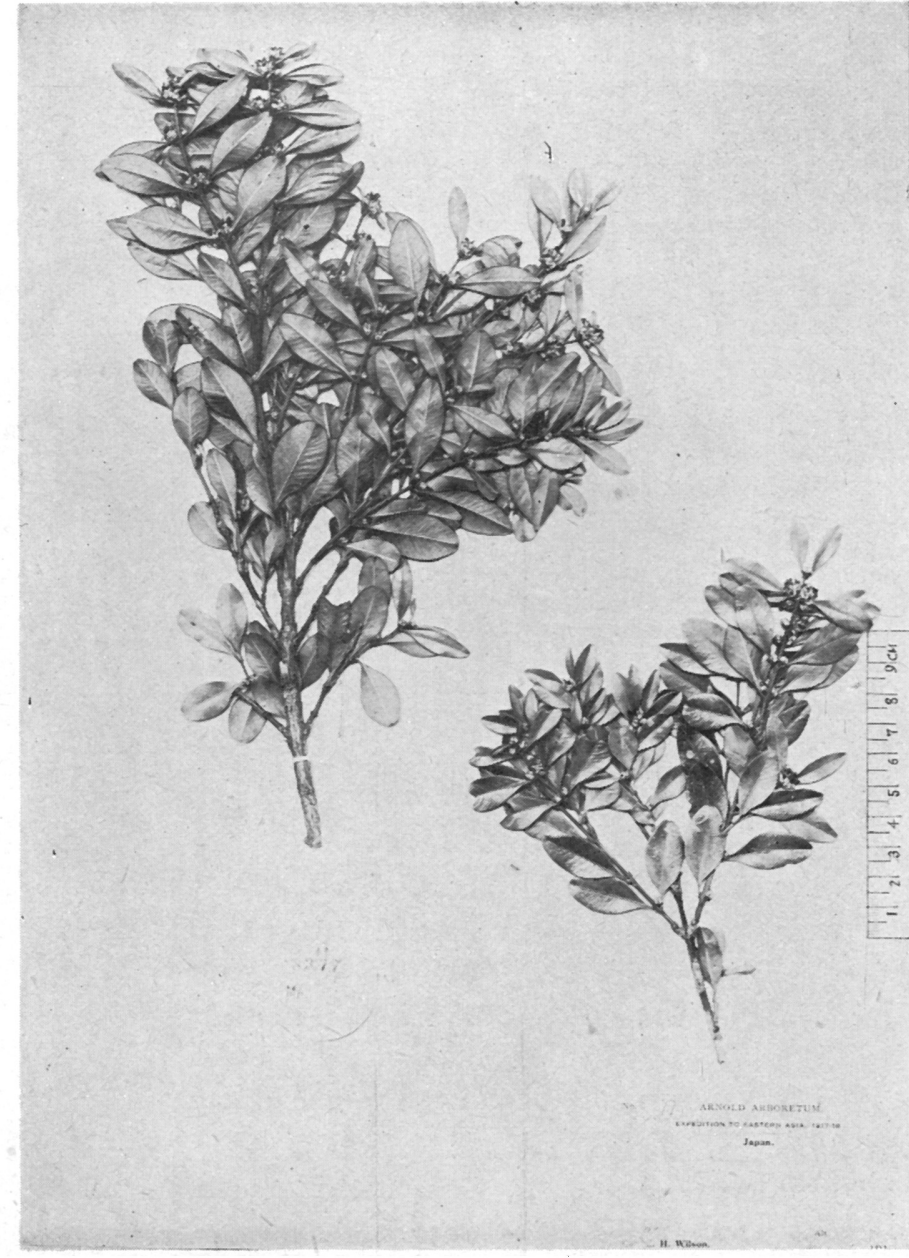

Fig. 2. Buxus microphylla var. japonica form. major Hatusima (WILSON, no. 8377).

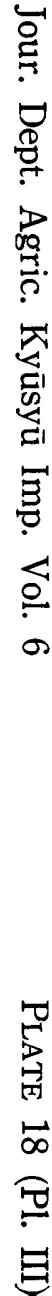




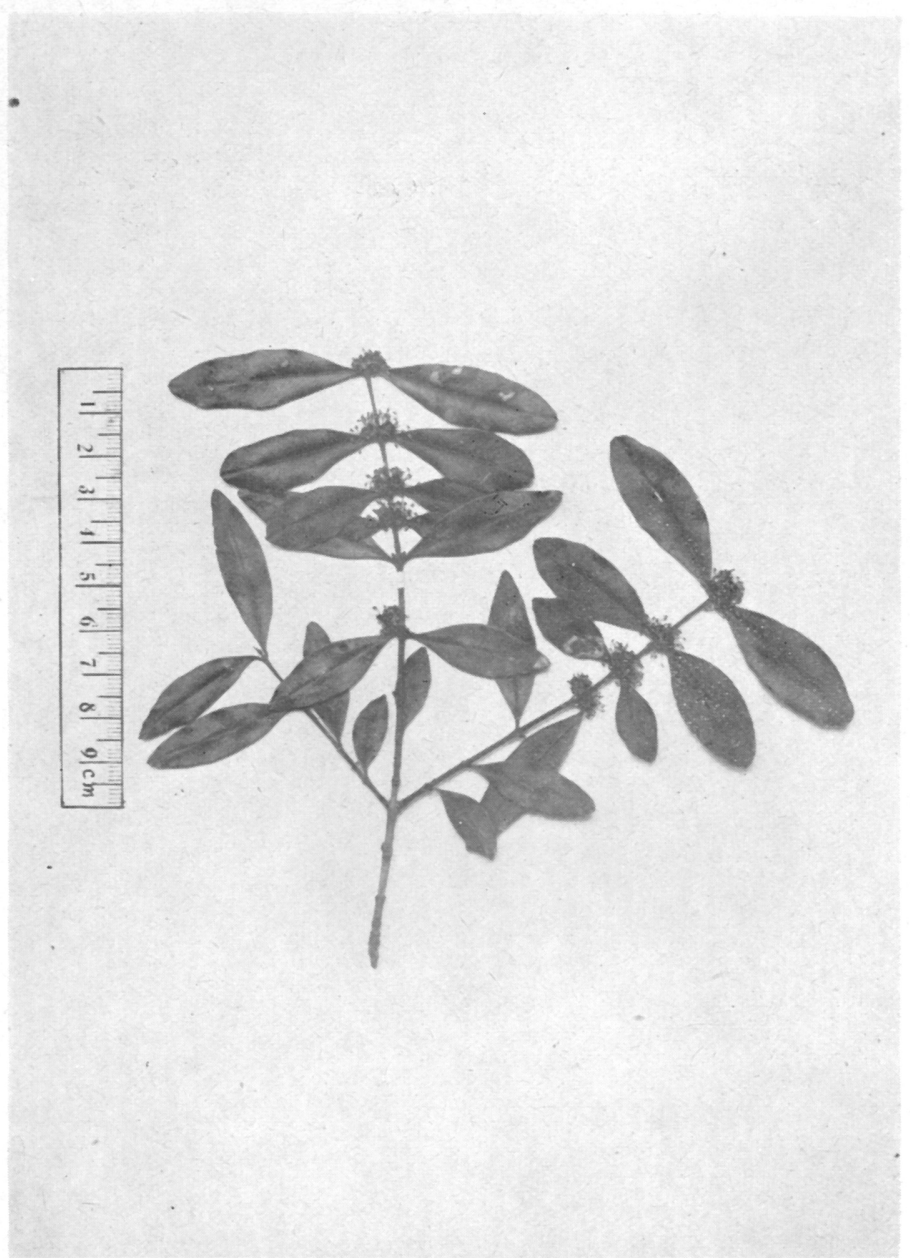

Fig. 1. Buxus Bodinieri LévéILle (type:

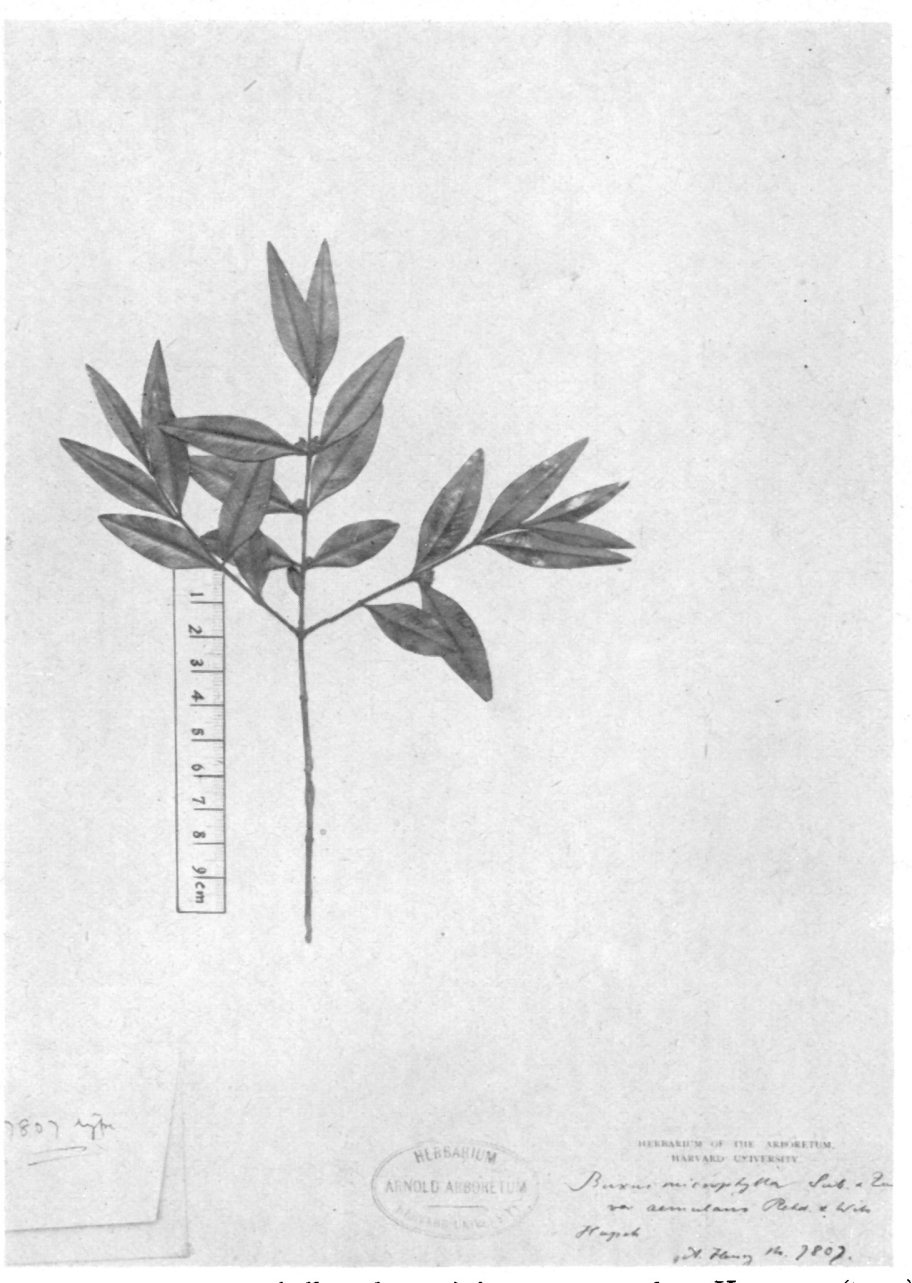

Fig. 2. Buxus microphylla subsp. sinica var. cemulans Hatusima (type).

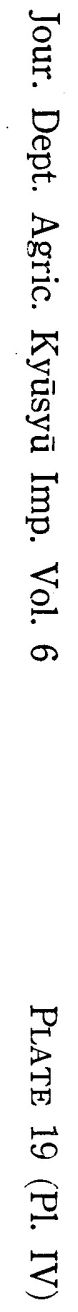




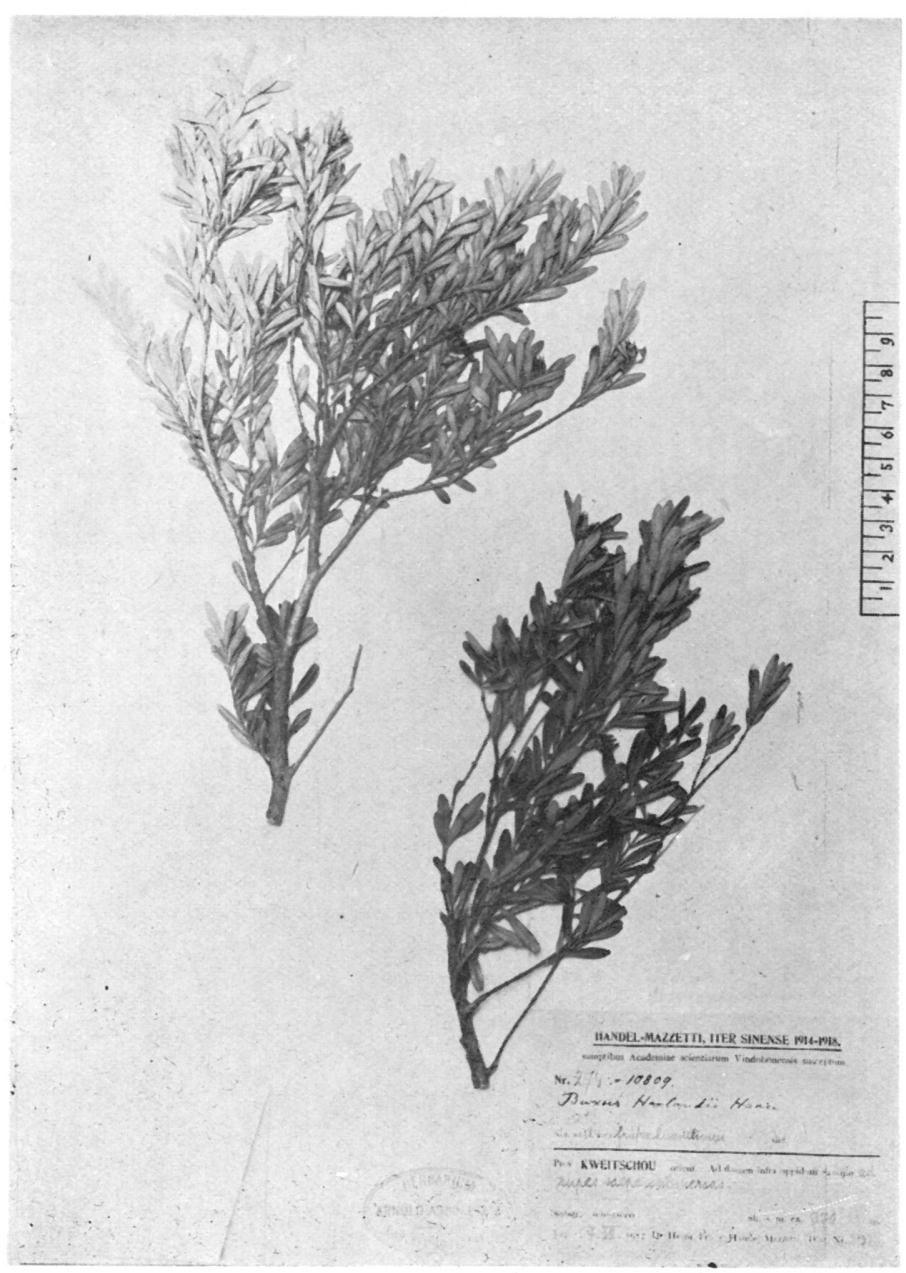

Fig. 1. Buxus cephalanthera LÉveILLÉ (type of B. Harlandii var. linearis HANDEL-MAZzETTI).

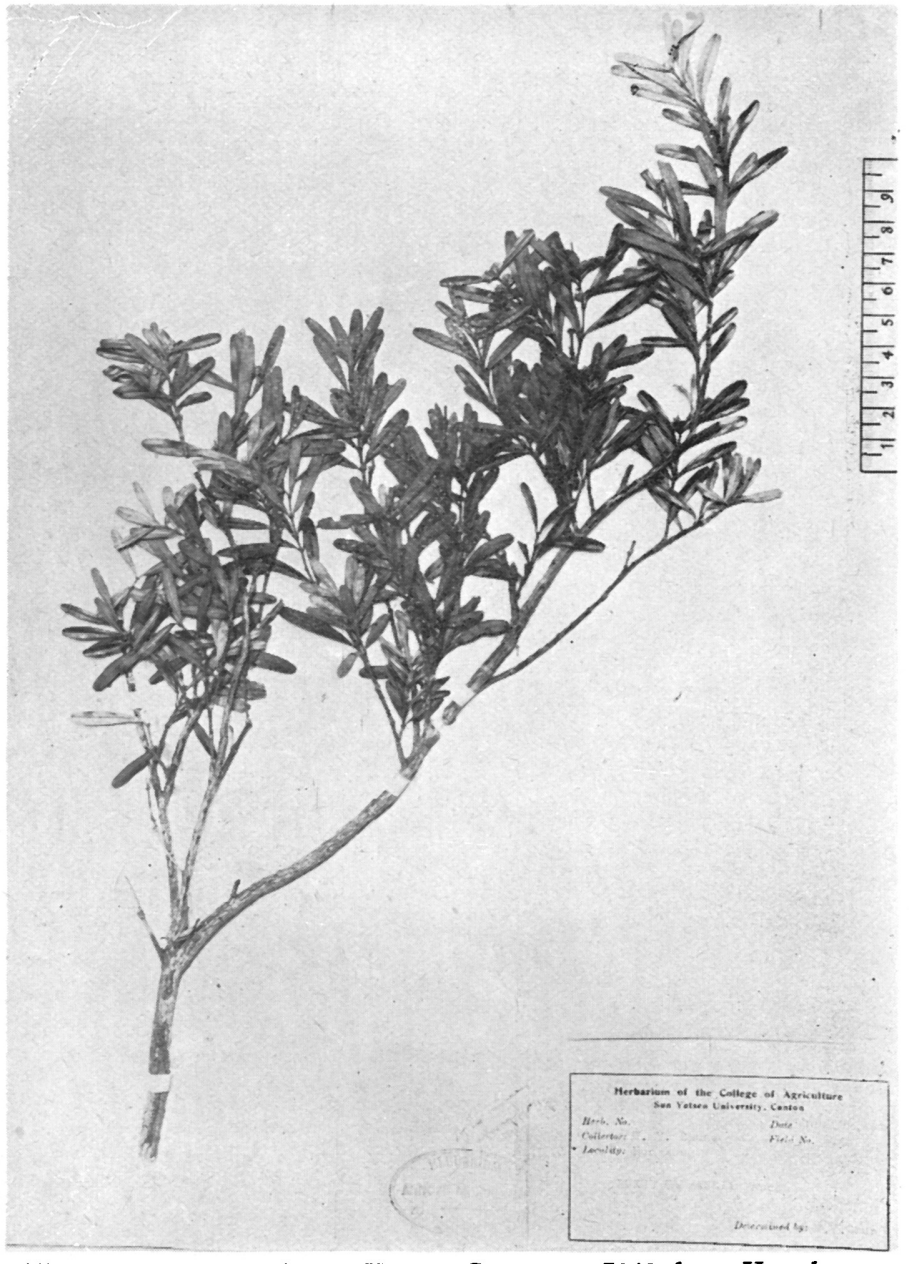

Fig. 2. Buxus Harlandii HaNCE (CHUN, no. 5040 from Hongkong; leaves somewhat narrower than the type).

里 $\zeta$ 


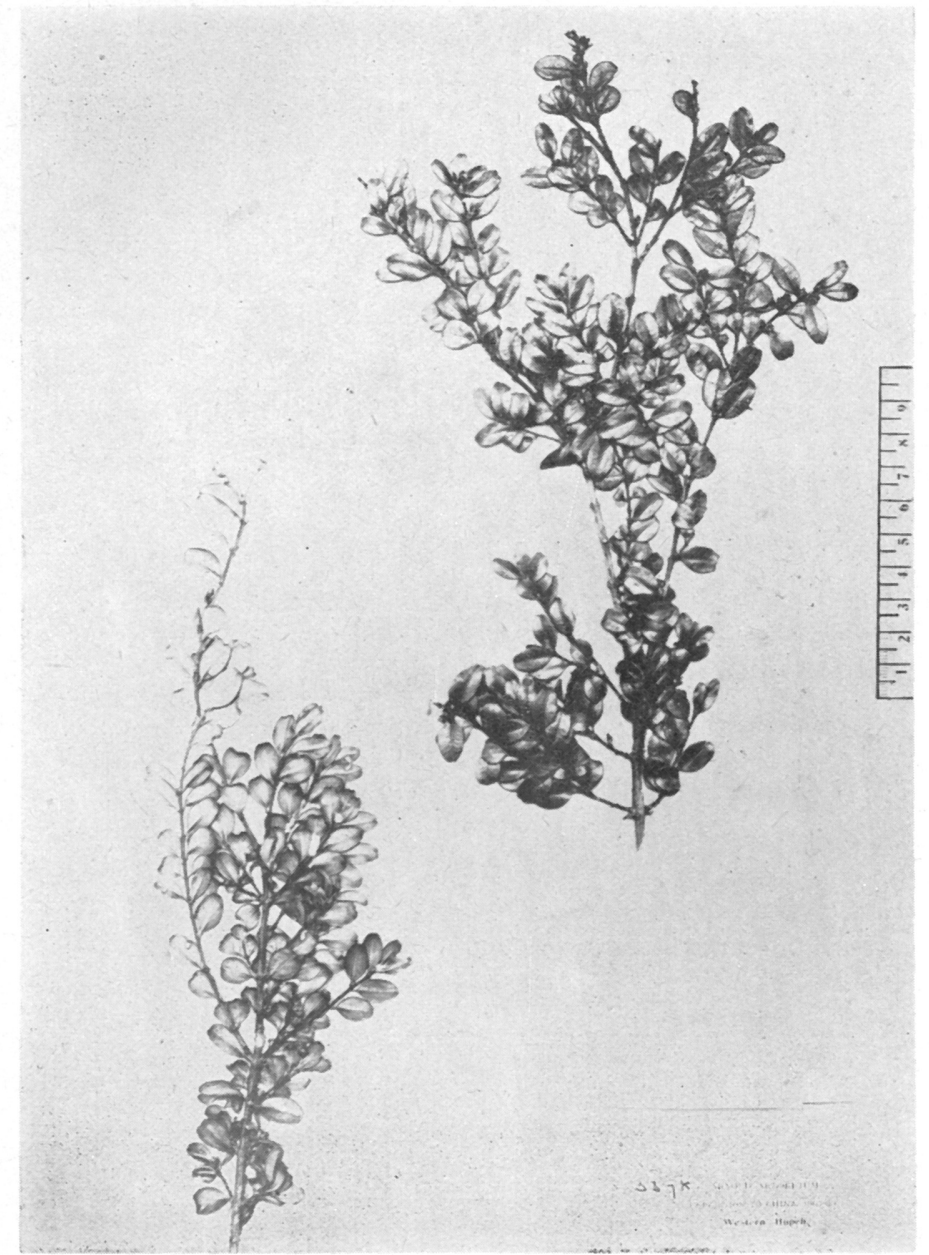

Fig. 1. Buxus microphylla subsp. sinica Hatusima (a smallleaved form from Hupeh; WiLson no. 3398).

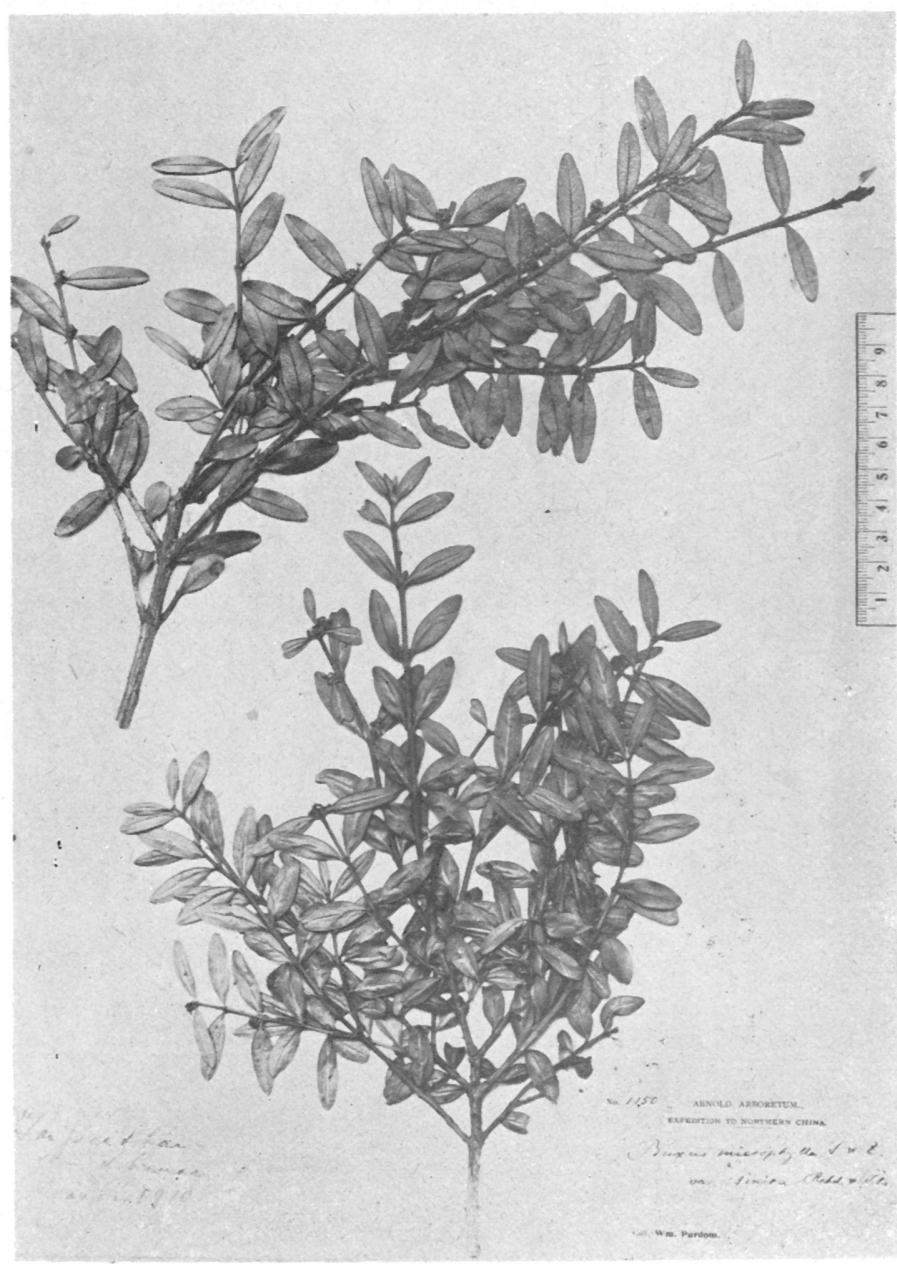

Fig. 2. Buxus micropnylla subsp. sinına Hatusima (PURDom, no.. 1150 from Shensi, with oblong leaves). 